\title{
Diversity in extinction laws of Type Ia supernovae measured between 0.2 and $2 \mu \mathrm{m}$
}

\author{
R. Amanullah, ${ }^{1 \star}$ J. Johansson, ${ }^{1}$ A. Goobar, ${ }^{1}$ R. Ferretti,${ }^{1}$ S. Papadogiannakis, ${ }^{1}$ \\ T. Petrushevska, ${ }^{1}$ P. J. Brown, ${ }^{2}$ Y. Cao,${ }^{3}$ C. Contreras, ${ }^{4}$ H. Dahle,${ }^{5}$ N. Elias-Rosa, ${ }^{6}$ \\ J. P. U. Fynbo, ${ }^{7}$ J. Gorosabel, ${ }^{8,9}$ L. Guaita, ${ }^{10}$ L. Hangard, ${ }^{1}$ D. A. Howell, ${ }^{11,12}$ \\ E. Y. Hsiao, ${ }^{13,4}$ E. Kankare ${ }^{14}$ M. Kasliwal, ${ }^{15}$ G. Leloudas, ${ }^{16,7}$ P. Lundqvist, ${ }^{17}$ \\ S. Mattila, ${ }^{18}$ P. Nugent, $,{ }^{19}, 20$ M. M. Phillips, ${ }^{3}$ A. Sandberg, ${ }^{17}$ V. Stanishev, ${ }^{21}$ \\ M. Sullivan, ${ }^{22}$ F. Taddia,,${ }^{17}$ G. Östlin, ${ }^{17}$ S. Asadi, ${ }^{17} \dagger$ R. Herrero-Illana,${ }^{8} \dagger$ \\ J. J. Jensen, ${ }^{7} \dagger$ K. Karhunen, ${ }^{23} \dagger$ S. Lazarevic, ${ }^{24} \dagger$ E. Varenius,${ }^{25} \dagger$ P. Santos,${ }^{5} \dagger$ \\ S. Seethapuram Sridhar, ${ }^{26,27} \dagger$ S. H. J. Wallström ${ }^{25} \dagger$ and J. Wiegert ${ }^{25} \dagger$
}

Affiliations are listed at the end of the paper

Accepted 2015 July 6. Received 2015 June 30; in original form 2015 April 8

\begin{abstract}
We present ultraviolet (UV) observations of six nearby Type Ia supernovae (SNe Ia) obtained with the Hubble Space Telescope, three of which were also observed in the near-IR (NIR) with Wide-Field Camera 3. UV observations with the Swift satellite, as well as ground-based optical and NIR data provide complementary information. The combined data set covers the wavelength range $0.2-2 \mu \mathrm{m}$. By also including archival data of SN $2014 \mathrm{~J}$, we analyse a sample spanning observed colour excesses up to $E(B-V)=1.4$ mag. We study the wavelength-dependent extinction of each individual $\mathrm{SN}$ and find a diversity of reddening laws when characterized by the total-to-selective extinction $R_{V}$. In particular, we note that for the two SNe with $E(B-V) \gtrsim 1 \mathrm{mag}$, for which the colour excess is dominated by dust extinction, we find $R_{V}=1.4 \pm 0.1$ and $R_{V}=2.8 \pm 0.1$. Adding UV photometry reduces the uncertainty of fitted $R_{V}$ by $\sim 50$ per cent allowing us to also measure $R_{V}$ of individual low-extinction objects which point to a similar diversity, currently not accounted for in the analyses when SNe Ia are used for studying the expansion history of the Universe.
\end{abstract}

Key words: circumstellar matter-supernovae: general-supernovae: individual: $\mathrm{SNe}$ 2012cg-supernovae: individual: 2012cu-dust, extinction.

\section{INTRODUCTION}

Studies of the cosmological expansion history using Type Ia supernovae (SNe Ia) have greatly improved our understanding of the Universe. While pioneering work lead to the discovery of the accelerated expansion from a few dozen SNe Ia (Riess et al. 1998; Perlmutter et al. 1999), the present samples of several hundred SNe Ia out to $z \sim 1.5$ show to high-precision that Einstein's Cosmological Constant provides an excellent fit for this phenomenon (e.g. Amanullah et al. 2010; Conley et al. 2011; Sullivan et al. 2011; Suzuki et al. 2012; Betoule et al. 2014).

^E-mail: rahman@fysik.su.se

$\dagger$ Participant of the Nordic Millimetre and Optical/NIR Astronomy Summer School 2012.
These findings have been possible due to the great accuracy of $\mathrm{SNe}$ Ia as distance indicators when observed in two or more broadband filters over several weeks, starting before light curve peak (see Goobar \& Leibundgut 2011, for a recent review). The distance to each individual supernovae ( $\mathrm{SN}$ ) is obtained by fitting an SN Ia template to the photometric data (see e.g. Riess, Press \& Kirshner 1996; Nugent, Kim \& Perlmutter 2002; Guy et al. 2007a; Jha, Riess, \& Kirshner 2007; Conley et al. 2008; Burns et al. 2011, and references therein) in order to obtain the brightness and colour at maximum together with the light-curve shape. The fitted properties are then typically combined using empirical linear relations to form a distance-dependent quantity (Phillips 1993; Tripp 1998).

The colour correction is based on the observation that red $\mathrm{SNe}$ are fainter than bluer ones, which could originate from a combination of an intrinsic SN Ia colour law, and extinction by dust in the host galaxy. The colour-brightness relation is commonly determined by minimizing the scatter around the best-fitting cosmological model, 
but there is no a priori reason why the two effects should follow the same colour-luminosity relation, nor can it be expected that host galaxy dust should have the same properties in different SN environments. In fact, even in the Milky Way, a range of extinction properties have been observed for different lines of sight.

When $\mathrm{SNe}$ Ia are used to measure distances, these uncertainties are handled by introducing a systematic error on the colourluminosity relation, which makes an important contribution to the error budget when propagated to the derived cosmological parameters. Addressing the colour-brightness relation, and in particular breaking the degeneracy between the intrinsic colour component and dust extinction, is important for future SN Ia surveys to improve beyond the current cosmological constraints.

The properties and wavelength-dependent extinction of dust in the Milky Way has been carefully studied and is commonly characterized by the total, $A_{V}$, to selective, $E(B-V)=A_{B}-A_{V}$, extinction ratio as $R_{V}=A_{V} /\left(A_{B}-A_{V}\right)$. Lower values of $R_{V}$ correspond to steeper extinction laws, since, for a given total extinction, $A_{V}$, these imply a larger reddening, $E(B-V)$.

The reddening of SNe Ia can be studied by comparing observed colours between reddened and similar un-reddened objects. Several such studies of individual SNe Ia (e.g. Elias-Rosa et al. 2006, 2008; Krisciunas et al. 2007; Folatelli et al. 2010; Amanullah et al. 2014, from hereon A14) in nearby galaxies, point to significantly lower values of $R_{V}$ than has been observed in the Milky Way. Since the reddening of several of these SNe Ia is significantly higher than what can be expected from the Milky Way extinction along the line of sight, the observed colour excesses are likely dominated by extinction in the $\mathrm{SN}$ host galaxies. For low-reddening $\mathrm{SNe}$ this may not be the case, but it is by nature difficult to measure $R_{V}$ to high precision for individual SNe with low $E(B-V)$. However, the global reddening law for a sample of $\mathrm{SNe}$ can be measured, and several studies have obtained low- $R_{V}$ values (e.g. Astier et al. 2006; Nobili \& Goobar 2008; Burns et al. 2014, from hereon B14) using this approach.

Chotard et al. (2011) and Scolnic et al. (2014) noted that globally derived reddening laws will be biased if the intrinsic SN Ia colour dispersions are not accounted for correctly, and Chotard et al. (2011) find $R_{V}=2.8 \pm 0.3$, consistent with the Milky Way average, for their sample of $76 \mathrm{SNe}$ Ia of which 73 had $E(B-V)<0.3$ mag. B14 also obtain $R_{V} \approx 3$ when they constrain their analysis to only include low-extinction $\mathrm{SNe}$ Ia, while highly reddened objects appear to prefer lower values $\left(R_{V} \approx 1.7\right)$ which is in agreement with previous studies (Folatelli et al. 2010). This suggests that a single, global, reddening law derived from a set of $\mathrm{SNe}$ Ia could depend on the colour distribution of the sample.

The degeneracy between intrinsic SNe Ia colour variations and dust extinction, can also be approached by studying the relation between SN colours and spectroscopic properties. Wang et al. (2009) found $R_{V}=1.6$ for SNe Ia with 'high' photospheric velocities (HV, $>11800 \mathrm{~km} \mathrm{~s}^{-1}$ ) within $5 \mathrm{~d}$ of maximum, while they obtained $R_{V}=2.4$ for objects with 'normal' velocity (NV, $<11800 \mathrm{~km} \mathrm{~s}^{-1}$ ). The expansion velocities were quantified by measuring the velocity of the Si II $6355 \AA$ absorption feature. Foley \& Kasen (2011) confirmed their findings but also conclude that when SNe with $E(B-$ $V)>0.35$ mag are omitted, they obtain $R_{V} \approx 2.5$ for the two separate subsamples, which also have different intrinsic colours. In other words, these findings also suggest that low- $R_{V}$ values are primarily associated with high-extinction, and these could dominate when a single global reddening law is derived from a sample of SNe Ia.

One natural explanation for low- $R_{V}$ values arises if $\mathrm{SNe}$ Ia are surrounded by circumstellar (CS) dust (Wang 2005; Goobar 2008).
In this scenario, photons can scatter back into the line of sight which reduces the total extinction. Photons that scatter on the CS dust will also arrive later than photons that do not interact which will give rise to time-dependent reddening (Wang 2005; Amanullah \& Goobar 2011; Brown et al. 2015). An observation will be a superposition of the SN spectrum at the given epoch and SN light from earlier epochs, but the scattering cross-section is wavelengthdependent so the fraction of delayed photons will also vary with wavelength. Further, depending on the geometry, scattered photons could give rise to a plateau for the late-time tail of the light curve and indications of such tails have been observed in $B-V$ (Förster et al. 2013).

CS dust could also be heated by the $\mathrm{SN}$, where the temperature will depend on the dust properties and the distance from the explosion. Heated dust has been detected in mid- to far-infrared for a subset of peculiar SNe Ia (Fox et al. 2011; Taddia et al. 2012; Fox \& Filippenko 2013; Silverman et al. 2013a), but Herschel and Spitzer observations (Johansson, Amanullah \& Goobar 2013; Johansson et al. 2014) of a handful of normal SNe Ia show no signs of heated CS dust (see also Maeda, Nozawa \& Motohara 2014, for limits from near-IR observations). For two of these SNe, 2006X and 2007le, together with SNe 1999cl and the SN 2002iclike PTF11kx, time-varying $\mathrm{Na}$ ID absorption features have been observed in their spectra on time-scales comparable to the SN lifetime which is consistent with the presence for CS material (Patat et al. 2007; Blondin et al. 2009; Simon et al. 2009; Dilday et al. 2012). Absorption from $\mathrm{Na}$ I has been used as a proxy for dust extinction (see e.g. Poznanski, Prochaska \& Bloom 2012), although high-resolution spectra are required (Poznanski et al. 2011) and its validity for extinction along lines-of-sight of SNe Ia has been challenged (Phillips et al. 2013). Time-varying Na ID can be explained by photo-ionization of neutral sodium (e.g. Borkowski, Blondin \& Reynolds 2009) and subsequent recombination by the SN. In a recent study by Soker (2014) this explanation has been questioned and an alternative model of photon-induced desorption of sodium from dust in planetary nebulae remnants was proposed. It has also been suggested that time-variations may originate from patchy interstellar material along the line of sight (Patat et al. 2010). More recently, Graham et al. (2015b) claimed a detection of a time-varying K I absorption feature in 2014J, while the corresponding Na ID feature remained unchanged, which they argue is consistent with the presence of CS material at a radius of $r_{\text {dust }} \sim 10^{19} \mathrm{~cm}$ due to the different ionization cross-sections.

An extensive study with multi-epoch high-resolution spectra of $14 \mathrm{SNe}$ (Sternberg et al. 2014) have failed to reveal further examples of time varying $\mathrm{Na}$ ID. Nevertheless, a statistically significant preponderance of $\mathrm{Na}$ ID features which are blue shifted with respect to the local velocity have been observed in high- and mid-resolution spectra (Sternberg et al. 2011; Maguire et al. 2013, respectively), suggesting that there is outflowing material from the $\mathrm{SN}$ progenitor system.

In this work, we seek to expand our knowledge of extragalactic extinction in the line of sight and SN Ia reddening by making the widest wavelength study of a sample of SNe Ia to date. We present UV-to-NIR light curves for six SNe Ia based on data mainly obtained from The Nordic Optical Telescope (NOT) and The Hubble Space Telescope (HST). The observations and data reduction is presented in Section 2 while the light curves and colours are presented in Section 3. The method for deriving individual reddening laws for each $\mathrm{SN}$ is described in Section 4, and after adding SN 2014J from A14 to the sample we present the results in Section 4.3. We search for CS dust in Section 5 and all the 
Table 1. SN coordinates are quoted from the discovery telegrams. All host galaxy data were obtained from the Nasa Extragalacic Database (NED), unless otherwise specified, where $v_{\mathrm{h}}$ is the measured recession velocity, and the Milky Way extinctions are from the Schlafly \& Finkbeiner (2011) calibration of the Schlegel, Finkbeiner \& Davis (1998) infrared-based dust maps. The projected distances from the host galaxy nuclei, $r_{\perp}$, were calculated based on the host galaxy distances and the SN offsets specified in Appendix A.

\begin{tabular}{lcccccccrr}
\hline SN & $\begin{array}{c}\alpha_{\mathrm{SN}} \\
(2000)\end{array}$ & $\begin{array}{c}\delta_{\mathrm{SN}} \\
(2000)\end{array}$ & $\begin{array}{c}r_{\perp} \\
(\mathrm{kpc})\end{array}$ & Host galaxy & $\begin{array}{c}\alpha_{\mathrm{h}} \\
(2000)\end{array}$ & $\begin{array}{c}\delta_{\mathrm{h}} \\
(2000)\end{array}$ & $\begin{array}{c}v_{\mathrm{h}} \\
\left(\mathrm{km} \mathrm{s}^{-1}\right)\end{array}$ & $\begin{array}{c}\text { Distance } \\
(\mathrm{Mpc})\end{array}$ & $\begin{array}{c}\text { MW } A_{V} \\
(\mathrm{mag})\end{array}$ \\
\hline $2012 \mathrm{bl}$ & $20: 23: 55.28$ & $-48: 21: 17.3$ & $15.6(0.2)$ & ESO 234-19 & $20: 23: 51.0$ & $-48: 21: 32$ & 5608 & $71(1)^{a}$ & 0.098 \\
$2012 \mathrm{bm}$ & $13: 05: 45.66$ & $+46: 27: 52.9$ & $7.5(0.3)$ & UGC 8189 & $13: 05: 46.6$ & $+46: 27: 42$ & 7436 & $103(8)^{b}$ & 0.033 \\
$2012 \mathrm{cg}$ & $12: 27: 12.83$ & $+09: 25: 13.2$ & $1.3(0.2)$ & NGC 4424 & $12: 27: 11.6$ & $+09: 25: 14$ & 437 & $15(2)^{c}$ & 0.057 \\
$2012 \mathrm{cp}$ & $13: 47: 01.79$ & $+33: 53: 35.0$ & $1.9(0.1)$ & UGC 8713 & $13: 47: 01.2$ & $+33: 53: 37$ & 4956 & $57(3)^{d}$ & 0.058 \\
$2012 \mathrm{cu}$ & $12: 53: 29.35$ & $+02: 09: 39.0$ & $5.4(1.2)$ & NGC 4772 & $12: 53: 29.1$ & $+02: 10: 06$ & 1040 & $41(9)^{e}$ & 0.074 \\
$2012 \mathrm{et}$ & $23: 42: 38.82$ & $+27: 05: 31.5$ & $2.7(0.2)$ & MCG +04-55-47 & $23: 42: 38.4$ & $+27: 05: 31$ & 7483 & $105(7)^{a}$ & 0.221 \\
2014J & $09: 55: 42.11$ & $+69: 40: 25.9$ & $1.0(0.3)$ & M82 & $09: 55: 52.7$ & $+69: 40: 46$ & 203 & $3.5(0.3)^{f}$ & $0.189^{f}$ \\
\hline
\end{tabular}

${ }^{a}$ Parnovsky \& Parnowski (2010); ${ }^{b}$ Calculated based on the redshift with $H_{0}=73 \pm 5 \mathrm{~km} \mathrm{~s}^{-1} / \mathrm{Mpc}$; ${ }^{c}$ Cortés, Kenney \& Hardy (2008);

${ }^{d}$ Springob et al. (2009); ${ }^{e}$ Tully et al. (2009); ${ }^{f}$ Dalcanton et al. (2009).

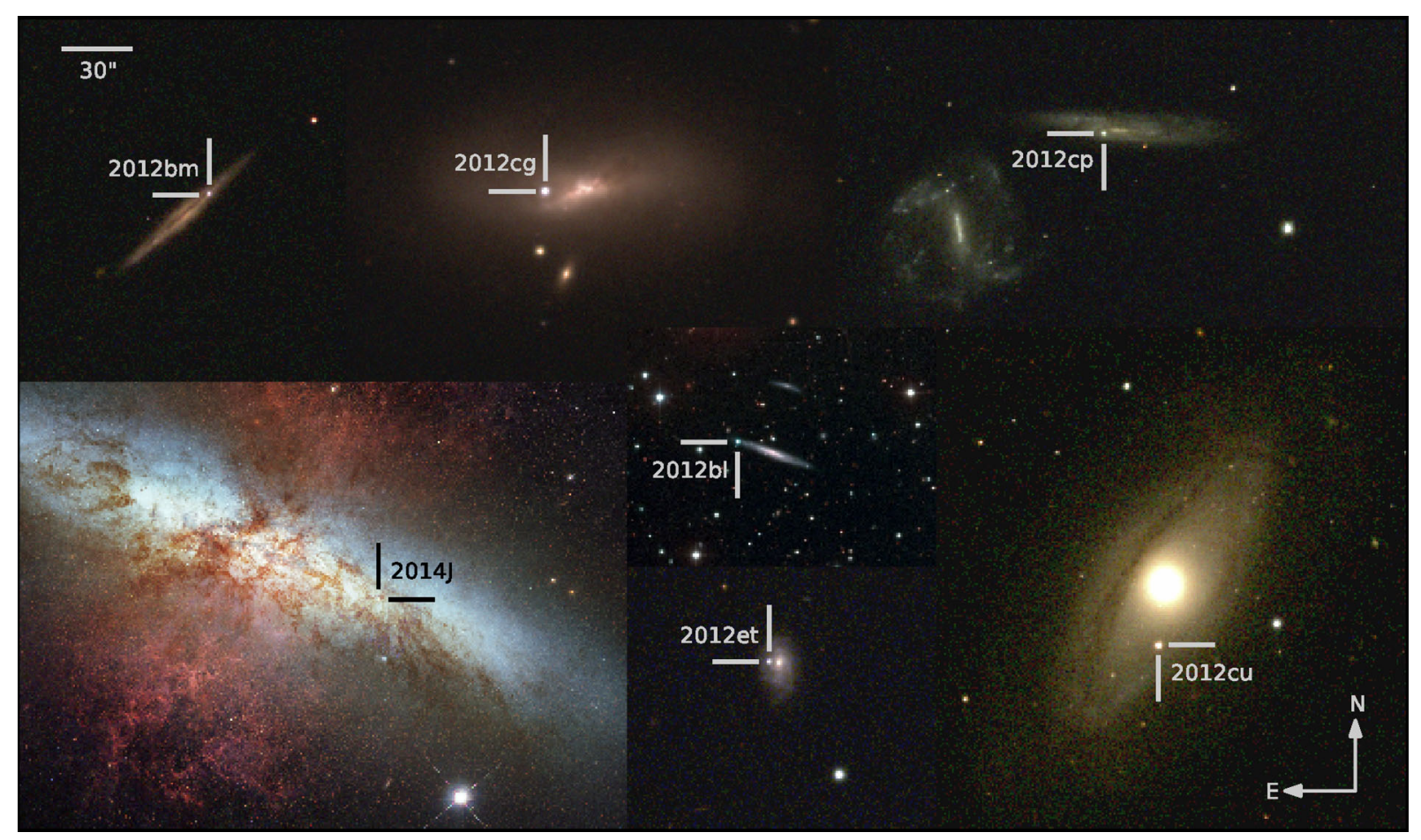

Figure 1. The seven SNe analysed in this work. The patches for SNe 2012bm, 2012cg, 2012cp, 2012cu and 2012et were obtained from BVR images from NOT, while the images for SNe 2012bl and 2014J are obtained from the Swope telescope and HST/WFC3 (Program DD-13621; PI Goobar) images, respectively.

results are discussed in Section 6. We conclude and summarize in Section 7.

All seven $\mathrm{SNe}^{1}$ are listed in Table 1 and shown together with their host galaxies in Fig. 1. They are also briefly summarized in Appendix A.

\section{OBSERVATIONS AND DATA REDUCTION}

\subsection{Hubble Space Telescope}

All previously unpublished $H S T$ observations discussed in this work are listed in Table 2. Each SN was observed at two different epochs (four for SN 2012et) with the Wide-Field Camera 3 (WFC3) during

\footnotetext{
${ }^{1}$ All data and figures presented in this work are available at http://snova.fysik.su.se/dust/
}

the HST Cycle 19 under programme GO-12582 (PI: Goobar). All SNe were imaged with the WFC3/UVIS channel through the passbands $F 225 W, F 275 W$ and $F 336 W$. In addition to this, NIR imaging in the $H S T$ passbands $F 125 W$ and $F 160 W$ was obtained for the $\mathrm{SNe}$ 2012cg, 2012cu and 2012et with the WFC3/IR channel. Examples of both the WFC3/UVIS and WFC3/IR observations are shown in Fig. 2.

WFC3/UVIS consists of two $4096 \times 2051$ e2V CCD detectors with a plate scale of $0.04 \mathrm{arcsec}_{\text {pixel }}^{-1}$, while the Teledyne $\mathrm{HgCdTe}$ infrared detector used in the WFC3/IR channel has a pixel scale of 0.13 \%/pixel. For each observation, only the sub-arrays (apertures), listed in Table 2, of the detectors were read-out. All WFC3 data were reduced using the standard STScI reduction pipeline and calibrated using CALWF3.

The WFC3/UVIS CCDs, like all HST CCDs, are plagued with charge transfer inefficiencies (CTI), i.e. degradations of the detector 
Table 2. Wide-Field Camera-3 and STIS observations together with the used apertures, where UVIS1 and UVIS2 are the two chips of the WFC3/UVIS channel. The observations with the WFC3/IR and WFC3/UVIS channels were obtained through the $F 125 \mathrm{~W}, F 160 \mathrm{~W}$ and $F 225 \mathrm{~W}, F 275 \mathrm{~W}$, $F 336 W$ broad-band passbands, respectively.

\begin{tabular}{lcll}
\hline Civil date & \multirow{2}{*}{ MJD } & \multicolumn{1}{c}{ Aperture } & SN \\
\hline $2012-04-09$ & 56026.4 & UVIS1-2K2A-SUB & $2012 \mathrm{bm}$ \\
$2012-04-09$ & 56026.5 & STIS 52X0.2 G230LB & $2012 \mathrm{bm}$ \\
$2012-04-13$ & 56030.6 & UVIS1-2K2A-SUB & $2012 \mathrm{bm}$ \\
$2012-04-13$ & 56030.7 & STIS 52X0.2 G230LB & $2012 \mathrm{bm}$ \\
$2012-04-16$ & 56033.1 & STIS 52X0.2 G230LB & $2012 \mathrm{bl}$ \\
$2012-04-16$ & 56038.2 & UVIS1-2K2A-SUB & $2012 \mathrm{bl}$ \\
$2012-04-20$ & 56037.4 & STIS 52X0.2 G230LB & $2012 \mathrm{bl}$ \\
$2012-04-21$ & 56038.2 & UVIS1-2K2A-SUB & $2012 \mathrm{bl}$ \\
$2012-06-04$ & 56082.4 & UVIS2-M1K1C-SUB & $2012 \mathrm{cg}$ \\
$2012-06-04$ & 56082.4 & IRSUB64 & $2012 \mathrm{cg}$ \\
$2012-06-04$ & 56082.5 & STIS 52X0.2 G230LB & $2012 \mathrm{cg}$ \\
$2012-06-04$ & 56082.5 & STIS 52X0.1 G430L & $2012 \mathrm{cg}$ \\
$2012-06-18$ & 56096.4 & STIS 52X0.2 G230LB & $2012 \mathrm{cg}$ \\
$2012-06-18$ & 56096.4 & STIS 52X0.1 G430L & $2012 \mathrm{cg}$ \\
$2012-06-19$ & 56097.5 & UVIS2-M1K1C-SUB & $2012 \mathrm{cg}$ \\
$2012-06-19$ & 56097.5 & IRSUB64 & $2012 \mathrm{cg}$ \\
$2012-06-04$ & 56082.6 & UVIS1-2K2A-SUB & $2012 \mathrm{cp}$ \\
$2012-06-04$ & 56082.7 & STIS 52X0.2 G230LB & $2012 \mathrm{cp}$ \\
$2012-06-16$ & 56094.6 & UVIS1-2K2A-SUB & $2012 \mathrm{cp}$ \\
$2012-06-17$ & 56095.6 & STIS 52X0.2 G230LB & $2012 \mathrm{cp}$ \\
$2012-07-02$ & 56110.8 & UVIS2-M1K1C-SUB & $2012 \mathrm{cu}$ \\
$2012-07-02$ & 56110.8 & IRSUB512 & $2012 \mathrm{cu}$ \\
$2012-07-07$ & 56115.8 & UVIS2-M1K1C-SUB & $2012 \mathrm{cu}$ \\
$2012-07-07$ & 56115.8 & IRSUB512 & $2012 \mathrm{cu}$ \\
$2012-10-01$ & 56201.5 & UVIS2-M1K1C-SUB & $2012 \mathrm{et}$ \\
$2012-10-01$ & 56201.5 & IR & $2012 \mathrm{et}$ \\
$2012-10-01$ & 56201.8 & STIS 52X0.2 G230LB & $2012 \mathrm{et}$ \\
$2012-10-01$ & 56201.8 & STIS 52X0.2 G430L & $2012 \mathrm{et}$ \\
$2012-10-05$ & 56205.6 & UVIS2-M1K1C-SUB & $2012 \mathrm{et}$ \\
$2012-10-05$ & 56205.6 & IR & $2012 \mathrm{et}$ \\
$2012-10-09$ & 56209.8 & UVIS2-M1K1C-SUB & $2012 \mathrm{et}$ \\
$2012-10-09$ & 56209.8 & IR & $2012 \mathrm{et}$ \\
$2012-10-13$ & 56213.9 & UVIS2-M1K1C-SUB & $2012 \mathrm{et}$ \\
$2012-10-13$ & 56213.9 & IR & $2012 \mathrm{et}$ \\
\hline & & & \\
\hline
\end{tabular}

performance over time due to damage in the silicon lattice from cosmic rays. The CTI can be reverse corrected at the pixel level using wf c3uv_ctereverse ${ }^{2}$ for all frames except for images obtained with the UVIS2-M1K1C-SUB sub-array. For this aperture, we instead used the recipe suggested by Bourque \& Anderson (2014). The individual flat-fielded WFC3/UVIS and WFC3/IR images were then resampled and combined using astrodrizzle (Fruchter et al. 2010).

Aperture photometry was carried out on the combined images using an aperture radius of $0.4 \mathrm{arcsec}$, although in some cases a smaller aperture of radius 0.16 arcsec had to be used for the WFC3/UVIS data. This was the case for the low signal-to-noise measurements of SN 2012bm, observations of SN 2012cg that were plagued by nearby coincidence cosmic rays, and all epochs of SN 2012et, where a nearby object contaminated the larger aperture (see the mid-right panel of Fig. 2). The smaller radius measurements had to be aperture corrected to the 0.4 arcsec radius for which STScI provides
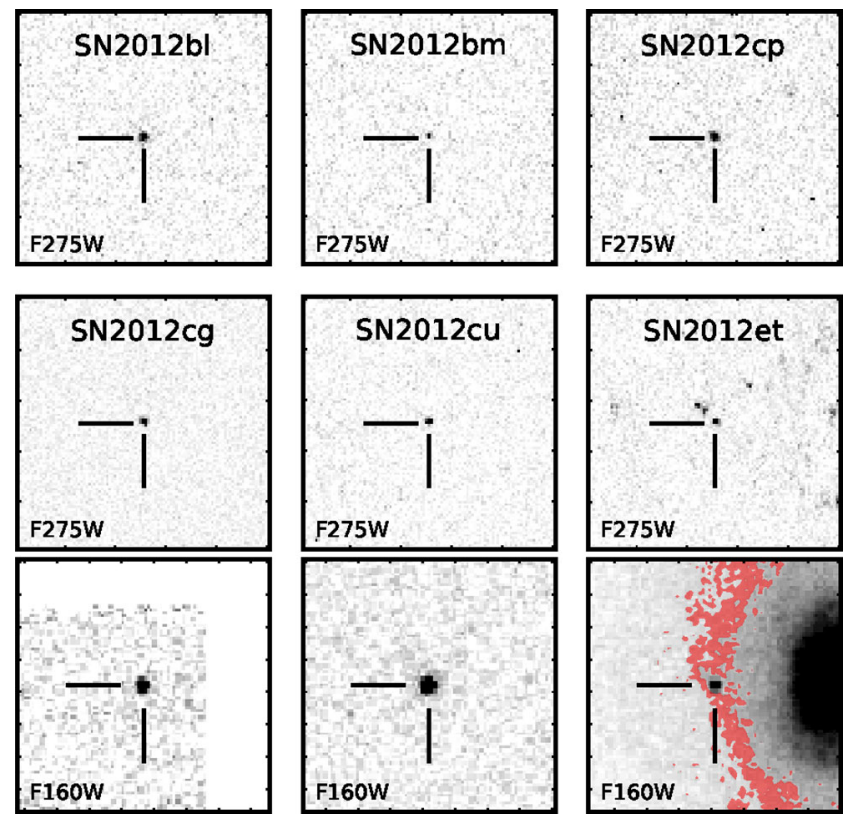

Figure 2. HST/WFC3 patches of the six SNe observed during Cycle 19. The patch sizes are $10 \operatorname{arcsec} \times 10 \operatorname{arcsec}$ and in the upper row the first epochs obtained through the $F 275 \mathrm{~W}$ filter is shown for each of the three SNe 2012bl, 2012bm and 2012cp. In the two lower rows, the corresponding patches are shown for the SNe 2012cg, 2012cu and 2012et, together with the NIR observations in the $F 160 \mathrm{~W}$ filter that also were obtained for these objects. The marked (red) region in the lower right panel show the isophot from which the host galaxy background of SN 2012et was estimated as explained in the text.

zero-points ${ }^{3}$ for WFC3. Since there were no bright stars present in the fields, we used the general enclosed energy tables provided by STScI for this purpose. To account for the time and wavelength dependence of the WFC3/UVIS aperture corrections, we adopt an additional uncertainty of 0.05 mag for all measurements using the 4 pixel radius, which was derived by comparing the enclosed energy tables with photometry from different aperture radii of the high signal-to-noise data.

Only the SN 2012et NIR measurements suffered from host galaxy contamination. The host contribution was estimated by placing apertures of the same radius used for the SN photometry along the isophot of the galaxy that intersects with the position of SN 2012et as illustrated in the lower right panel of Fig. 2. The background was then estimated as the median of these measurements, and the rootmean-square (RMS) of the measurements was added in quadrature to the photometric uncertainty. All the WFC3 photometry is presented in Table 3 and shown in Fig. 3. Here, we also present updated photometry of SN 2014J using the method described here which is consistent with the results presented in A14.

For most HST visits, we also obtained long-slit spectroscopy with the Space Telescope Imaging Spectrograph (STIS) where the $1024 \times 1024$ pixel SITe CCD detector was used with the G230LB grating covering the wavelength range 1600-3100 $\AA$, and for two SNe (2012cg, 2012et) G430L, 2900-5700 A. The data were reduced using the calstis pipeline which is part of the STSDAS package. The pipeline was ran for all the spectra up to the point where the calibrated 2D spectra were created. Only the spectra of SN 2012cg and the red G430L spectrum of SN 2012et contained any significant

\footnotetext{
${ }^{2}$ http://www.stsci.edu/hst/wfc3/tools/cte_tools
}

${ }^{3}$ We used the zero-points from 2012 March 06. 
Table 3. The photometry of all SNe. All magnitudes are in the natural Vega system. The rest-frame magnitude and colour can be obtained from Columns 4 to 6 using equation (1). Columns 5 and 9 are the Galactic extinctions and Columns 6 and 10 are $K_{X}$ and $K_{V}$ corrections for the two bands, respectively. All corrections were calculated after the SN 2011fe template had been reddened with the best fitted F99 law, shown in Table 5, for each SN. The $V$ magnitude is only shown for data points used in the colour analysis, i.e. with phases between -10 and +35 d. Column 2 show the effective light-curve-width-corrected phase, while Column 7 specifies whether the $V$ magnitude was measured for the same epoch (D) or if it was calculated using the sNoopy model (M). The corresponding intrinsic colour for the SN 2011fe template is shown when available in Column 11. The Swift magnitudes of SN 2014J are from Brown et al. (2015). (This table is available in its entirety in a machine-readable form in the online journal. A portion is shown here for guidance regarding its form and content.)

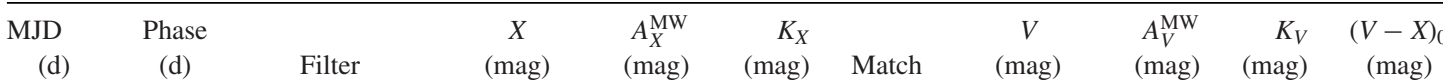

\begin{tabular}{rrllllllllr}
\hline \multicolumn{10}{c}{ SN2012cg } \\
56082.4 & 0.2 & WFC3 $F 336 W$ & $11.74(0.06)$ & 0.09 & 0.01 & M & $12.04(0.04)$ & 0.06 & 0.00 & 0.61 \\
56097.5 & 14.0 & WFC3 $F 336 W$ & $13.75(0.01)$ & 0.09 & 0.02 & M & $12.53(0.07)$ & 0.06 & 0.00 & -0.90 \\
56082.4 & 0.2 & WFC3 $F 125 W$ & $12.37(0.01)$ & 0.01 & 0.05 & M & $12.04(0.04)$ & 0.06 & 0.00 & -0.55 \\
56097.5 & 14.1 & WFC3 $F 125 W$ & $13.88(0.03)$ & 0.02 & -0.07 & M & $12.53(0.07)$ & 0.06 & 0.00 & -1.91 \\
56082.4 & 0.2 & WFC3 F225W & $16.38(0.05)$ & 0.13 & 0.02 & M & $12.04(0.04)$ & 0.06 & 0.00 & -2.55 \\
56097.5 & 14.0 & WFC3 F225W & $17.60(0.08)$ & 0.13 & 0.02 & M & $12.53(0.07)$ & 0.06 & 0.00 & -3.68 \\
56082.4 & 0.2 & WFC3 F160W & $12.67(0.02)$ & 0.01 & 0.01 & M & $12.04(0.04)$ & 0.06 & 0.00 & -0.88 \\
56097.5 & 14.1 & WFC3 F160W & $13.24(0.03)$ & 0.01 & 0.55 & M & $12.53(0.07)$ & 0.06 & 0.00 & -0.69
\end{tabular}
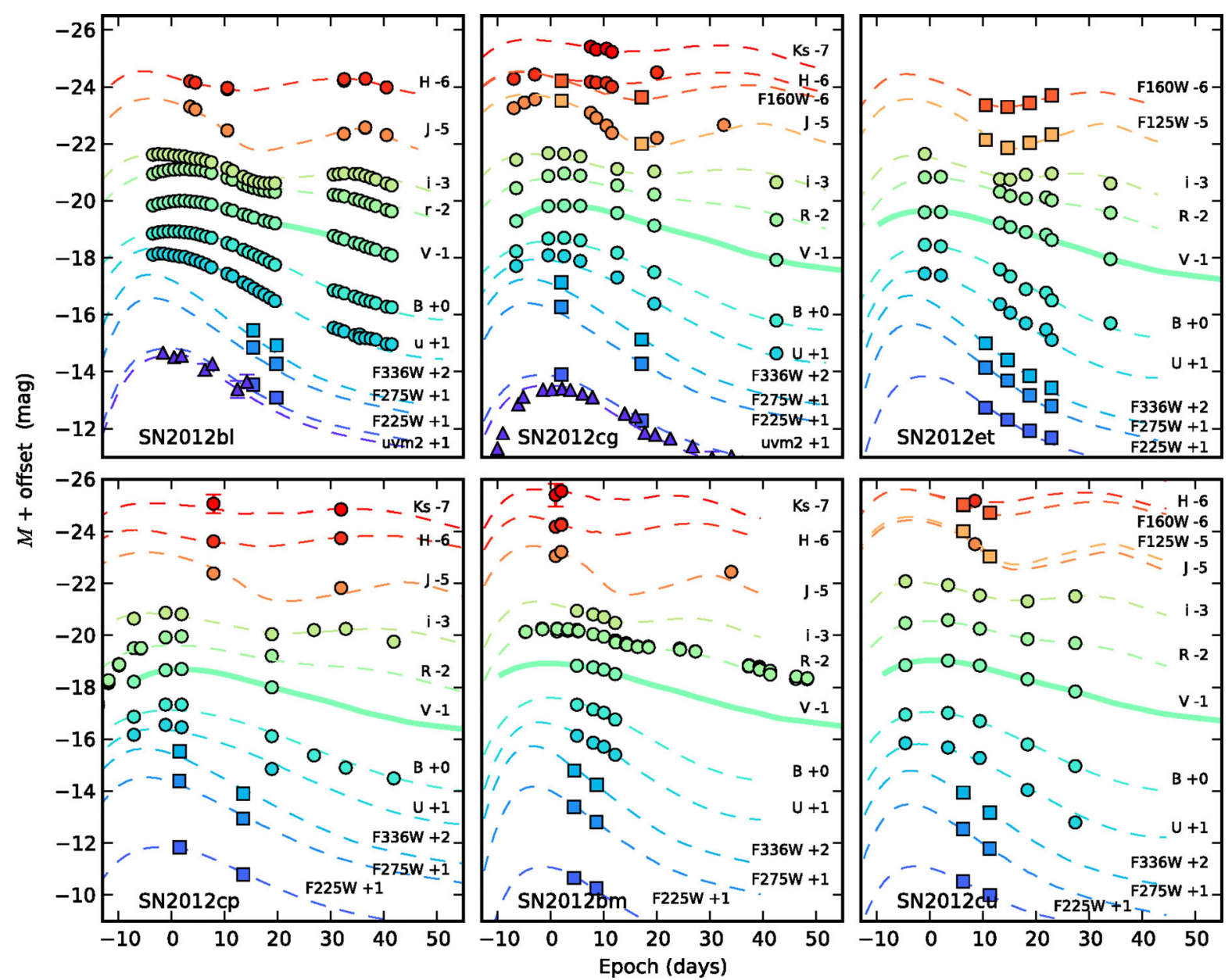

Figure 3. Observed light curves for the SN sample. Our analysis is based on optical UBVRI and NIR JHK photometry from ground-based observatories (circles), UV ( $F 225 W, F 275 W, F 336 W$ ) and NIR ( $F 125 W, F 160 W$ ) photometry from the $H S T$ (squares). For the SNe $2012 \mathrm{bl}$ and $2012 \mathrm{cg}$ we also use Swift/UVOT photometry (triangles). The data are shown in their instrumental system but have been shifted using the distance moduli based on the distances in Table 1 and offset for clarity. The thick, solid, lines show the sNOOPY $V$-models used to calculate colours as described in the text, while the dashed lines show synthesized light curves of SN 2011fe to guide the eye. When an SN has observations in a similar filter from multiple instruments only one of the light curves is shown. The epoch is the observed date with respect to the fitted day of maximum in the $B$ band from Table 5 . 
Table 4. Ground-based spectroscopic observations. The Asiago spectra were obtained through the Asiago Transient Classification Program (Tomasella et al. 2014). The epochs are shown with respect to the date of $B$-maximum shown in Table 5.

\begin{tabular}{lrrlrrr}
\hline Civil date & MJD & $\begin{array}{r}\text { Epoch } \\
(\mathrm{d})\end{array}$ & \multicolumn{1}{c}{ Instrument } & $\begin{array}{c}\lambda \\
(\AA)\end{array}$ & $\begin{array}{c}\text { Exp. } \\
(\mathrm{s})\end{array}$ & SN \\
\hline $2012-05-26$ & 56073.8 & +260 & WHT/ISIS & $3500-9500$ & 600 & $2011 \mathrm{fe}$ \\
$2012-03-30$ & 56016.3 & -2 & DuPont/WFCCD/WF4K-1 & $3600-9200$ & 700 & $2012 \mathrm{bl}$ \\
$2012-03-31$ & 56017.4 & -1 & DuPont/WFCCD/WF4K-1 & $3600-9200$ & 700 & $2012 \mathrm{bl}$ \\
$2012-05-01$ & 56048.3 & +29 & DuPont/WFCCD/WF4K-1 & $3600-9200$ & 900 & $2012 \mathrm{bl}$ \\
$2012-03-28$ & 56014.0 & -4 & ASIAGO/AFOSC & $3500-8200$ & 2700 & $2012 \mathrm{bm}$ \\
$2012-04-09$ & 56027.0 & +9 & NOT/ALFOSC & $3200-9000$ & 2400 & $2012 \mathrm{bm}$ \\
$2012-04-16$ & 56034.1 & +16 & NOT/ALFOSC & $3200-9000$ & 2400 & $2012 \mathrm{bm}$ \\
$2012-05-24$ & 56071.9 & -9 & NOT/ALFOSC & $3700-7200$ & 5477 & $2012 \mathrm{cg}$ \\
$2012-05-26$ & 56073.9 & -7 & NOT/FIES & $3640-7360$ & 1800 & $2012 \mathrm{cg}$ \\
$2012-05-28$ & 56075.9 & -5 & NOT/ALFOSC & $3700-7200$ & 6588 & $2012 \mathrm{cg}$ \\
$2012-06-02$ & 56080.9 & +1 & NOT/FIES & $3640-7360$ & 900 & $2012 \mathrm{cg}$ \\
$2012-06-04$ & 56082.9 & +3 & NOT/ALFOSC & $3200-9000$ & 300 & $2012 \mathrm{cg}$ \\
$2012-06-14$ & 56092.9 & +13 & NOT/FIES & $3640-7360$ & 1200 & $2012 \mathrm{cg}$ \\
$2013-03-09$ & 56360.4 & +280 & Keck/LRIS & $3075-10300$ & 600 & $2012 \mathrm{cg}$ \\
$2012-05-26$ & 56074.0 & -7 & NOT/ALFOSC & $3200-9000$ & 2700 & $2012 \mathrm{cp}$ \\
$2012-06-04$ & 56083.0 & +2 & NOT/ALFOSC & $3200-9000$ & 1800 & $2012 \mathrm{cp}$ \\
$2012-07-05$ & 56113.9 & +9 & NOT/ALFOSC & $3200-9000$ & 1200 & $2012 \mathrm{cu}$ \\
$2012-09-13$ & 56183.8 & -7 & ASIAGO/AFOSC & $3500-8200$ & 2700 & $2012 \mathrm{et}$ \\
$2012-09-22$ & 56193.1 & +2 & NOT/ALFOSC & $3200-9000$ & 600 & $2012 \mathrm{et}$ \\
$2014-11-09$ & 56970.0 & +282 & APO/DIS & $3300-9800$ & 750 & $2014 \mathrm{~J}$ \\
\hline
\end{tabular}

signal and were extracted using the calstis $\mathrm{x} 1 \mathrm{~d}$ routine with a 4 pixel aperture.

\subsection{Swift/UVOT}

UV photometry was also obtained with the Ultra-Violet/Optical Telescope (UVOT; Roming et al. 2005) on the Swift spacecraft (Gehrels et al. 2004) for the SNe 2012bl, 2012cg, 2012cp and $2012 \mathrm{cu}$ in the $u v w 1, u v w 2$ and $u v m 2$ filters. However, the $u v w 1$ and $u v w 2$ filters are not well suited for extinction studies due to the significant 'red-tails' of these filters. Milne et al. (2010) used the UV spectra of SN 1992A (Kirshner et al. 1993) and estimated that 52 per cent and 44 per cent of the light in $u v w 1$ and $u v w 2$, respectively, originate from wavelengths redder than $3000 \AA$. Brown et al. (2015) show how the observed photons and the corresponding effective wavelengths shift dramatically as reddening increases.

The $u v m 2$ filter, on the other hand, is much better constrained ( 1 per cent of the light comes from $>3000 \AA$ ) and is used for the extinction studies in this work. The $u v m 2$ magnitudes were measured using the pipeline from the Swift Optical/Ultraviolet Supernova Archive (SOUSA; Brown et al. 2014), including correction for the time-dependent sensitivity and revised zero-points from Breeveld et al. (2011). The result is presented in Table 3 and shown in Fig. 3. Only the SNe 2012bl and 2012cg showed a significant SN flux in the uvm 2 band after subtraction of the underlying host galaxy flux. The flux measured for SN 2012cp was consistent with the host galaxy brightness and no signal was measured for SN 2012cu.

\subsection{Ground-based observations}

The ground-based spectroscopic observations are listed in Table 4 while the photometric observations are summarized together with the measured magnitudes in Table 3 and shown in Fig. 3. All SNe except for SN 2012bl were observed with the 2.56 metre NOT under programmes 45-009 and 46-018 (PI: Amanullah). Imaging and spectroscopy was obtained with the $6.4 \mathrm{arcmin} \times 6.4 \mathrm{arcmin}$ Andalucia Faint Object Spectrograph and Camera (ALFOSC) using the filter set $U(\# 7),{ }^{4} B(\# 74), V(\# 75), R(\# 76), i(\# 12)$ and the $R=360$ grism (\#4). ${ }^{5}$ The data were reduced using standard IRAF routines and the QUBA pipeline (Valenti et al. 2011). The photometry was measured by first fitting the point spread function (PSF) using DAOPHOT (Stetson 1987) to stars in the fields. The calibrated magnitudes were obtained either from Landolt fields (Landolt 1992) observed during the night, or, when these were not available, by comparing the fluxes to stars in the fields that were then calibrated against Landolt fields for the photometric nights.

With the NOT, we also obtained high-resolution spectroscopy of SN 2012cg using the FIbre-fed Echelle Spectrograph (FIES; Telting et al. 2014) in its high-resolution mode, $R=67000$. Simultaneous wavelength reference (Thorium-Argon) spectra were obtained and the data were reduced using the software FIESTOOL which is provided by the observatory.

NIR observations were carried out for SNe 2012bm, 2012cg and $2012 \mathrm{cp}$ with the $4 \operatorname{arcmin} \times 4 \operatorname{arcmin}$ NOTCam instrument in the $J$, $H$ and $K s$ bands under the programmes 45-007 (PI: Kankare) and 46-020 (PI: Mattila). We used the wide field imaging option of the $1024 \times 1024$ pixel $\mathrm{HgCdTe}$ NOTCam detector with a plate scale of 0.234 arcsec pixel $^{-1}$. The observations were carried out using either 5 or 9-point dithering patterns. For the $\mathrm{SNe}$ with extended host galaxies, beam-switching was used to guarantee a successful sky subtraction. We used the NOTCam Quick-Look reduction package based on IRAF. Bad pixels (which includes two dead columns) were

\footnotetext{
${ }^{4}$ NOT filter ID.

${ }^{5}$ NOT grism ID.
} 
masked and we used master differential skyflats which were obtain from bright and faint skyflats. Further, the images were corrected for geometric distortions ${ }^{6}$ before the individual images were aligned and co-added.

SN 2012cg was also observed in JHKs with the $4.3 \operatorname{arcmin} \times 4.3$ arcmin CAIN instrument on the 1.52 metre Carlos Sanchez Telescope at Observatorio del Teide on Tenerife. CAIN III is a $256 \times 256$ pixel $\mathrm{HgCdTe}$ NIR detector, where we used the wide field option with a plate scale of 1.0 arcsec pixel ${ }^{-1}$. The data were reduced using a dedicated IRAF package provided by J. Pullido and A. Barrena.

The two SNe 2012bm and 2012cp were also observed by the Palomar Transient Factory with the Palomar 48-inch telescope (Rau et al. 2009) in the Mould $R$. We use their photometry which was obtained by first determining the PSF prior to subtractions, and then carrying out PSF photometry on the subtracted frames (Firth et al. 2015). The calibration of this data has been described in Ofek et al. (2012)

Photometric follow-up of SN 2012bl was carried out by the Carnegie Supernova Project using the Swope telescope for the optical and the du Pont telescope for the NIR. The reduction and photometry have been described in detail in Hamuy et al. (2006) and Contreras et al. (2010). PSF photometry was performed with respect to a local sequence of standard stars calibrated to the Landolt (1992) and Smith et al. (2002) standard fields for the optical observations and the Persson et al. (1998) for the NIR.

Further, we here also present spectroscopic observations of $\mathrm{SNe}$ $2011 \mathrm{fe}, 2012 \mathrm{cg}$ and $2014 \mathrm{~J}$ obtained using the Intermediate dispersion Spectrograph and Imaging System (ISIS) instrument at the William Herschel Telescope, the Low Resolution Imaging Spectrometer (LRIS) instrument at Keck and the Dual Imaging Spectrograph (DIS) instrument at the Apache Point Observatory. These data were reduced in a standard manner.

\section{SN SPECTRA AND COLOURS}

The spectra for the seven $\mathrm{SNe}$ around maximum are shown in Fig. 4, where we also added the classification spectrum of SNe 2012bm and 2012et (Tomasella et al. 2014) to the set of spectroscopic observations already described above. For SN 2012cg, both the HST/STIS G250LB and G430L spectra, and the ALFOSC spectrum, that was obtained the same day, are shown. We used SNID (Blondin \& Tonry 2007) to type the spectra and all SNe could be classified as 'normal', although SN 2012et also provided acceptable matches to SN 1999aa. For each spectrum in Fig. 4, we also show the spectrum of the normal SN 2011fe for the matching epoch (Mazzali et al. 2014) for comparison.

All photometric data are shown in Fig. 3 and for each measurement in each filter, $X$, where $X \in\{u v m 2, F 225 W, \ldots, K s\}$, we calculated the colour $X-V$. When $X$ and $V$-band measurements are not available for the same dates, and the colour could not be obtained directly, a $V$-band model was used to calculate the colours. When applicable, we used a smoothed spline model but for the $\mathrm{SNe}$ 2012 bm and 2012cp the $V$-band data were too sparsely sampled to allow reliable spline fits. For these objects we instead fitted the SN Ia template from Hsiao et al. (2007, H07 from hereon) to the observed data. Both the spline and template-based models were obtained using the SNOOPY light-curve fitter (Burns et al. 2011) and are shown as thick lines in Fig. 3. Further, for all model-based colours

\footnotetext{
${ }^{6}$ Gålfalk, (private communication).
}

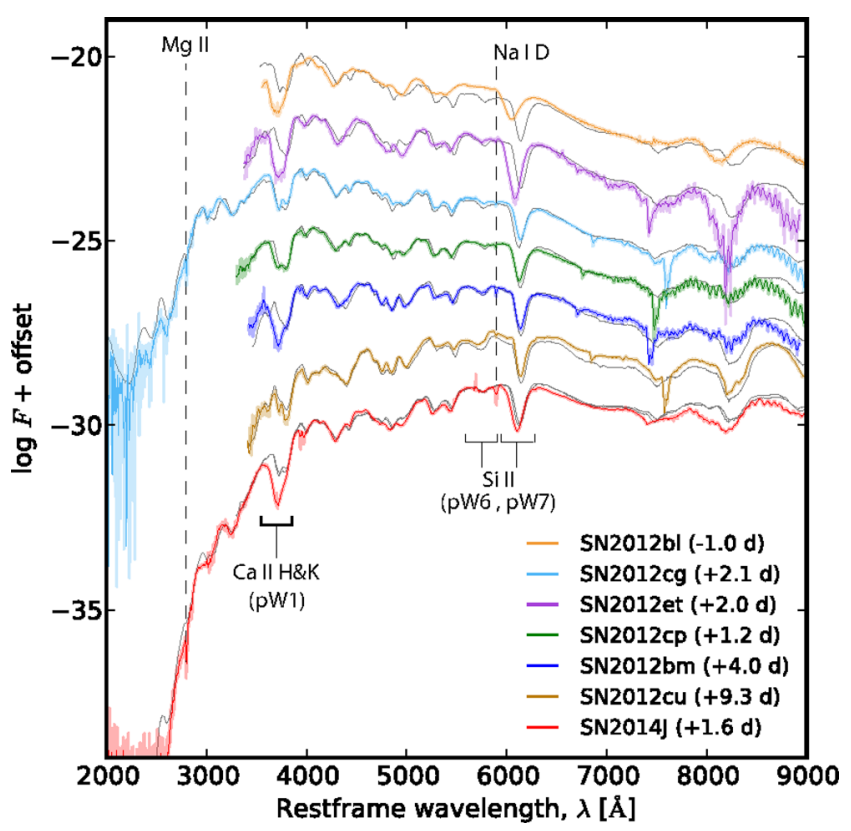

Figure 4. Spectra near maximum, when available, for the SNe in our sample. The grey spectra are for SN 2011fe at similar epochs (Pereira et al. 2013; Mazzali et al. 2014) reddened by the extinction laws that we derive in Section 4. For SNe 2012cg and 2014J, we have combined the optical spectra with STIS spectra (the SN 2014J spectrum was taken from Foley et al. 2014). Spectroscopic features discussed in Sections 5, 6.1 and 6.4 have been marked.

we add 0.05 mag in quadrature to uncertainties in order to account for the inaccuracy of the models.

All colours were corrected for Galactic extinction, $A^{\mathrm{MW}}$, using the extinction law by Cardelli, Clayton \& Mathis (1989, CCM from hereon) with $R_{V}=3.1$ and the $A_{V}^{\mathrm{MW}}$ values shown in the last column of Table 1 . The values are from the Schlafly \& Finkbeiner (2011) recalibration of the Schlegel et al. (1998) dust maps, except for SN 2014J where we use a value obtained from studying neighbouring regions (Dalcanton et al. 2009).

The colours were also $K$-corrected (e.g. Nugent et al. 2002) and $S$-corrected (e.g. Suntzeff 2000; Stritzinger et al. 2002; Krisciunas et al. 2003) to a common rest-frame filter system that will be used for the remaining analysis throughout the paper. The analysis was carried out using the WFC3/UVIS and Swift/UVOT filters for the UV, the ALFOSC filter set for the optical and the NOTCam filters for the NIR, since the bulk of the data were obtained using these filters. The combined $K$ - and $S$-corrections ( $K_{X}$ from now on) are calculated synthetically using the spectral energy distribution (SED) of SN $2011 \mathrm{fe}$ as described in Section 4.1 and the filter transmissions provided by the different observatories.

To summarize, all colours were obtained as

$X-V=\left(m_{X}-A_{X}^{\mathrm{MW}}-K_{X}\right)-\left(\mathcal{M}_{V}-A_{V}^{\mathrm{MW}}-K_{V}\right)$,

where $m_{X}$ are the measured instrumental magnitudes in filter $X$ and $\mathcal{M}_{V}$ is the $V$ magnitude for the same date either measured directly, or obtained from a spline or template model. All values are presented in Table 3 where we have also added the Swift photometry of SN 2014J from Brown et al. (2015) to the measurements described in Section 2. As will be further discussed in Section 4, not only the observed colours, but also, $A^{\mathrm{MW}}$ and $K_{X}$ depend on the reddening of each SN and were therefore obtained iteratively during the extinction law fitting. The values shown in Table 3 were obtained after the SN 2011fe SED had been reddened with the Fitzpatrick 
Table 5. The best fitted parameters to all measured colours between phases -10 and $+35 \mathrm{~d}$ for the reddening laws investigated in this work. For the power law, we have fixed $a$ to $a \equiv 1$ to break the degeneracy between this parameter and $A_{V}$. For each value, we quote the 68 per cent level fitted uncertainty when the parameters are considered individually. The degrees of freedoms were calculated under assumptions that the colours are independent measurements, which is not the case.

\begin{tabular}{|c|c|c|c|c|c|c|c|}
\hline & & $2012 \mathrm{cg}$ & 2012et & $2012 \mathrm{cp}$ & $2012 \mathrm{bm}$ & $2012 \mathrm{cu}$ & $2014 J$ \\
\hline & $R_{V}$ & $2.7_{-0.7}^{+0.9}$ & $1.7_{-0.5}^{+0.6}$ & $3.0_{-0.4}^{+0.4}$ & $3.0_{-0.4}^{+0.5}$ & $2.8_{-0.1}^{+0.1}$ & $1.4_{-0.1}^{+0.1}$ \\
\hline & $s_{B}$ & $1.12(0.02)$ & $1.01(0.03)$ & $1.18(0.02)$ & $1.24(0.12)$ & $1.04(0.03)$ & $1.14(0.01)$ \\
\hline & $\chi^{2} / v$ & 1.67 & 1.50 & 1.60 & 2.83 & 0.63 & 2.38 \\
\hline \multirow{3}{*}{$\sum_{U}^{0}$} & $E(B-V)$ & $0.15(0.02)$ & $0.17(0.03)$ & $0.36(0.03)$ & $0.48(0.04)$ & $1.00(0.03)$ & $1.40(0.02)$ \\
\hline & $s_{B}$ & $1.12(0.02)$ & $1.01(0.03)$ & $1.18(0.02)$ & $1.18(0.10)$ & $1.03(0.03)$ & $1.13(0.01)$ \\
\hline & $\chi^{2} / v$ & 1.60 & 1.34 & 1.42 & 2.14 & 0.91 & 1.42 \\
\hline \multirow{3}{*}{$\begin{array}{l}\text { 总 } \\
\overline{\bar{D}} \\
\text { है } \\
2\end{array}$} & $A_{V}$ & $0.44(0.19)$ & $0.30(0.13)$ & $1.51(0.40)$ & $1.47(0.43)$ & $3.59(0.29)$ & $1.97(0.10)$ \\
\hline & $\beta$ & $-1.2(0.4)$ & $-1.7(0.5)$ & $-0.8(0.2)$ & $-1.2(0.4)$ & $-1.0(0.1)$ & $-2.0(0.1)$ \\
\hline & $t_{B}^{\max }$ & $56080.6(0.3)$ & $56191.1(0.5)$ & $56081.2(0.3)$ & $56020.4(2.7)$ & $56104.8(0.4)$ & $56688.1(0.2)$ \\
\hline
\end{tabular}

(1999, hereafter F99) law using the parameters shown in Table 5 for each SN.

A subset of the colours are also shown in Fig. 5 where the colours obtained using measurements and the $V$-band model are shown with filled and open symbols, respectively.

$\mathrm{SNe}$ Ia are intrinsically bright in the rest-frame $B$-band and this filter is commonly used to measure SN Ia distances. SN Ia light curves are typically quantified by a few parameters such as the brightness and time of maximum, $t_{B}^{\max }$, for e.g. the $B$ band, and the light-curve shape. The light-curve shape can be quantified using different methods, where perhaps the most straightforward is to measure the brightness decline for the first $15 \mathrm{~d}$ past $t_{B}^{\max }$. Phillips (1993) showed that fast declining SNe Ia are typically intrinsically fainter than slow declining objects, a discovery that lay the foundation for using SNe Ia as distance indicators for precision cosmology. An alternative approach to quantify the light-curve shape is to introduce a stretch parameter (Perlmutter et al. 1997), $s_{B}$, which can be defined as the value needed to match the time-evolution of an observed $B$ light curve to a standard SN Ia template.

The time-evolution of SN Ia $X-V$ colours also depends on lightcurve shape. For example, Nobili \& Goobar (2008, from hereon N08) present colour-light-curve shape relations in the optical while B14 show that the SNe Ia colours can be standardized over a wide range of decline rates by introducing colour-stretch, $s_{B V}=t_{B V} / 30 \mathrm{~d}$, where $t_{B V}$ is the time between $t_{B}^{\max }$ and the maximum of the $B-V$ colour. However, this approach requires $B-V$ coverage up to $t \sim t_{B}^{\max }+40$ in order to accurately determine $s_{B V}$.

We here standardize the measured colours using $s_{B}$. All colours will be studied and compared as a function of phase, $p$, defined as $p=\left(t-t_{B}^{\max }\right) / s_{B}$, where $t$ are the observing dates. The phases for the colours shown in Fig. 5 have been determined by using the values of $t_{B}^{\max }$ and $s_{B}$ from Table 5, which are fitted simultaneously with the reddening law as described in Section 4.

\section{FITTING REDDENING LAWS}

We will use a similar method to A14 to fit different parametrized reddening laws to the measured colour excesses,

$E(X-V)=(X-V)-(X-V)_{0}$, where $(X-V)_{0}$ is the assumed $X-V$ colour for the unreddened objects.

In this work, we will study different extinction laws and how well they describe the observed SN Ia reddening. Each extinction law, $A(\lambda ; \bar{a})$, depends on a set of parameters $\bar{a}$ (e.g. $E(B-V)$ and $\left.R_{V}\right)$, that will be fitted to the observed colour excesses, $E(X-V)_{p}$, by minimizing

$\chi^{2}=\sum_{X} \sum_{p} \frac{\left[E(X-V)_{p}-\left(A_{X_{p}}-A_{V_{p}}\right)\right]^{2}}{\sigma_{X_{p} V_{p}}^{2}}$.

Here $A_{X_{p}}$ and $A_{V_{p}}$ are the predicted extinctions in the $X$ and $V$ filters for phase $p$ and can be calculated as

$A_{X_{p}}=-2.5 \log _{10}\left(\frac{\int T_{X}(\lambda) \times 10^{-0.4 \times A(\lambda ; \bar{a})} \times S_{0}(\lambda ; p) \lambda \mathrm{d} \lambda}{\int T_{X}(\lambda) S_{0}(\lambda ; p) \lambda \mathrm{d} \lambda}\right)$,

if the effective filter transmission, $T_{X}(\lambda)$, and the SED, $S_{0}(\lambda ; p)$, of the unreddened source are assumed to be known. Further, $\sigma_{X_{p} V_{p}}^{2}$ are the uncertainties added in quadrature. This includes the measurement errors, but will in most cases be dominated by the intrinsic colour uncertainties discussed in Section 4.1.

In addition to the extinction law parameters $\bar{a}$, we will also minimize equation (3) with respect to the parameters $t_{B}^{\max }$ and $s_{B}$ that together determine the phase, $p$. The observed values of these parameters are extinction dependent (see e.g. Leibundgut 1988; Phillips 1993; Nugent et al. 2002; A14) since broad-band measurement of an object suffering from extinction will effectively probe redder wavelengths than the observation of the same unreddened object would have. The SN Ia light curve decline rate varies with wavelength, and slower decline rates are in general expected to be observed for reddened objects.

Similarly, the Galactic extinction, $A_{X}^{\mathrm{MW}}$, as well as the $K_{X}$ corrections, are also properties measured through broad-band filters and therefore also depend on the SED of the source, as seen from e.g. equation (4). Since the observed SED in the Milky Way is affected by the extinction in the SN host galaxy, these properties will also depend on the reddening law. The effect will be particularly significant in the UV, where the wavelength dependence for the extinction laws is steep. For example, the Galactic extinction in the Swift/uvm 2 


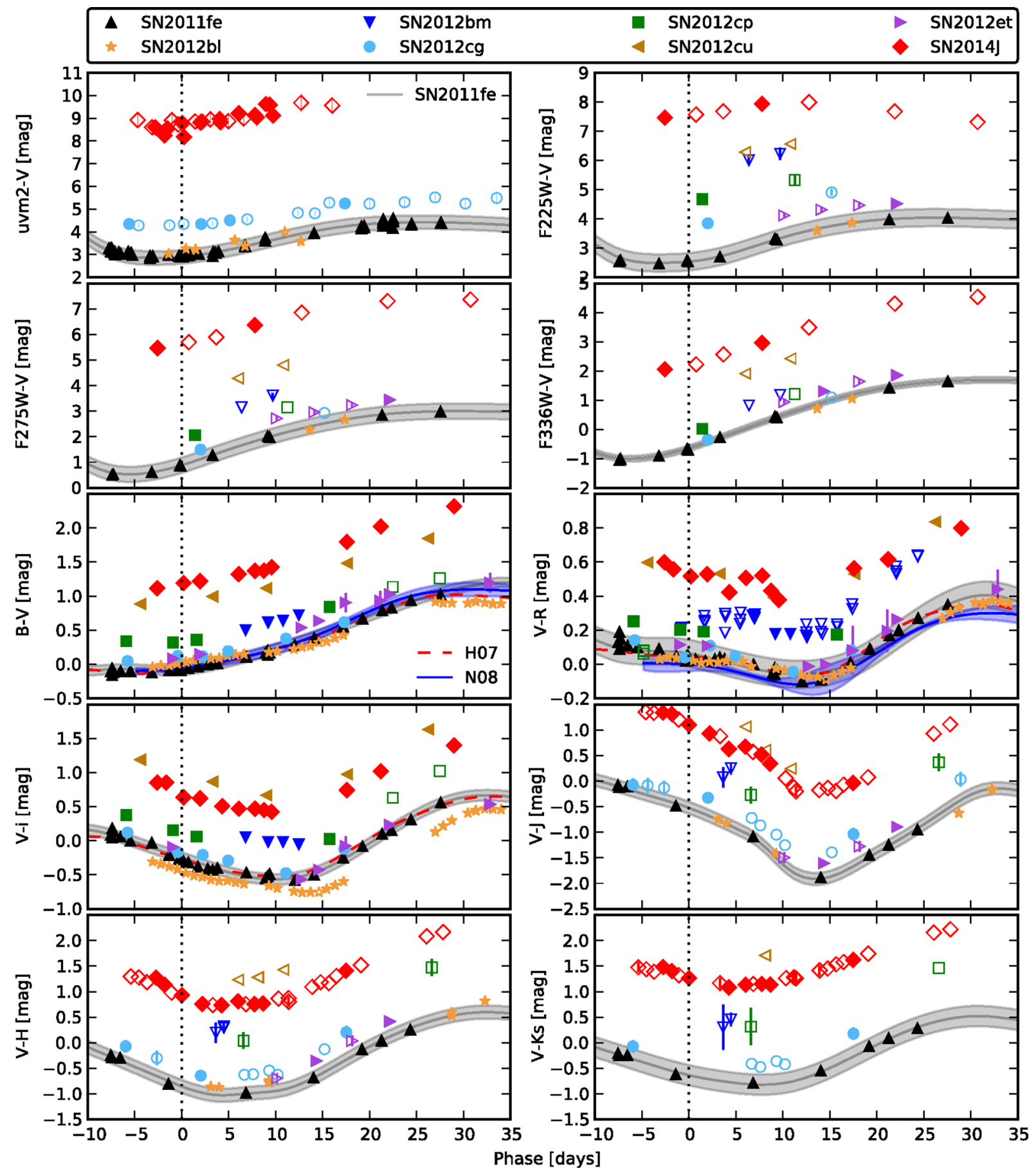

Figure 5. A selection of measured colours from UV to NIR together with literature SNe 2011by and 2011fe. The phases, $p=\left(t-t_{B}^{\max }\right) / s_{B}$, were obtained using the values of $t_{B}^{\max }$ and $s_{B}$ from Table 5. The colours from the H07 spectral template (dashed red) and Nobili \& Goobar (2008, N08, solid blue with dispersion region) are also shown together with the derived colour model of SN $2011 \mathrm{fe}$ (solid grey) with the adopted dispersions (grey region). Filled symbols show colours where a $V$-band measurement was obtained for the same date, while a $V$-band model was used to obtain the measurements that are shown with open symbols. Error bars are only plotted for data points where the uncertainty exceeds 0.1 mag. See text for details.

band for the line of sight to SN 2014J would have been $A_{X}^{\mathrm{MW}} \approx 0.3$ for an unreddened source while it is $A_{X}^{\mathrm{MW}} \approx 0.1$ for SN 2014J.

We take this effect into account by fitting each reddening law, $A(\lambda ; \bar{a})$, iteratively and update $A_{X}^{\mathrm{MW}}$ and $K_{X}$ using the fit $\bar{a}$ values in each iteration. That is, after each iteration, we re-calculate the observed colour and colour excesses using equations (1) and (2) before re-fitting the reddening law using equation (3). The procedure is repeated until the change in the fitted parameters is less than 1 per cent between iterations.

As mentioned above we use the average value of $R_{V}=3.1$ when correcting for the Galactic extinction. For the majority of the $\mathrm{SNe}$ the Galactic extinction is negligible compared to the host reddening, with the exception of SN 2012et. For this SN we also tried carrying out the fits using the extreme values of $R_{V}=2.2$ and $R_{V}=5.8$ 
observed in the Milky Way (e.g. CCM; F99) and concluded that this could impact the fit values with up to one statistical standard deviation.

\subsection{SN Ia intrinsic colours and SED}

In order to minimize equation (3), we need to make assumptions of the intrinsic colours, $(X-V)_{0}$, and $\operatorname{SED}, S_{0}(\lambda ; p)$ of the unreddened objects. The reddening laws for SNe Ia are typically derived by either comparing them to individual objects that show similarities in light-curve properties and spectral evolution (Krisciunas et al. 2006; A14), or to SN Ia colour and SED templates.

In Fig. 5, the H07 template is plotted (dashed red) for the optical colours. Although the H07 template does extend from the UV to NIR, the data it is based on are sparse at the endpoints of this range. N08 studied the intrinsic optical colours between -10 and $+50 \mathrm{~d}$ from $B$-band maximum. Two of their colour laws are shown (in blue) for normal, $s_{B}=1$, SNe Ia in Fig. 5 together with the colour dispersions they derive. Although these templates provide excellent coverage at optical wavelengths none of them cover, to high accuracy, the full wavelength range required for the UV-NIR analysis in this work.

The best studied unreddened SN Ia to date is SN 2011fe, discovered by the Palomar Transient Factory in the nearby spiral galaxy M101 (PTF11kly; Nugent et al. 2011). Its close proximity allowed detailed spectroscopic and photometric observations over a broad wavelength range from the UV (Brown et al. 2012; Mazzali et al. 2014), through the optical (e.g. Munari et al. 2013) to the near- (Matheson et al. 2012; Hsiao et al. 2013), and mid-infrared (McClelland et al. 2013). The SN was also targeted in the far-IR (Johansson et al. 2013) and radio (Chomiuk et al. 2012a), but was not detected at these wavelengths.

SN 2011fe is a normal SN Ia in both the optical and the NIR (Matheson et al. 2012; Munari et al. 2013) and does not show any spectroscopic peculiarities (Pereira et al. 2013). Further, the low Galactic and host galaxy reddening along the line-of-sight, $E(B-$ $V)_{\mathrm{MW}}=0.011 \pm 0.002 \mathrm{mag}$ and $E(B-V)_{\text {host }}=0.014 \pm 0.002 \mathrm{mag}$ (deduced from the integrated equivalent widths of the Na ID lines; Patat et al. 2013), makes it an excellent comparison object for studying reddening of SNe Ia. The colours of SN 2011fe shown in Fig. 5 (solid black lines) have been obtained by combining the available UV-NIR data, as described in Appendix B based on the measurements (black triangles). We have also combined the spectroscopic data to create a daily sampled SED, shown in Fig. B1. From the excellent $B-V$ match between SN 2011fe and H07, we can also conclude that any potential discrepancies introduced by using the SNOOPY model for the $V$-band is not likely to have any significant effect on the derived reddening laws when the colours are compared to the corresponding SN 2011 fe colours.

In order to properly compare the colours of reddened $\mathrm{SNe}$ Ia to SN 2011fe, and fit reddening laws, we also need accurate estimates of the expected intrinsic colour dispersions. It is desirable to take into account how these vary in time, and how they are correlated both in time and with different colours. However, since we lack this information for all colours involved in the analysis, we adopt a simplified approach where all colours are treated equally with phase-independent dispersions for each colour. We further follow the procedure from A14 and assume that the colour uncertainties between SN phases are completely correlated and that there is no correlation between different colours.

Milne et al. (2013, M13 from hereon) following the work of Brown et al. (2010) and Milne et al. (2010), studied the UV-optical colours of $23 \mathrm{SNe}$ Ia observed by Swift. They reported a dispersion of $\sim 0.3 \mathrm{mag}$ in $\mathrm{UV}-v$ for their $E(B-V)<0.2$ mag sample after correcting the data for extinction and the result does not seem to be affected significantly by the choice of $R_{V}$. We adopt this dispersion, shown as grey bands around the SN $2011 \mathrm{fe}$ colour template in Fig. 5, for the $u v m 2-V, F 225 W-V$, and $F 275 W-V$ colours. For the $F 336 W-V$ and $U-V$, we adopt a dispersion of $0.1 \mathrm{mag}$ based on the $U-V$ dispersion from N08. Note that since these dispersions were derived for unreddened objects, and the dispersion of SNe Ia colours decrease with wavelength, they can be considered as conservative for highly reddened $\mathrm{SNe}$. When reddened $\mathrm{SNe} \mathrm{Ia}$ are studied using the WFC3/UVIS filters, the observations will effectively probe redder wavelengths compared to similar studies of unreddened objects as illustrated in e.g. Fig. D1, and as a consequence the intrinsic dispersion of the former observations can be expected to be lower than the latter. On the other hand, even larger dispersions have been also observed in the UV, and we will use these intrinsic uncertainties under the assumption that all the $\mathrm{SNe}$ we study are similar to the reference SN $2011 \mathrm{fe}$, which we will discuss further in Section 6.1.

B14 derive a dispersion for the pseudo-colour $B_{\max }-V_{\max }$ of 0.06 mag using a Cauchy Prior for the colour distribution of their $\mathrm{SNe}$ Ia observed with the Carnegie Supernova Project. This is consistent with the phase-dependent results of N08 who derive a $B-$ $V$ dispersion of $\lesssim 0.1 \mathrm{mag}$, which we use here, within the range -10 to $+35 \mathrm{~d}$. For the $V-R$ and $V-i$ colours we adopt the N08 dispersions of $0.08 \mathrm{mag}$.

The NIR colours of SNe Ia typically show low dispersion (see e.g. Meikle 2000) and for the NIR-optical $V-J$ and $V-H$ colours we can estimate the dispersion in our phase range by using all $\mathrm{SNe}$ with $E(B-V)<0.2 \mathrm{mag}$ from the 'Gold Sample' in Stanishev et al. (2015) and find $0.12 \mathrm{mag}$ and $0.13 \mathrm{mag}$, respectively. For $V-K s$, we conservatively adopt 0.2 mag based on the studies carried out at maximum (B14) and the dispersion of the $K s$-band (Friedman 2012; Friedman et al. 2014).

The observed colour excesses, $E(X-V)$, for each reddened SN, together with the adopted colour dispersions, shown as background regions, are plotted in Fig. 6. SN 2012bl has not been plotted in the figure. It will be omitted from the extinction analysis due to its lack of reddening.

If the colour excess is observed at single wavelengths, i.e. $E\left(\lambda_{1}-\right.$ $\left.\lambda_{2}\right)=A_{\lambda_{1}}-A_{\lambda_{2}}$, it will be time-independent for interstellar dust extinction. However, for broad-band photometry, we can expect $E(X-V)$ to vary with time which is illustrated by e.g. the UV colours in Fig. 6. As the intrinsic colour of the source varies with time, the effective wavelengths of all broad-band filters will change as well. Further, since the first term on the right-hand side in equation (2) is also affected by a reddening law, any change in the effective wavelength of the filters $X$ and $V$ will affect this term more than the second term, which will induce a time-variability for $E(X-V)$. For most filters, the time-variability will be negligible except in the UV (where the extinction has a steep wavelength dependence), and in particular for filters with red tails, e.g. F225W (see Appendix D), the effect is significant. This is illustrated by the bottom panel of Fig. D1 where the effective wavelength of three WFC3/UVIS UV filters have been plotted for an SN Ia at maximum for different extinctions.

\subsection{Reddening laws}

We test three different extinction law parametrizations. In addition to the widely used CCM law modified by O'Donnell (1994, 


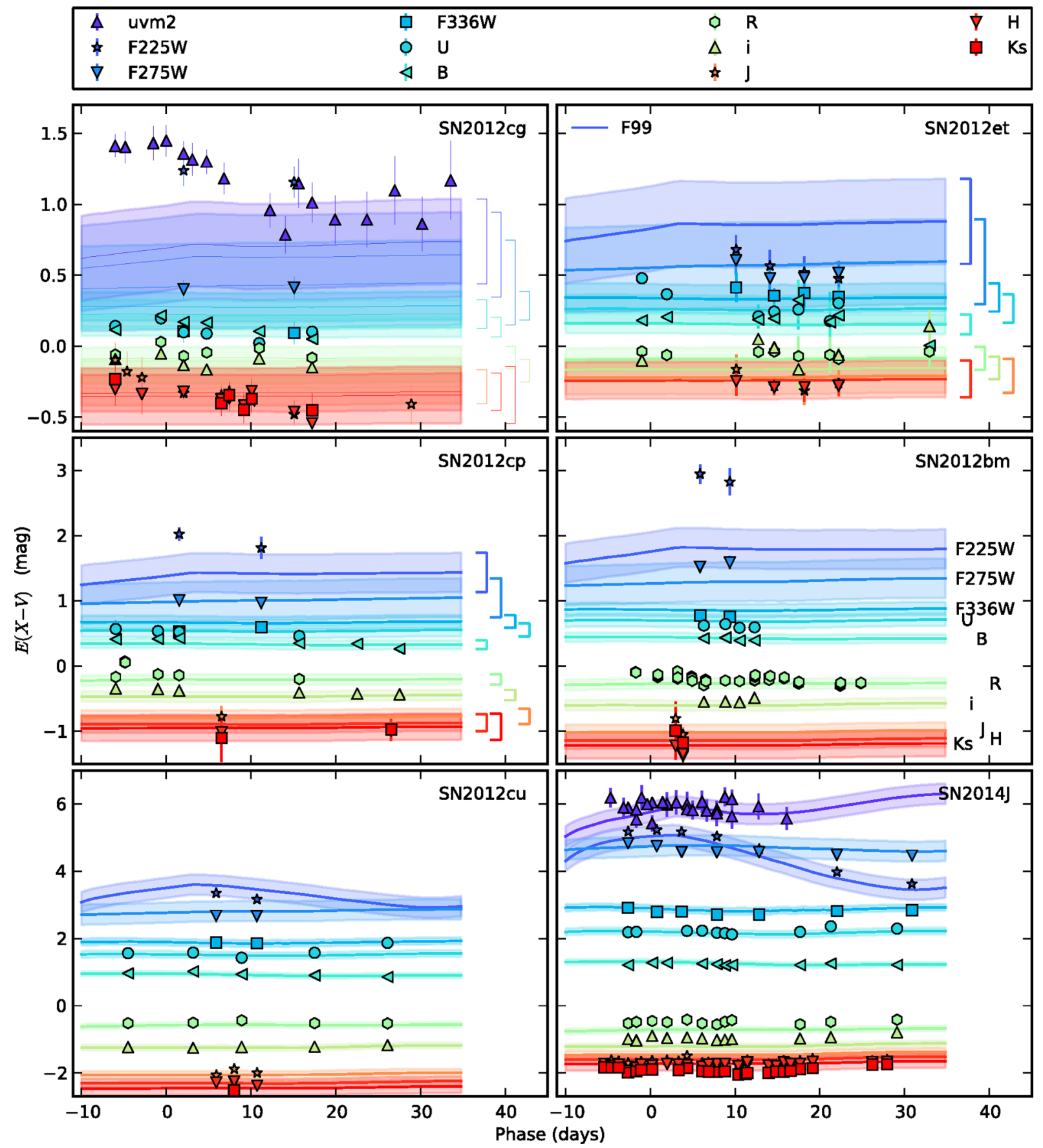

Figure 6. The observed colour excess, $E(X-V)$, against phase for all SNe and filters. The predicted colour excess from the SN 2011 fe template and the fitted F99 law, presented in Table 5, is shown with solid lines and the regions surrounding them mark the adopted intrinsic colour uncertainties. The uncertainties are also indicated by open brackets for the three SNe with the least reddening. The phase, $p=\left(t-t_{B}^{\max }\right) / s_{B}$, was calculating based on the fitted $t_{B}^{\max }$ and $s_{B}$ values from Table 5. The apparent $E(X-V)$ time-dependence of the $F 225 W$ filter for e.g. SN 2014J originates from the red tails of these filters which causes the observed colour excess to vary with the intrinsic colour of the source.

hereafter $\mathrm{CCM}+\mathrm{O}$ ), which has been derived from studying different lines of sight in the Milky Way, we also fit the parametrization from F99 that has been derived in a similar manner. For each of these we fit both the colour excess $E(B-V)$, which relates to the optical depth, and the ratio of the total-to-selective extinction, $R_{V}$. In the Milky Way the value of $R_{V}$ typically varies between $R_{V}=2.2-5.8$, for different lines of sight (e.g. CCM; F99), despite this we allow extrapolations of $R_{V}$ within the range $R_{V}=0.5-8$ when we fit the laws to the SN colours.
We also test a simple power-law model (Goobar 2008),

$A\left(\lambda ; A_{V}, a, \beta\right)=A_{V}\left[1-a+a\left(\frac{\lambda}{\lambda_{V}}\right)^{\beta}\right]$,

where the reference wavelength, $\lambda_{V}$, will be chosen as $\lambda_{V}=$ $0.55 \mu \mathrm{m}$. The CCM+O and F99 laws can be approximated by a power law in the optical and NIR range, and Goobar (2008) showed that the observed reddening law of an object embedded in CS dust 


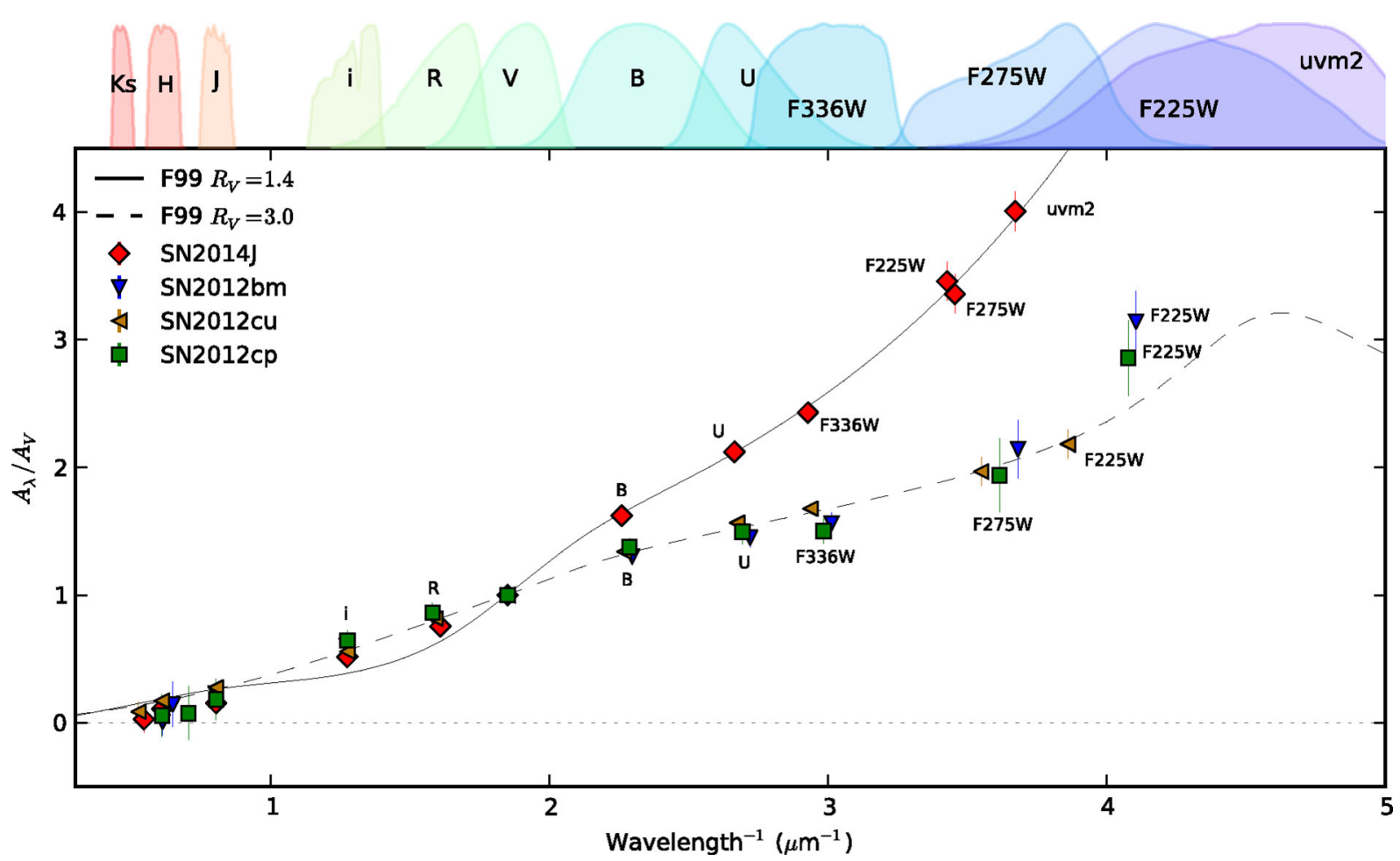

Figure 7. The normalized extinction, $A_{X} / A_{V}$ for the four SNe with $E(B-V)>0.2$ mag. Here, $A_{X} / A_{V}=1+E(X-V) / A_{V}$ where $E(X-V)$ is calculated as the weighted (with the measurement uncertainties) averages for all epochs, and $A_{V}$ is obtained from the fitted F99 parameters in Table 5. Following A14, each data point has been plotted at the wavelength where the residual to the extinction law is matching the corresponding residual between $E(X-V)$ and the fitted reddening law in Fig. 6. Note that all fitted parameters described in this work were obtained by minimizing equation (3), and not by fitting the extinction laws to the data points in this plot. The filter passbands for the filters used for the minimization are shown at the top.

can be approximated by the expression above with $a=0.8(a=0.9)$ and $\beta=-2.5(\beta=-1.5)$ for Milky Way Large Magellanic Cloud (LMC) like dust. The reason why the original extinction laws are not preserved in the CS scenario is due to the geometry where multiply scattered photons on the CS dust will reach the observer while this is extremely unlikely for an ordinary interstellar dust geometry. Note that the values in Goobar (2008) were obtained under the assumption that the light source is constant in flux and colour. For time-dependent light sources, the observed reddening is expected to vary in time which has been investigated for SNe Ia by e.g. Amanullah \& Goobar (2011).

When the power law is fitted to colour excesses, $E(X-V)=$ $A_{X}-A_{V}, A_{V}$ and $a$ are almost completely degenerate, and we are in fact only sensitive to the product $a \cdot A_{V}$. We will for this reason fix $a$ to $a \equiv 1$ in all fits below, which also has the implication that the resulting $A_{V}$ can no longer be interpreted as solemnly being the extinction in the $V$ band. We have further checked that fixing $a$ does not affect the fitted values of $\beta$, and that we obtain consistent values of the product $a \cdot A_{V}$ when $a$ is allowed as a free parameter, although the individual values of $a$ and $A_{V}$ are in this case of course poorly constrained.

In A14, the SALT2 (Guy et al. 2007b) law from Betoule et al. (2014) was also studied. This is a colour law and not an extinction law, and is further only defined for wavelengths $\lambda<8000 \AA$. It can therefore not be compared on an equal footing to the other laws and will not be studied in detail in this work. We will however compare it to the reddening or our low-extinction $\mathrm{SNe}$ in Section 6.5 .

\subsection{Results}

The fitted parameters for the three extinction laws are presented in Table 5 for each individual SN. The extinction laws were fitted to all available colours between phases -10 and $+35 \mathrm{~d}$ and the predicted colour excesses using the best fitted F99 laws are also shown in Fig. 6. The revised photometry of SN 2014J and updated intrinsic colour model with respect to A14 only had a minor impact on the fitted results for this highly reddened SN, and the values presented here are consistent with A14.

Further, all three reddening laws capture the general wavelength dependence of the observed colour excesses with the possible exception of SN 2012cg which will be discussed below. This shows that the observed SN colours from UV to NIR can indeed be described by the colours of SN 2011fe, with the adopted intrinsic dispersions, together with an extinction law with only two free parameters.

The six $\mathrm{SNe}$ span a broad range both in reddening, $E(B-$ $V)=0.2-1.4 \mathrm{mag}$, and in $R_{V}=1.4-3.0$ which confirms the findings from previous optical and NIR studies (e.g. B14), that point to a diversity of observed reddening laws of SNe Ia. The significant difference in the derived extinction laws is also illustrated in Fig. 7 where the relative extinction, $A_{\lambda} / A_{V}$, for the F99 law has been plotted for two different values of $R_{V}$. The diversity is particularly striking bluewards of the $U$ band $\left(\lambda^{-1}>2.5 \mu \mathrm{m}^{-1}\right)$, emphasizing the power of using UV data to study diversity in extinction.

In Fig. 7 we also show the relative extinction, $A_{X} / A_{V}=1+E(X$ $-V) / A_{V}$, for the four SNe with $E(B-V)>0.2$ mag against inverse wavelength. Here, $E(X-V)$ is calculated as the weighted average for all phases and $A_{V}$ was obtained from the fitted F99 parameters 


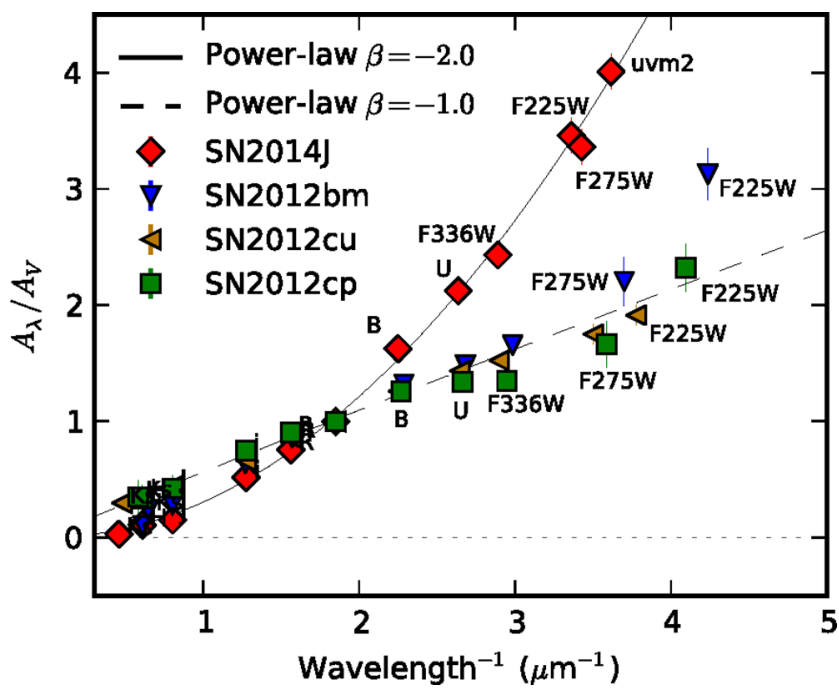

Figure 8. Similar to Fig. 7 but here the power-law fits from Table 5 are shown with the observed reddening. The wavelengths of the data points will differ from Fig. 7 due to the way these plots are created, as explained in the text. Note that all fitted parameters described in this work were obtained by minimizing equation (3), and not by fitting the extinction laws to the data points in this plot.

as $A_{V}=R_{V} \cdot E(B-V)$. Because of this, the derived values for $A_{X} / A_{V}$ are model-dependent and will be different for the different extinction laws. Further, since the extinction $A_{X}$ is obtained from comparing observations in the filter $X$ of reddened and unreddened sources, and the measurements correspond to different effective wavelengths, it is non-trivial to derive a specific wavelength corresponding to $A_{X} / A_{V}$. For these reasons, we follow the procedure from A14 and plot the $A_{X} / A_{V}$ points in a manner where we chose to preserve the residuals between the fitted model and the data points from Fig. 6. This is obtained by plotting each point, $A_{X} / A_{V}$, at the wavelength where the residual to the extinction law is the same as the corresponding weighted average residual of $E(X-V)$ over all epochs. We emphasize that this plot is only a way of visualizing the data and the reddening laws and that all extinction parameters were obtained by minimizing equation (3), i.e. not by fitting the extinction laws to the data points in Fig. 7.

One observation that can be made by comparing the results in Table 5 and Fig. 6 is that although all extinction laws appear to be general enough to describe all SNe, the F99 law shows tension for SNe 2012bm and 2014J. These SNe have in common that they both show a fair amount of reddening $(E(B-V)>0.5 \mathrm{mag})$. The more dust along the line of sight to an SN, the more sensitive the fit will be to the extinction law, and less to possible intrinsic SN colour variations. In other words, we can expect any deviation between the assumed parametrization and the actual observed extinction law to become more significant the redder the $\mathrm{SNe}$ are. It has already been pointed out that since the F99 law is empirical in nature, it may have features that do not properly extrapolate to low values of $R_{V}$. This could be the case for SN 2014J, where the F99 law with $R_{V}=1.4$ leads to a more prominent feature around $\sim 1.5 \mu \mathrm{m}^{-1}(\sim 6700 \AA)$ than what is supported by the data. In Fig. 8, we compare the powerlaw fits for the four most reddened $\mathrm{SNe}$, and here we can see how this is consistent with all the data points over the full wavelength range for SN 2014J.

From the reduced $\chi^{2}$ in Table 5 we can conclude that the powerlaw model provides better fits than F99 for both low- $R_{V}$ SNe, 2012et and 2014J, while the opposite is true for the $R_{V} \lesssim 3 \mathrm{SNe} 2012 \mathrm{cg}$,
Table 6. Fitted F99 parameters using only data between -5 to +5 and +10 to $+20 \mathrm{~d}$ from maximum, respectively. While SNe 2012cg and 2014J both have UV-NIR observations for both intervals, the fits to SN 2012cp are only based on UVoptical data. The parameters $t_{B}^{\max }$ and $s_{B}$ were fixed to the values given in Table 5 .

\begin{tabular}{lcccc}
\hline SN & Phase & $E(B-V)$ & $R_{V}$ & $\chi^{2} / v$ \\
\hline SN2012cg & {$[-5,+5]$} & $0.16(0.02)$ & $1.7_{-0.6}^{+0.8}$ & 1.51 \\
& {$[+10,+20]$} & $0.13(0.03)$ & $3.6_{-1.0}^{+1.5}$ & 1.34 \\
SN2012cp & {$[-5,+5]$} & $0.33(0.03)$ & $1.8_{-0.5}^{+0.6}$ & 1.63 \\
& {$[+10,+20]$} & $0.34(0.03)$ & $2.2_{-0.6}^{+0.8}$ & 0.55 \\
SN2014J & {$[-5,+5]$} & $1.35(0.03)$ & $1.5_{-0.1}^{+0.1}$ & 1.81 \\
& {$[+10,+20]$} & $1.32(0.03)$ & $1.6_{-0.1}^{+0.1}$ & 1.56 \\
\hline
\end{tabular}

$2012 \mathrm{cp}$ and $2012 \mathrm{cu}$. In particular, the F99 and CCM+O law seem to provide nearly perfect fits to the observations of SN 2012cu, which is also seen in Fig. 7. SN 2012bm shows tension for all fitted laws.

The $\mathrm{CCM}+\mathrm{O}$ law performs better in terms of goodness-of-fit for several cases, e.g. for SN 2014J. However, this law does not extrapolate well to low- $R_{V}$ values which give rise to a sharp, discontinuous, knee when the NIR and optical parametrizations of the law are merged.

\section{SEARCHING FOR CS DUST}

All the SNe studied here show a colour excess evolution that is within the adopted dispersion with the exception of SN 2012cg. This SN reveals a minimum in its NIR colour evolution around two weeks past max which is possibly accounted to the diversity observed in NIR SN Ia light curves between the first and second peaks. This is within the assumed intrinsic uncertainty although the assumption that these are fully correlated between epochs does not hold in this case. What is perhaps even more striking are the $\sim 2 \sigma$ variations of the $u v m 2-V$ evolution which suggests that this colour evolve slower than the corresponding $2011 \mathrm{fe}$ colour, resulting in an apparent decrease with time of the colour excesses.

The fact that the observed colour evolution of SN 2012cg is not consistent with a single extinction law is also illustrated by Table 6 where the F99 law has been fitted for two phase intervals: around maximum and between 10 and $20 \mathrm{~d}$ past $t_{B}^{\max }$. The fits were carried out for the three SNe that had UV data in both these intervals while keeping the fitted values of $t_{B}^{\max }$ and $s_{B}$ fixed to the values given in Table 5. The fitted parameters of SNe 2012cp and 2014J are within errors between the two epochs while this is not the case for SN 2012cg.

Evolving colour excesses, or a reddening law that changes with time, is predicted if CS dust is present in the SN environment. Amanullah \& Goobar (2011) studied this effect at optical wavelengths and we can extend that analysis, using the same tools, to also cover UV and NIR in order to test the observed colour evolution of SN 2012cg against the expectations for a CS scenario.

In Fig. 9 the same colour excesses of SN 2012cg that are plotted in the upper-left panel of Fig. 6 are shown. The solid lines represent the result when we fit a combined F99 and CS dust law to the bluest colours, $u v m 2-V$ and $F 225 W-V$, while keeping $t_{B}^{\max }$ and $s_{B}$ fixed to the values from Table 5. The best-fitting values for the F99 law are $E(B-V) \approx 0.2$ mag and $R_{V} \approx 3.0$. The CS dust in this case consists of dust modelled to match the average extinction properties in the Milky Way (Draine 2003) and distributed in a thin spherical 


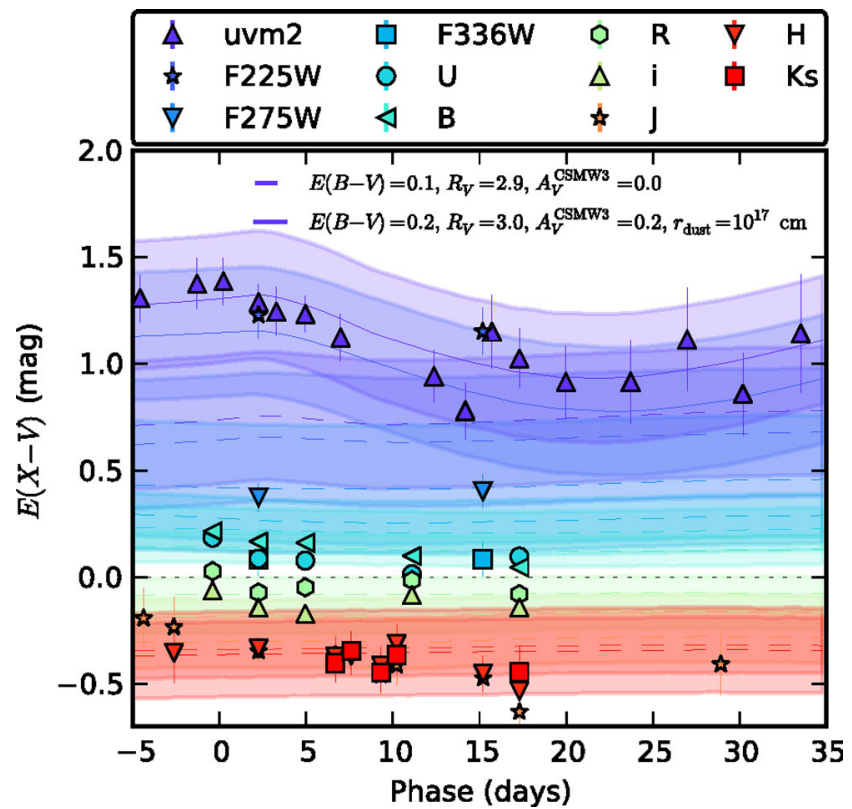

Figure 9. The measured colour excesses for different phases for SN $2012 \mathrm{cg}$ are taken from Fig. 6. Overplotted are the best fits of a combined CS and F99 dust law, where the former has been adopted from Amanullah \& Goobar (2011) where the dust is distributed in a thin spherical shell. The solid lines show the best fit when only the $u v m 2-V$ and $F 225 W-V$ colours are considered, while the dashed lines show the fit to all colours. The former is obtained with a CS dust shell at radius $r_{\text {dust }} \sim 10^{17} \mathrm{~cm}$ from the SN that give rise to an extinction of $A_{V} \sim 0.2$, while latter fit is consistent with no CS dust.

shell. Here, the best fit yields an extinction of $A_{V}^{\text {CSMW3 }} \approx 0.2$ for a shell at a radius of $r_{\text {dust }} \sim 10^{17} \mathrm{~cm}$.

Although adding Milky Way-like CS dust to the picture can give an explanation for the time-evolution of the bluest colours, it does not provide an improvement beyond a single F99 law when all colours are considered simultaneously, which is shown by the dashed lines in Fig. 9. Here, the best fit is obtained for $A_{V}^{\text {CSMW3 }} \approx$ 0.0. The explanation for this is that the specific CS dust model we test also predicts evolution of the redder colours which in this case is not supported by the data. If CS dust is present, the total extinction, $A_{\lambda}$, can only decrease with time due to late-arriving scattered photons. From the results in Table 6, we see that this is not the case for $A_{V}$, although the value of $A_{V}$ is in this case model dependent.
It is also possible to detect CS dust by studying the time-evolution of multi-epoch high-resolution spectroscopy. This was mentioned in Section 1 and is the observational signature that has been used to claim detections of CS dust around SNe Ia.

For SN 2012cg, we obtained high-resolution FIES spectra for three epochs which are listed in Table 4 and correspond to phases $-7,1$ and $13 \mathrm{~d}$ with respect to $t_{B}^{\max }$. All spectra contain wellresolved unsaturated $\mathrm{Na}$ ID features (Fig. 4), which are shown in Fig. 10. The bulk of the distinguishable features are likely part of the visible interstellar medium (ISM), but it cannot be excluded that $\mathrm{Na}$ I associated with CS medium could contribute to the observed profile. A full description of the Na ID profile and summary of other interstellar absorption features is presented in Section 6.4.

The FIES spectra were normalized by fitting third-order polynomials to the continua bracketing $\mathrm{Na}$ ID. No telluric corrections were performed on the spectra, due to the lack of standard star observations on some of the epochs. We fitted Gaussian profiles to the weighted average of the three epochs, and find that the Na ID line ratios (D1/D2) range from 2 to 1.2 for individual features. The deepest features have low ratios, indicating that they are not optically thin. Based on a standard star spectrum taken on the second epoch, we identify telluric features overlapping with $\mathrm{Na}$ ID2, whereas $\mathrm{Na}$ ID1 appears to be located at a less affected part of the spectrum. We therefore focus most of the time-variation analysis on $\mathrm{Na}$ ID1, because it is less contaminated by telluric features and it is more likely to be optically thin due to the lower absorption cross-section of this transition. We measure the total equivalent width of $\mathrm{Na}$ ID1 of the respective epochs to be, $702 \pm 9,711 \pm 32$ and $685 \pm 20 \mathrm{~mA}$.

Since no significant time-variations of $\mathrm{Na}$ ID absorption between different epochs could be detected beyond the noise level, we attempt to use the non-detections to constrain possible CS dust models assuming a thin sphere of CS material shell distribution around the $\mathrm{SN}$. Using the Na I photo-ionization model described by Borkowski et al. (2009) and recently applied to SN 2014J in Graham et al. (2015b), we can exclude CS material, and thus also CS dust, at certain radii from the SN. If the $\mathrm{Na}$ I is optically thin, a change in equivalent width of $\mathrm{Na} \mathrm{ID}$ is directly proportional to a decrease in column density. Furthermore, the product of the integrated photon count flux above the ionization energy of $\mathrm{Na}$ I (2400 $⿱$ A) and its corresponding ionization cross-section is proportional to the fractional decrease in column density. We can thus compute the fractional decrease in equivalent width of $\mathrm{Na} \mathrm{ID}$ expected due to photoionization of CS material at a given radius from an SN. In this model, $\mathrm{CS} \mathrm{Na}$ I can be excluded which is far enough away from the SN to not have been fully ionized by the first epoch and close enough to see changes by the following epochs. The diversity of SNe Ia

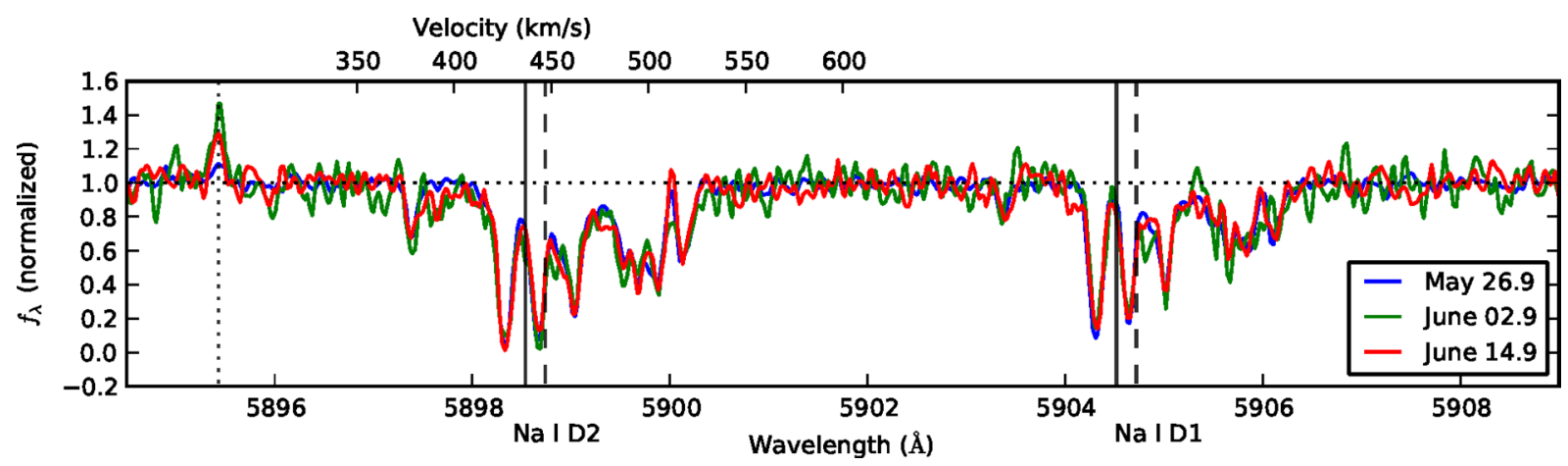

Figure 10. Na ID absorption doublet of SN 2012cg. The rest-frame wavelength of Na ID in NGC 4424 (solid lines) and for the approximate stellar velocity along the line of sight (dashed) are indicated. The dotted vertical line indicates a sky emission line. In Section 5 the profile of Na ID is discussed in detail. 


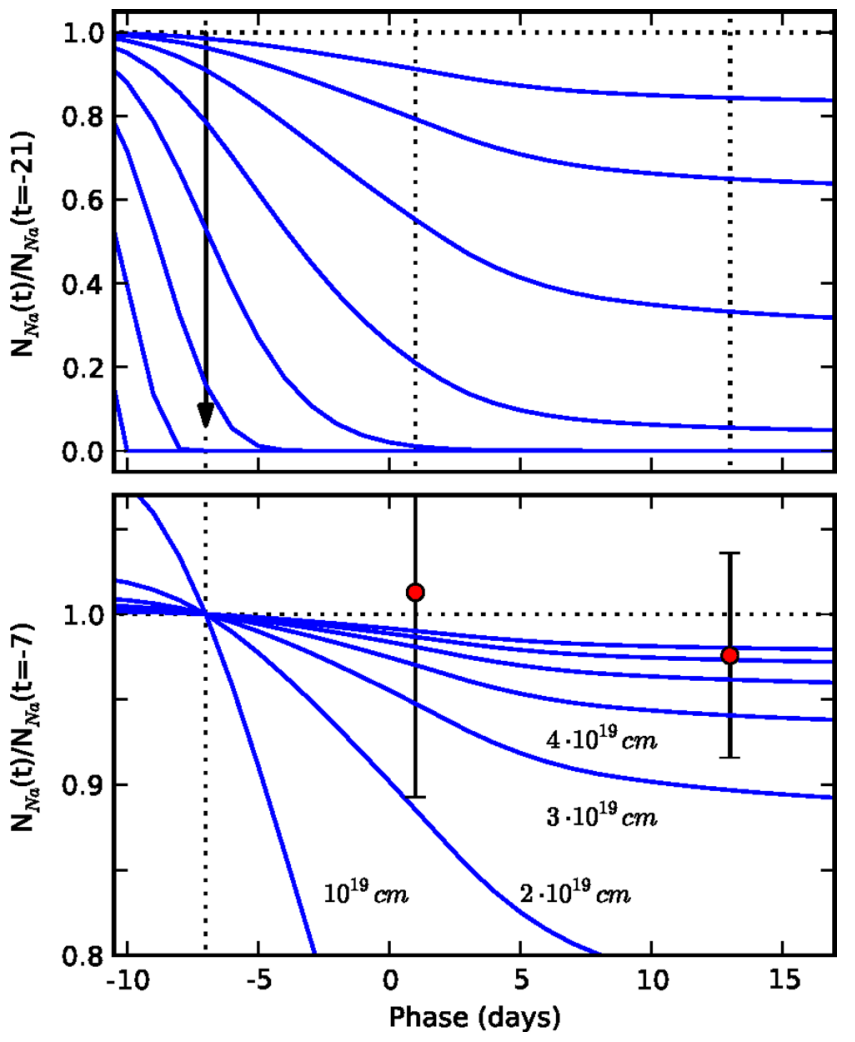

Figure 11. Na I ionization models for thin shells of CS material at different distances from an SN, suggesting the absence of CS material at distances between $r_{\text {dust }}=2 \times 10^{18}-3 \times 10^{19} \mathrm{~cm}$ around SN 2012cg. The upper panel shows the fractional decrease of $\mathrm{Na}$ I due to ionization as a function of time at radii ranging from $r_{\text {dust }}=10^{18}-3 \times 10^{19} \mathrm{~cm}$. The arrow indicates an approximate upper limit for $\mathrm{Na}$ I around $2012 \mathrm{cg}$ to be ionized beyond detection level by $-8 \mathrm{~d}$ from $B$ maximum based on the $\mathrm{S} / \mathrm{N}$ around the Na ID doublet of the first FIES spectrum. The lower panel shows the fractional change of $\mathrm{Na}$ I with respect to the first epoch. The fractional change of equivalent width of $\mathrm{Na}$ ID1 with $3 \sigma$ error bars set an approximate lower radius limit at which $\mathrm{CS}$ material could be present while the ionization effects remain undetected.

spectra in UV implies that the results will be sensitive to the spectral templates used. Noting that the STIS spectra of SN 2012cg resemble those of SN 2011fe, we use the SN 2011fe spectral template described in Appendix B. However, the H07 template extends to shorter wavelengths and earlier times than the SN 2011fe template. We therefore extrapolate our spectral template by H07, whereby the flux is rescaled for continuity. This slightly increases the photoionizing flux.

In Fig. 11, the model fractional decrease of $\mathrm{Na}$ I at different radii from an SN are shown. We determined the inner radius of excluded CS Na I of 2012cg by assuming that an absorption feature deeper than three times the RMS of the noise would be detectable in the earliest spectrum. This yields a distance of $r_{\text {dust }}<2 \times 10^{18} \mathrm{~cm}$ within which the Na I column density would have decreased below detection levels since explosion. With respect to the first epoch, the fractional change of the equivalent width of $\mathrm{Na}$ ID1 is considered to set an upper limit on the change in column density. Within $3 \sigma$ errors, the last epoch excludes the models in which the dust is located closer than $r_{\text {dust }}>3 \times 10^{19} \mathrm{~cm}$ from SN 2012cg. Using the total equivalent width of $\mathrm{Na}$ ID1 of course has the caveat that it considers the entire measured column density to be situated at one radius from the $\mathrm{SN}$, which we know cannot be true based on the profile. We therefore also consider how much a single feature must change to be detected given the signal to noise of the spectra. The limits obtained by the absence of change beyond three times the RMS noise are comparable to those set by the fractional change of the total equivalent width. Lastly, we note that the limits are not valid if there is CS material that is not optically thin, since the equivalent width then does not change linearly with column density.

In summary, although the existence of Milky Way-like CS dust at $r_{\text {dust }} \sim 10^{17} \mathrm{~cm}$ is consistent with the observed time-evolution of $E(u v m 2-V)$ and non-detection of time-varying Na ID absorption for SN 2012cg, the minimal $E(X-V)$ evolution of the redder wavelengths disfavours this explanation. This suggests that CS dust can only be present either in very small amounts, $A_{V}^{\text {CSMW3 }}<0.05$ or if it is located at larger radii $r_{\text {dust }} \sim 10^{19} \mathrm{~cm}$ for which the timedelays are much larger than the time-scales studied here and are not expected to affect the observed colours (Amanullah \& Goobar 2011). Further, non-detections in radio (Chomiuk et al. 2012b) and far-IR (Johansson et al. 2013) are other observational signatures suggesting that SN 2012cg exploded in an environment free from CS material. Here, the far-IR observations are sensitive to preexisting CS dust that would be heated by the explosion.

\section{DISCUSSION}

\subsection{Using SN 2011fe as a colour template}

One possible explanation for the time-evolution of the SN 2012cg $U V-V$ colour excess could be that it is intrinsically different from SN $2011 \mathrm{fe}$. We have already argued that all the SNe in our sample, are normal SNe Ia by comparing their overall spectra (see Fig. 4) and their SNID classification, but we can also study individual features which has proven to be a useful approach for sub-classifying normal $\mathrm{SNe}$ Ia.

The properties of individual features can be quantified by e.g. measuring their velocities and pseudo-equivalent widths $(p W)$, where pseudo refers to the fact that the equivalent widths are obtained using a pseudo-continuum since proper continua are absent in $\mathrm{SN}$ Ia spectra. The pseudo-continuum can be defined with a straight line between the two flux peaks surrounding an absorption feature and the $p W$ is then calculated as the integral of the spectrum flux relative to the continuum. Using this method, the error of the measurement will typically be dominated by the systematic uncertainty introduced by the determination of the pseudo-continuum.

Branch et al. (2006) suggested a classification scheme based on pseudo-equivalent width measurements of the absorption features near $5750 \AA$ and $6100 \AA$ (following the naming convention from Garavini et al. 2007, we refer to these as $p W 6$ and $p W 7$, respectively), that can be associated with $\mathrm{Si}$ II $\lambda 5972 \AA$ and $\mathrm{Si}$ II $\lambda 6355$ $\AA$ as shown in Fig. 4. They identify four different groups when considering $p W 6$ and $p W 7$ for their sample where in particular the 'core normal' (CN) SNe, to which SN 2011fe belongs, are tightly clustered and show a high degree of general spectral homogeneity.

In Table 7, we show measurements of $p W 6$ and $p W 7$ for spectra close to maximum after correcting our spectra for host galaxy reddening (although this is not expected to have any major impact on the results as discussed in Nordin et al. 2011) using the fitted reddening laws from Section 4.3. We also present the $p W$ for $\mathrm{Ca}$ II $\mathrm{H} \& \mathrm{~K}$ ( $p W 1$ following Garavini et al. 2007) and note that all $\mathrm{SNe}$ have $p W 1<150 \AA$. It has been shown that $p W 1$ correlates with the intrinsic $U-B$ colour (Folatelli et al. 2013; B14), where SNe with $p W 1>150 \AA$ are intrinsically redder than the bulk of normal SN Ia. 
Table 7. Calculated pseudo-equivalent widths $(p W)$ based on the optical spectra. The $p W$ naming convention from Garavini et al. (2007) is followed with $p W 1$ corresponding to $\mathrm{Ca}$ II $\mathrm{H} \& \mathrm{~K}$, and $p W 6$ and $p W 7$ corresponding to $\mathrm{Si}$ II $\lambda 5972 \AA$ and $\mathrm{Si}$ II $\lambda 6355 \AA$, respectively. The quoted uncertainties are the statistical errors. Systematic uncertainties from estimating the pseudo-continuum could be dominating in some cases. The classification as 'core normal' $(\mathrm{CN})$ or 'broad-line' (BL) is based on the criteria from Blondin et al. (2012). Given that these criteria were derived based on spectra around maximum, the classification of SN 2012cu should be considered less certain. The measurement of SN 2014J was carried out on a spectrum published in Goobar et al. (2014). See the text for details.

\begin{tabular}{lccccc}
\hline SN & $\begin{array}{c}\text { Phase } \\
(\text { days })\end{array}$ & $\begin{array}{c}p W 1 \\
(\AA)\end{array}$ & $\begin{array}{c}p W 6 \\
(\AA)\end{array}$ & $\begin{array}{c}p W 7 \\
(\AA)\end{array}$ & Type \\
\hline $2011 \mathrm{fe}$ & +0 & $94(1)$ & $14(1)$ & $94(1)$ & $\mathrm{CN}$ \\
$2012 \mathrm{bl}$ & -1 & $110(2)$ & $1(1)$ & $89(2)$ & $\mathrm{CN}$ \\
$2012 \mathrm{bm}$ & +9 & $125(2)$ & $13(1)$ & $76(2)$ & $\mathrm{CN}$ \\
$2012 \mathrm{cg}$ & +2 & $103(4)$ & $9(3)$ & $75(4)$ & $\mathrm{CN}$ \\
$2012 \mathrm{cp}$ & +2 & $98(1)$ & $10(1)$ & $74(1)$ & $\mathrm{CN}$ \\
$2012 \mathrm{cu}$ & +9 & $70(1)$ & $24(1)$ & $99(1)$ & $\mathrm{CN}$ \\
$2012 \mathrm{et}$ & +2 & $123(2)$ & $11(1)$ & $133(2)$ & $\mathrm{BL}$ \\
$2014 \mathrm{~J}$ & -1 & $155(2)$ & $14(1)$ & $100(3)$ & $\mathrm{CN}$ \\
\hline
\end{tabular}

Using the classification criteria from Blondin et al. (2012), we can classify all SNe as CN except for SN 2012et which lands in range that Branch et al. (2006) defines as the 'broad-line' (BL) group. These SNe Ia have broader and deeper $6100 \AA$ absorption but are in most aspects not very different from $\mathrm{CN} \mathrm{SNe}$. When the observed spectra from the two groups are compared with synthetic spectra generated with SYNOW (Branch et al. 2003) similar photospheric velocities and excitation temperatures can be used to describe both the $\mathrm{CN}$ and BL groups (Branch et al. 2006).

Given these results, there is no evidence for any significant discrepancy between the optical properties of our SNe and SN 2011fe, with the exception for the additional source of luminosity observed in the very early optical light curve of SN 2014J (Goobar et al. 2015). However, several studies have shown that normal SNe in the optical still show dispersion in the UV and M13 argued that their sample could be divided in up to four groups. Here, SN $2011 \mathrm{fe}$ belongs to what they define as the 'near-UV (NUV) blue' group which they conclude is on average 0.44 mag bluer in $u-v$ than the reddest, 'NUV-red', group. They consider SNe with $E(B-$ $V)<0.25 \mathrm{mag}$, and this constraint could still allow extinction to contribute with up to $0.9-1.3 \mathrm{mag}$ (depending on the extinction law) to the $u v m 2-v$ colour, which may offer an explanation for the significant colour dispersion. However, M13 show that the two groups are distinguishable even after the individual SNe have been corrected for reddening.

Further, they also conclude that the groups, on average, show different spectroscopic properties. All NUV-blue SNe have 'normal' photospheric expansion velocities around maximum when quantified based on the Si II $\lambda 6355 \AA$ feature, while the remaining groups are populated with both NV and HV SNe. They use the definition from Wang et al. (2009), where SNe with Si II velocities of $v_{\text {Si II }}>11800 \mathrm{~km} \mathrm{~s}^{-1}$ at maximum are classified as $\mathrm{HV}$ and the remaining as NV. Although there may not be a sharp distinction between the two classes (see e.g. Silverman, Kong \& Filippenko 2012a), the M13 results are consistent with previous studies showing that an optical colour-velocity dependence exists with HV SNe being on average intrinsically redder (Foley \& Kasen 2011; Blondin et al. 2012; Folatelli et al. 2013; Mandel, Foley \& Kirshner 2014).

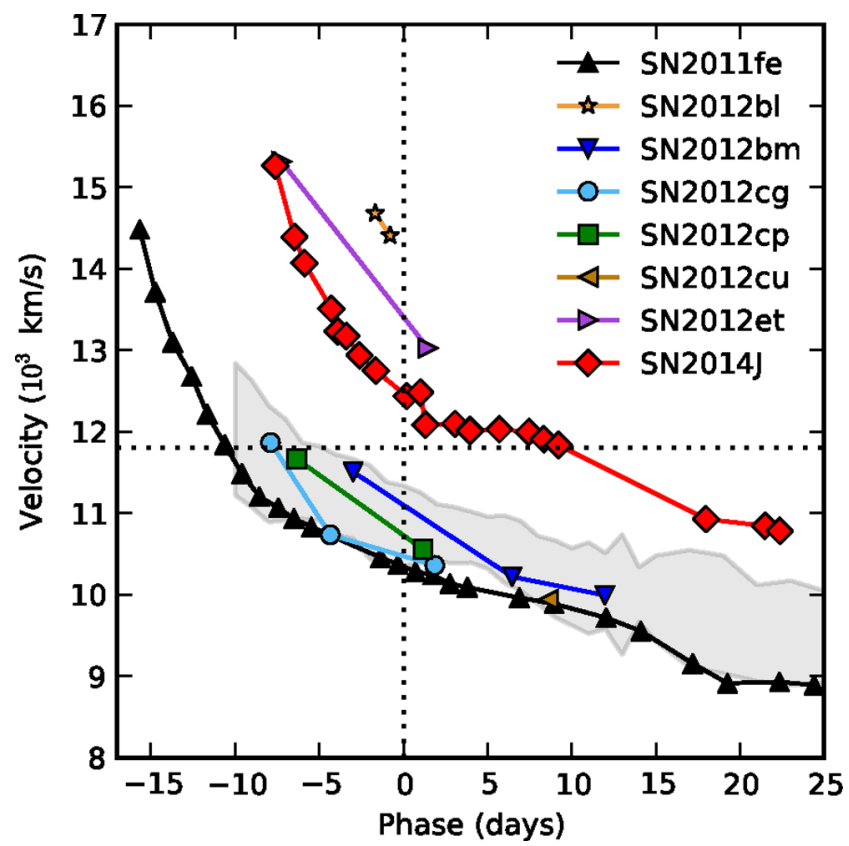

Figure 12. Measured velocities of Si II $\lambda 6355$ for different phases of the SNe studied in this work together with reference objects. For SNe $2011 \mathrm{fe}$ and 2014J the velocities were calculated based on the spectra published by Pereira et al. (2013), Goobar et al. (2014) and Marion et al. (2015). The grey region is marking the average SN Ia velocity range (Folatelli et al. 2012, 2013). The dashed line marks the difference used to separate highand low-velocity objects by Wang et al. (2009) at maximum.

On the other hand, exceptions from these findings is, SN 2011de, the brightest SN Ia observed (Brown 2014), and the results by Wang et al. (2012), showing, using the HST Advanced Camera for Surveys, that the UV-optical colours of the HV object SN 2004dt are significantly bluer than the NV SN 2005cf.

We have measured the Si II velocities, which are presented in Fig. 12, for all spectra we have obtained of the SNe at $t<+25 \mathrm{~d}$ from $t_{B}^{\max }$ by fitting Gaussian profiles. From the figure we conclude that SNe 2011fe, 2012bm, 2012cg and 2012cp show similar velocity evolutions, and the single measurement we have of SN 2012cu is also consistent with SN 2011fe. The SNe 2012bl, 2012et and $2014 \mathrm{~J}$, on the other hand, are classified as HV. Note however that the velocity range for the full sample is still small enough for the scatter induced by the optical colour-velocity relation to land within the adopted uncertainty of 0.1 mag for $B-V$ (Mandel et al. 2014). Mandel et al. (2014) further find no evidence for such a relation for the $V-R$ and $V-i$.

SN 2012et is both classified as BL (although the $p W 6$ and $p W 7$ values put its close to the border of the $\mathrm{BL}$ and $\mathrm{CN}$ groups) and $\mathrm{HV}$, and such objects have been shown to be redder than the bulk of normal SNe Ia (Folatelli et al. 2013; B14). However, the value of $p W 1$ for SN 2012et at $-8 \mathrm{~d}$ is $p W 1=82 \pm 7 \AA$, which is well below $p W 1 \sim 150 \AA$ 品here the BL HV start to deviate in colour (see fig. 17 in B14).

M13 also determine that NUV-blue SNe show evidence of unburned $\mathrm{C}$ II in their optical spectra while the remaining groups consist of objects both with and without unburned $\mathrm{C}$ II detections. These results are also consistent with optical spectroscopic studies where SNe Ia with signatures of unburned carbon are bluer in their optical colours (e.g. Thomas et al. 2011; Folatelli et al. 2012; Silverman \& Filippenko 2012). Unburned carbon can be detected through absorption attributed to the most prominent line in the optical, $\mathrm{C}_{\text {II }}$ 


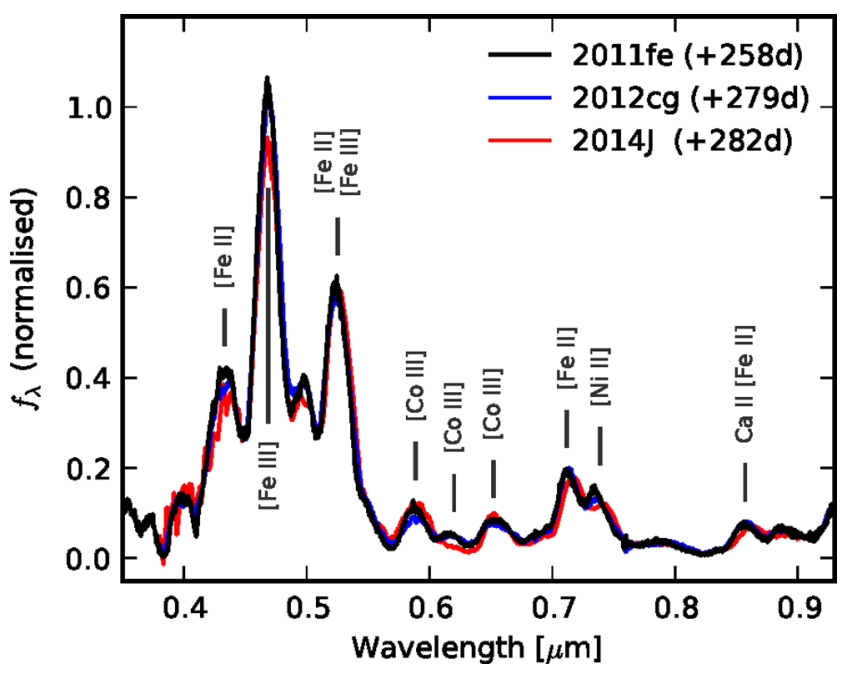

Figure 13. Nebular spectra of SNe 2011fe, 2012cg and 2014J obtained with the William Herschel Telescope, the Keck and the Apache Point Observatory. The epochs of the spectra with respect to maximum is specified in the legend. The spectra of SNe 2012cg and 2014J were corrected for reddening and smoothed using a 5 pixel boxcar, and then scaled to match the spectrum of SN 2011fe. The main features of the nebular spectra have been marked.

$\lambda 6580$, but the prospects of detecting this line decreases for epochs approaching maximum light (see e.g. fig. 11 in Folatelli et al. 2012). The SNe 2011fe, 2012cg and 2014J have all evidence for unburned material in their spectra (Nugent et al. 2011; Silverman et al. 2012b; Marion et al. 2015), while the first spectra of the remaining objects were all obtained $>-7 \mathrm{~d}$ with respect to $t_{B}^{\max }$.

Finally, we can also compare the optical spectra of SNe 2011fe, $2012 \mathrm{cg}$ and $2014 \mathrm{~J}$ at the late-time nebular phase when the material in the SN ejecta is optically thin. In Fig. 13, we present spectra of the three $\mathrm{SNe}$ at 260-280 d past maximum after they have been corrected for reddening. The spectra are dominated by forbidden lines from the decay chain ${ }^{56} \mathrm{Ni} \rightarrow{ }^{56} \mathrm{Co} \rightarrow{ }^{56} \mathrm{Fe}$. The spectra of $\mathrm{SNe}$ $2012 \mathrm{cg}$ and $2011 \mathrm{fe}$ are remarkably similar while for SN 2014J the [Co III] line at $6200 \AA$ is suppressed and the features at 7000-7400 $\AA$ are redshifted with respect to the others. The two peaks identified as [Fe II] $\lambda 7155$ and [Ni II] $\lambda 7378$ (e.g. Maeda et al. 2010a,b), are consistently blueshifted with respect to their rest frame by 800 $1000 \mathrm{~km} \mathrm{~s}^{-1}$ in SNe $2011 \mathrm{fe}$ and $2012 \mathrm{cg}$. In contrast, these lines appear redshifted in SN 2014J by a similar amount. This is in line with the findings of Maeda et al. (2010a) that SNe showing an HV gradient (Benetti et al. 2005), generally associated with HV SNe (Wang et al. 2009), demonstrate exclusively redshifted nebular velocities, while $\mathrm{LV} \mathrm{SNe}$ preferentially demonstrate blue shifted nebular profiles.

In conclusion, based on the optical spectroscopy available both around maximum light and in the nebular phase, there are no observational signatures that would disfavour comparing the colours of the SNe to SN 2011fe for studying reddening together with the adopted uncertainties. In particular, while SNe $2011 \mathrm{fe}$ and $2012 \mathrm{cg}$ have slightly different light-curve shapes, they show remarkable similarities in spectroscopical features and evolution. However, the SNe 2011 by and $2011 \mathrm{fe}$ have also been found to be almost identical in the optical while showing significant discrepancies in the UV (Foley \& Kirshner 2013; Graham et al. 2015a). Next we investigate the impact on the fitted reddening laws if we instead compare the measured colours to SN 2011by.

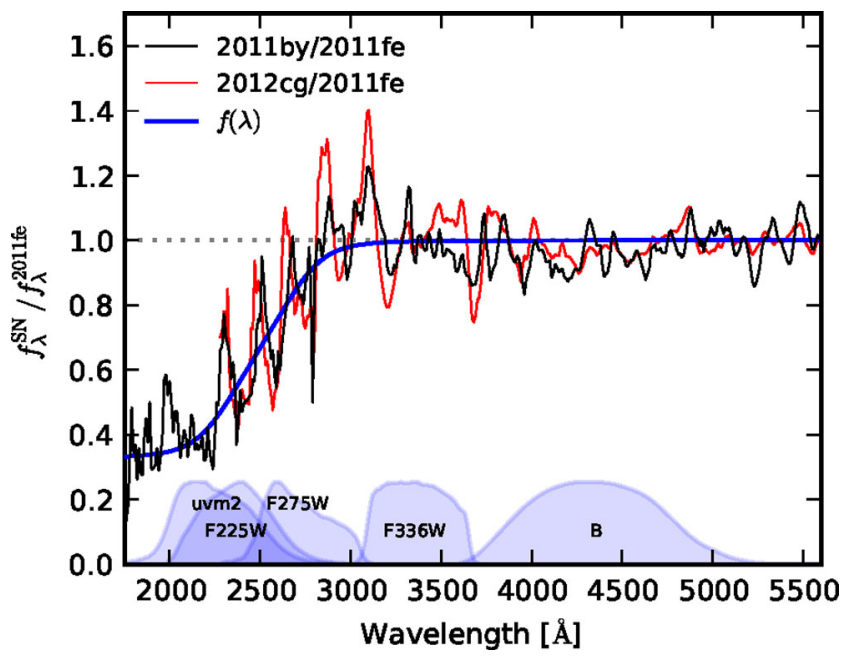

Figure 14. The flux ratio between $\mathrm{SNe} 2011$ by and $2011 \mathrm{fe}$, and $2012 \mathrm{cg}$ and $2011 \mathrm{fe}$ at maximum after they have been corrected for Galactic extinction, corrected for reddening (in the case of SN $2012 \mathrm{cg}$ ), and normalized between 5000 and $5500 \AA$ A. We use the reddening parameters derived for maximum for SN 2012cg from Table 6 and both spectra have been smoothed using boxcar smoothening. These spectra are only shown for wavelengths where the signal to noise per spectral element exceeds 2.5. Some of the passbands have been plotted in the background to illustrate which filters are affected by the difference in spectroscopic shape. The blue line shows equation (C1) that is used to model the flux ratio between SNe 2011by and $2011 \mathrm{fe}$ as described in Appendix C.

\subsection{SN 2011by as colour template}

SN 2011by was discovered (Jin \& Gao 2011) in NGC 3972 and found to be a spectroscopically normal SN Ia (Silverman, Ganeshalingam \& Filippenko 2013b) with minimal reddening (Maguire et al. 2012). HST/STIS UV observations were obtained at maximum (Programme GO-12298; PI: Ellis) and using these data Foley \& Kirshner (2013) and Graham et al. (2015a) have shown that while SN 2011 by is almost identical to SN 2011fe in its optical properties it is intrinsically fainter in the UV. The two SNe are compared at maximum in Fig. 14, where the ratio between the spectra are plotted. Comparing this to the ratio between SNe 2012cg and 2011fe at maximum, also shown in the figure, suggests that SN 2011by may be an even better reference SN than SN 2011fe in the UV.

Both SNe 2011by and 2012cg are fainter than SN 2011fe in the UV, which could explain why SN 2012cg is marginally redder in its UV colours than predicted by the fitted reddening laws. Two other $\mathrm{SNe}$ in the sample, 2012bm and 2012cp, show a similar behaviour for the bluest colour $F 225 W-V$.

HST/STIS spectroscopy of SN 2011 by was obtained at phases -10 and $-1 \mathrm{~d}$, which unfortunately does not allow us to construct an SED covering the full range of our observations as we did for SN $2011 \mathrm{fe}$. However, we can use the available spectrum at maximum and the Swift observations of this SN to model the difference between SNe 2011by and 2011fe in the UV. In Appendix C, we describe this procedure and construct a model SED of SN 2011by under the assumption that it is identical to SN $2011 \mathrm{fe}$ at wavelengths $\lambda>3000 \AA$.

Using this as the comparison $\mathrm{SN}$, we can refit the reddening laws for the SNe 2012cg, 2012cp and 2012bm for which we have reason to believe that SN 2011 by may provide a better comparison object. The fitted F99 parameters at maximum are presented in Table 8 . The fitted parameters for SNe 2012cp and 2012bm change marginally 
Table 8. Best fitted parameters of the F99 law using the colour and SED model that has been adapted to match SN 2011by in the UV as described in Section C. The quoted uncertainties are the statistical $1 \sigma$ errors from the $\chi^{2}$ fit.

\begin{tabular}{lccc}
\hline & $2012 \mathrm{cg}$ & $2012 \mathrm{cp}$ & $2012 \mathrm{bm}$ \\
\hline$E(B-V)$ & $0.12(0.02)$ & $0.34(0.03)$ & $0.45(0.04)$ \\
$R_{V}$ & $3.7_{-1.0}^{+1.4}$ & $3.2_{-0.4}^{+0.5}$ & $3.0_{-0.4}^{+0.4}$ \\
$t_{B}^{\max }$ & $56080.5(0.3)$ & $56081.1(0.3)$ & $56018.3(1.1)$ \\
$s_{B}$ & $1.13(0.02)$ & $1.19(0.02)$ & $1.24(0.08)$ \\
$\chi^{2} / v$ & 0.88 & 1.09 & 2.13 \\
\hline
\end{tabular}

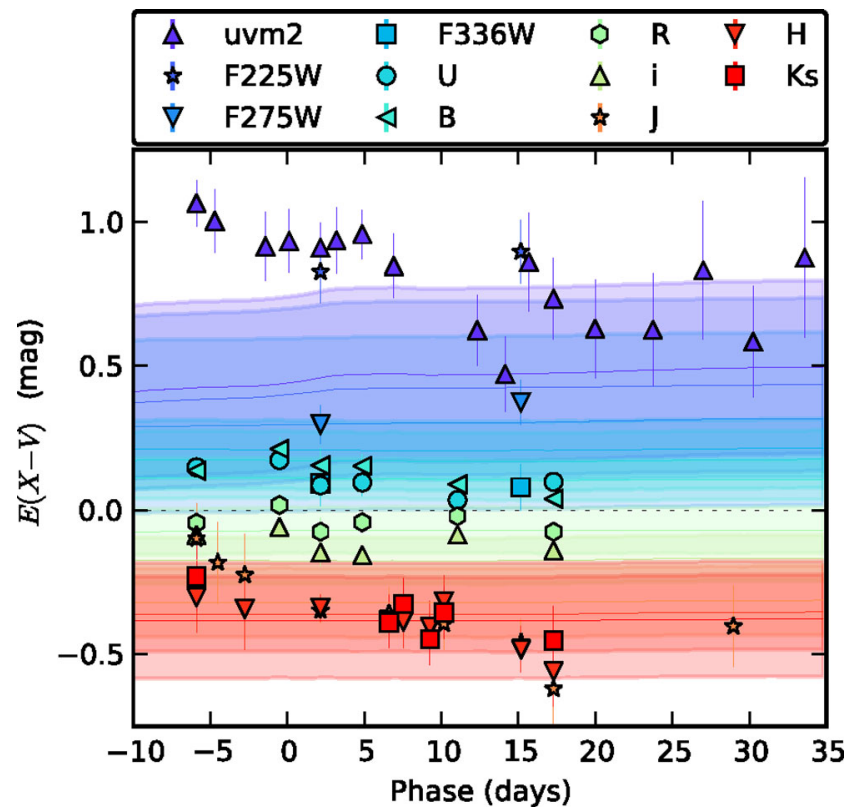

Figure 15. The colour excess for SN $2012 \mathrm{cg}$, similar to the upper-left panel of Fig. 6, with the exception that the measured and predicted colour excesses are based on the SED model described in Section C, which has been derived to mimic SN 2011 by in the UV. The solid lines represent the best fitted F99 law with the parameters given in Table 8 .

compared to Table 5 but we do note that reduced $\chi^{2}$ has decreased. However, the most interesting result here is for SN 2012cg. Not only the fitted reddening law parameters change, but also the timeevolution of $E(u v m 2-V)$ becomes less significant, which can be seen in Fig. 15. This can be explained from Fig. C1, where the colour excesses of SN 2011by are shown with respect to SN 2011fe, and a similar time-evolution for $E(u v m 2-V)$ is shown. We note that the there is still a tension between the bluest colours and the fitted reddening law which may suggest that the spectrum of SN 2012cg drops faster than SN 2011 by at $\lambda<2500 \AA$.

From the spectroscopic similarities between SNe 2011by and 2012cg as shown in Fig. 14 and the similar UV-optical colours we find that SN 2011by appears to be a better comparison SN for SN 2012cg than SN 2011fe. This study also show how studying the UV-colour evolution of SNe Ia provides a powerful tool for comparing predictions of CS dust scenarios with intrinsic colour variations.

\subsection{The impact of the UV data}

Another approach for testing the robustness of the reddening laws is to study how the fitted parameters are affected by the data at different
Table 9. The best fitted F99 parameters using either only the optical-NIR colours or all colours (UV-NIR) while the $t_{B}^{\max }$ and $s_{B}$ parameters were fixed to the values given in Tables 5 and 8 , respectively. All colour data between phases -10 and $+35 \mathrm{~d}$ from $t_{B}^{\max }$ were used for the fits.

\begin{tabular}{lcccc}
\hline SN & $\lambda$-range & $E(B-V)$ & $R_{V}$ & $\chi^{2} / v$ \\
\hline SN2012cg $^{a}$ & UV-NIR & $0.11(0.02)$ & $3.8_{-1.0}^{+1.5}$ & 0.71 \\
& OPT-NIR & $0.11(0.05)$ & $4.0_{-1.5}^{+3.2}$ & 0.56 \\
SN2012et & UV-NIR & $0.16(0.02)$ & $1.8_{-0.5}^{+0.6}$ & 1.06 \\
& OPT-NIR & $0.15(0.09)$ & $<1.9$ & 1.72 \\
SN2012cp $^{a}$ & UV-NIR & $0.34(0.03)$ & $3.1_{-0.4}^{+0.5}$ & 0.83 \\
& OPT-NIR & $0.36(0.04)$ & $3.0_{-0.5}^{+0.6}$ & 1.03 \\
SN2012bm $^{a}$ & UV-NIR & $0.45(0.03)$ & $3.0_{-0.3}^{+0.4}$ & 1.68 \\
& OPT-NIR & $0.41(0.05)$ & $3.5_{-0.5}^{+0.6}$ & 0.70 \\
SN2012cu & UV-NIR & $0.99(0.03)$ & $2.8_{-0.1}^{+0.1}$ & 0.47 \\
& OPT-NIR & $1.00(0.05)$ & $2.7_{-0.2}^{+0.2}$ & 0.43 \\
SN2014J & UV-NIR & $1.36(0.02)$ & $1.4_{-0.1}^{+0.1}$ & 2.01 \\
& OPT-NIR & $1.28(0.04)$ & $1.6_{-0.1}^{+0.1}$ & 2.29 \\
\hline
\end{tabular}

Note. ${ }^{a}$ SN colours were compared to SN 2011 by.

Table 10. The best fitted power-law parameters for the two $\mathrm{SNe}$ with low- $R_{V}$ values. The fits were carried out using either only the optical-NIR colours or all colours (UV-NIR) while the $t_{B}^{\max }$ and $s_{B}$ parameters were fixed to the values given in Table 5 . All colour data between phases -10 and $+35 \mathrm{~d}$ from $t_{B}^{\max }$ were used for the fits.

\begin{tabular}{lcccc}
\hline SN & $\lambda$-range & $A_{V}$ & $\beta$ & $\chi^{2} / \nu$ \\
\hline SN2012et & UV-NIR & $0.30(0.13)$ & $-1.7(0.5)$ & 0.63 \\
& OPT-NIR & $0.16(0.11)$ & $-3.3(1.5)$ & 0.82 \\
SN2014J & UV-NIR & $1.97(0.10)$ & $-2.0(0.1)$ & 1.40 \\
& OPT-NIR & $1.93(0.10)$ & $-2.1(0.1)$ & 1.02 \\
\hline
\end{tabular}

wavelengths. We do this by comparing the fitted F99 parameter values for two different cases, where we use all measured colours (UV-NIR) and where we omit the UV data (OPT-NIR). A tension between the two cases could be a sign of either assuming an incorrect reddening law or colour reference $\mathrm{SN}$. We keep $t_{B}^{\max }$ and $s_{B}$ fixed for this study and use SN 2011by as reference for the SNe with red UV colours. The results are presented in Table 9.

The results are consistent for all SNe except for SN 2014J, where the addition of the UV data shifts the fitted values by $\sim 2 \sigma-3 \sigma$. We fit a low value of $R_{V}$ for this $\mathrm{SN}$ and, as shown in Fig. 8, a power law appears to provide a better fit in this case. In Table 10, we present the results on the two colour sets when using a power-law relation for the two low- $R_{V} \mathrm{SNe}$. While there is a slight, $\sim 1 \sigma$, tension for SN 2012et, the results are fully consistent between the two cases for SN 2014J.

The major impact of adding the UV-colours is, however, on the uncertainties of the fitted values, in particular for low-reddening SNe. This is illustrated in Fig. 16 where the grey contour shows the 68 percent confidence region for $E(B-V)$ and $R_{V}$ when the F99 law is fitted to the optical-NIR data of SN 2012cg, while the blue contour shows the uncertainties from the full UV-NIR fit. From the figure, we conclude that extending the wavelength range significantly improves the constraints on $R_{V}$ despite the high intrinsic dispersion adopted for the Swift and HST colours. 


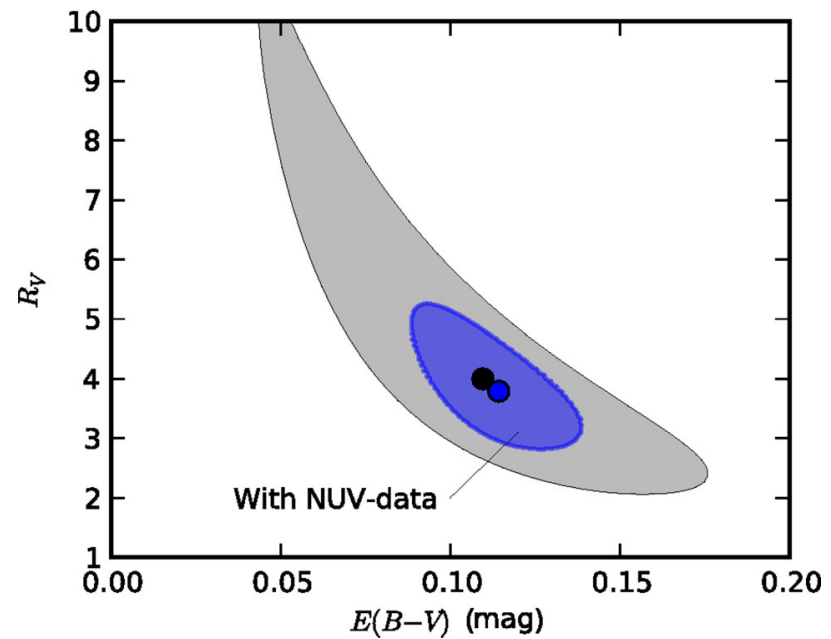

Figure 16. Contours showing the joint 68 per cent uncertainty region for the fitted parameters $R_{V}$ and $E(B-V)$ for a F99 extinction laws for SN 2012cg in comparison to SN 2011by. The two contours show the fit results when the full data set (UV-NIR) is used (dark blue contour region) and when the colours based on HST and Swift UV observations are omitted (light grey contour region). All data between phases -10 and $+35 \mathrm{~d}$ with respect to $t_{B}^{\max }$ were used, and the best-fitting values for the two cases have been marked by black and blue dots, respectively.

\subsection{Extinction from interstellar absorption features}

In Section 5, we discussed the constraints on the existence of CS dust that can be derived from non-detection of time-varying $\mathrm{Na}$ ID absorption lines. $\mathrm{Na} \mathrm{ID}$ lines in high-resolution spectra are also commonly used as a proxy for reddening (Poznanski et al. 2011) and the equivalent width of the lines have been shown to correlate well with extinction in the Milky May (see Munari \& Zwitter 1997; Poznanski et al. 2012). However, Phillips et al. (2013) found that of their sample of SNe Ia $\sim 25$ per cent, of which most have blue shifted $\mathrm{Na}$ ID features, have unusually high $\mathrm{Na}$ I column densities compared to the values expected from the Poznanski et al. (2012) relation. It is currently unclear whether the high abundance of sodium is intrinsic to the environment of some SN Ia or if the Milky Way reference value is anomalous compared to other galaxies. Other species, such as diffuse interstellar bands, have been shown to be useful for studying the ISM in SNe Ia host galaxies (Sollerman et al. 2005) and in particular the diffuse interstellar band (DIB) feature at $5780 \AA$ may provide a better proxy for reddening of SNe Ia than $\mathrm{Na}$ ID (Phillips et al. 2013). In Table 11, we present equivalent width values of $\mathrm{Na}$ ID and the DIB at $5780 \AA$ with corresponding reddening estimated by the empirical relations (Poznanski et al. 2012) and (Phillips et al. 2013), respectively, for the SNe of which we have obtained spectra or published data exists. Further empirical reddening relations exist for ISM features $\mathrm{Ca}$ II $\mathrm{H} \& \mathrm{~K}$ (Murga et al. 2015) and Mg II (Ménard et al. 2008). These relations have so far not been extensively studied for their validity in estimating the reddening of $\mathrm{SNe}$.

Besides $\mathrm{Na} I \mathrm{D}$, narrow $\mathrm{Ca}$ II $\mathrm{H} \& \mathrm{~K}$ features, with respective equivalent widths of $770 \pm 30$ and $579 \pm 35 \mathrm{~m} \AA$ could only be measured from the first FIES epoch of SN $2012 \mathrm{cg}$. These values are well above the range in which the relations in (Murga et al. 2015) are valid. Phillips et al. (2013) also quote the detection of the DIB $5780 \AA$ which is consistent with the non-detection in the individual FIES epochs within the signal to noise. The most prominent absorption features in $\mathrm{Na} \mathrm{ID}$, shown in Fig. 10 roughly agree with $v_{h}$ from Table 1 and are slightly blueshifted with respect to the apparent stellar velocity of $\sim 448 \mathrm{~km} \mathrm{~s}^{-1}$ along the line of sight of SN $2012 \mathrm{cg}$ (see Cortés, Kenney \& Hardy 2006). Additional features of the $\mathrm{Na}$ ID and $\mathrm{Ca}$ II H\&K profiles span a range from $\sim 370-530 \mathrm{~km} \mathrm{~s}^{-1}$, whereby a number of features are blended on the redshifted side of the profile. SN 2012cg is included in the analysis of Phillips et al. (2013) and is not counted in the group of SNe with anomalously high $\mathrm{Na}$ I column densities. However, the best fit $E(B-V)$ in Table 9 for $2012 \mathrm{cg}$ highly disagrees with the value computed from the Poznanski et al. (2012) relation.

Four epochs of high-resolution spectra of SN 2012cu have been described in Sternberg et al. (2014). No time-variability is detected in the deep $\mathrm{Na}$ ID absorption features, which likely consist of several blended components. The velocity of the Na ID lines of $\sim 1130 \mathrm{~km} \mathrm{~s}^{-1}$ are in agreement with the $\mathrm{H}$ I velocity along the line of sight of SN 2012cu in NGC 4772 (Haynes et al. 2000). We perform the photoionization analysis described in Section 5 on the $\mathrm{Na}$ ID doublet of 2012cu. The non-variation of Na ID1 indicates that there is no $\mathrm{Na}$ I between $6 \times 10^{18}-2 \times 10^{19} \mathrm{~cm}$. However, these constraints are weak since the absorption lines are close to saturation and not optically thin. The $E(B-V)$ computed from the Poznanski et al. (2012) relation is $\sim 2 \sigma$ greater than the photometric value. Furthermore, this value must be considered a lower limit since the $\mathrm{Na}$ ID doublet of SN 2012cu appears to be close to saturation.

SN 2012et is included in the sample of Maguire et al. (2013) in which an Na ID2 equivalent width is quoted from a mid-resolution spectrum. The corresponding Poznanski et al. (2012) relation $E(B-V)$ is $\sim 1 \sigma$ greater than expected.

Table 11. Equivalent width values of Na ID, the DIB feature at $\lambda 5780 \AA$ and $\mathrm{Mg}$ II at $\lambda 2796 \AA$ for which reddening parameters can be inferred from Poznanski et al. (2012), Phillips et al. (2013) and Ménard et al. (2008), respectively. Upper limits are determined for Mg II measurements, since the doublet is blended in the STIS spectra. In the case of SN 2014J, the Na ID equivalent width extrapolates the relation by Poznanski et al. (2012) beyond any reasonable value for $E(B-V)$. Best photometric $E(B-V)$ values have been taken from Table 9 for comparison and the $E(B-V)$ for $2011 \mathrm{fe}$ and 2011 by is assumed to be negligible.

\begin{tabular}{|c|c|c|c|c|c|c|c|c|}
\hline $\mathrm{SN}$ & $\begin{array}{c}\mathrm{Na} \text { ID1 } \\
(\mathrm{m \AA})\end{array}$ & $\begin{array}{c}\mathrm{Na} \text { ID2 } \\
(\mathrm{m \AA})\end{array}$ & $\begin{array}{c}E(B-V) \\
(\mathrm{Na} \mathrm{ID})\end{array}$ & $\begin{array}{c}\text { DIB } 5780 \\
(\mathrm{m \AA})\end{array}$ & $\begin{array}{c}A_{V} \\
(\mathrm{DIB})\end{array}$ & $\begin{array}{c}\text { Mg II } 2796 \\
(\AA)\end{array}$ & $\begin{array}{c}E(B-V) \\
(\mathrm{Mg} \mathrm{II})\end{array}$ & $\begin{array}{c}E(B-V) \\
\text { (Phot.) }\end{array}$ \\
\hline $2014 J$ & $2558(6)$ & $2831(9)$ & - & 344 (4) & $1.8(0.9)$ & $\leq 4.6(0.2)$ & $\leq 0.14(0.08)$ & $1.36(0.02)$ \\
\hline $2012 \mathrm{cg}$ & $700(9)$ & $1018(8)$ & $1.6_{-0.3}^{+0.4}$ & $85(5)^{a}$ & $0.4(0.2)$ & $\leq 3.5(0.2)$ & $\leq 0.08(0.05)$ & $0.11(0.02)$ \\
\hline $2012 \mathrm{cu}$ & $849(3)^{b}$ & $925(3)^{b}$ & $1.7_{-0.3}^{+0.4}$ & - & - & - & - & $0.99(0.03)$ \\
\hline 2012et & - & $650(40)^{c}$ & $0.3_{-0.1}^{+0.3}$ & - & - & - & - & $0.16(0.02)$ \\
\hline $2011 \mathrm{fe}$ & $27.4(1.1)^{d}$ & $47.1(0.8)^{d}$ & $0.017_{-0.003}^{+0.004}$ & - & - & $2.9(0.2)$ & $0.06_{-0.02}^{+0.03}$ & - \\
\hline 2011by & - & - & - & - & - & $\leq 2.3(0.2)$ & $\leq 0.04(0.02)$ & - \\
\hline
\end{tabular}

Note. Equivalent width values retrieved from ${ }^{a}$ Phillips et al. (2013), ${ }^{b}$ Sternberg et al. (2014), ${ }^{c}$ Maguire et al. (2013) and ${ }^{d}$ Patat et al. (2013). 


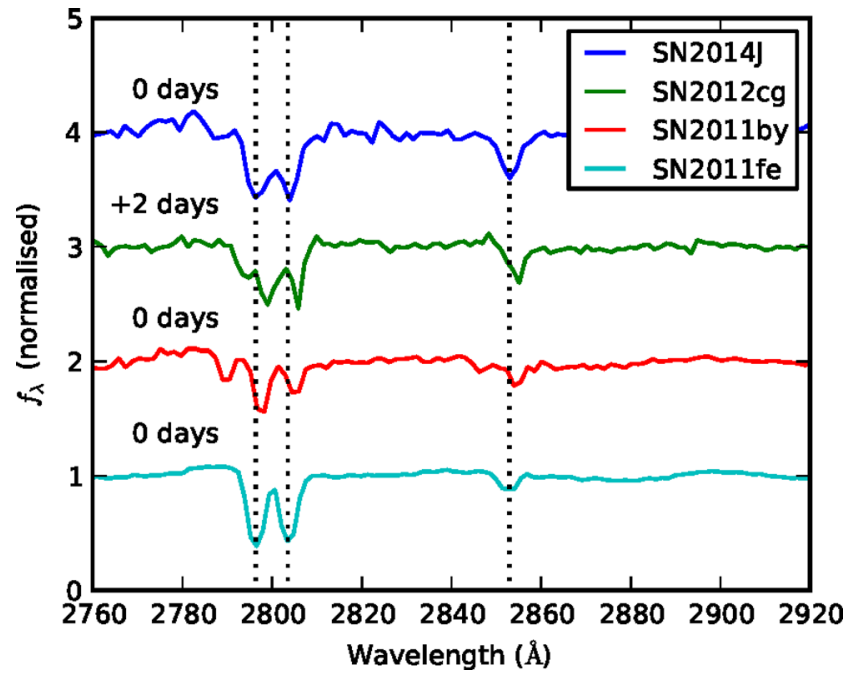

Figure 17. $\mathrm{Mg}$ II at $\lambda 2796,2803 \AA$ and $\mathrm{Mg}$ I at $\lambda 2853 \AA$ marked by dotted lines in STIS spectra of four SNe with different reddening. The continua of the unsmoothed spectra are normalized and offset for clarity. Above the respective spectra, the phases are indicated at which the spectra were taken.

It is clear from Phillips et al. (2013) that $\mathrm{Na}$ ID do not serve as good reddening proxies for SNe Ia and that the scatter in the Poznanski et al. (2012) relation is underestimated. This appears to be the case for SNe with high reddening, such as 2014J, as well as low reddening such as $2012 \mathrm{cg}$. Furthermore, many of the Na ID profiles do not appear to be optically thin, implying that the column densities are rather underestimated, which would further increase the discrepancy between the Poznanski et al. (2012) relation and observations. There is thus motivation to investigate other ISM species, such as e.g. Mg II, which are more difficult to observe and have not been studied in large samples so far.

STIS spectra of SNe 2014J (from Foley et al. 2014), 2012cg, $2011 \mathrm{fe}$ and $2011 \mathrm{by}^{7}$ around the $\mathrm{Mg}$ II doublet at $\lambda 2796,2803 \AA$ are shown in Fig. 17. The absorption features can be used to test the relation

$E(B-V)=(0.008 \pm 0.001) W^{(1.88 \pm 0.17)}$

of Ménard et al. (2008), where $W$ is the equivalent width of $\mathrm{Mg}$ II at $\lambda 2796 \AA$. The STIS spectra of SNe 2012bl, 2012bm, 2012cp and 2012 et are not included in the sample due to the low signal to noise around the $\mathrm{Mg}$ II doublet. Upon visual inspection of Fig. 17, one can see that the $\mathrm{Mg}$ II lines are deep for all four $\mathrm{SNe}$ and the line ratios obtained from fitting a double Gaussian profile further indicate that the lines are not optically thin. In all STIS spectra, except those of $2011 \mathrm{fe}$, the $\mathrm{Mg}$ II doublet is strongly blended. Further, it cannot be excluded that the entire absorption is attributed to the Milky Way. We therefore measure the total equivalent width of the observed features and consider $2 / 3$ of the value to be the upper limit of the component at $\lambda 2796 \AA$. The fraction is chosen from the line ratio of the $\mathrm{Mg}$ II doublet, if it is optically thin. The limits we obtain along with the corresponding $E(B-V)$ computed from the relation above are shown in Table 11. The $\mathrm{Mg}$ II doublet is resolved better in the 2011fe STIS spectra and the equivalent width can be determined from the Gaussian fits more accurately. The value presented in Table 11 is the average equivalent width computed from

${ }^{7}$ STIS spectra of SNe $2011 \mathrm{fe}$ and 2011 by were taken as part of Programme GO-12298; PI: Ellis. all available $2011 \mathrm{fe}$ STIS spectra. In comparison to the photometric $E(B-V)$, the Ménard et al. (2008) relation clearly underestimates the reddening of 2014J, whereas no firm conclusions can be drawn for the other SNe due to the large errors and since blending with Milky Way features cannot be excluded.

\subsection{The origin of $\mathrm{SN}$ reddening}

While it can be expected that the observed SN Ia colour arise from a mixture of extinction by dust and intrinsic SN Ia colour diversity, the latter is typically of order $|\Delta(B-V)| \lesssim 0.1$ (e.g. N08). Thus, we can assume that the impact of dimming by dust will dominate with increased observed reddening and that the fitted extinction laws will be less susceptible to intrinsic SN Ia variations. It is therefore particularly interesting to compare the fits for SNe 2012cu and 2014J. They are the reddest objects in our sample, and at the same time, show very different total-to-selective extinction, $R_{V}=2.8 \pm 0.1$ and $R_{V}=1.4 \pm 0.1$ for SNe 2012cu and 2014J, respectively.

The evidence for material in the line of sight is strengthened by the fact that both SNe show deep Na ID absorption lines in their spectra (e.g. Sternberg et al. 2014; Goobar et al. 2014, for 2012cu and 2014J, respectively). Since CS dust is expected to give rise to steep extinction laws, such a scenario may offer an explanation for the low $R_{V}$ of SN 2014J. However, no time-variability has been found for the $\mathrm{Na} I \mathrm{D}$ absorption lines for any of the SNe we studied, although Graham et al. (2015b) report a detection of time-varying $\mathrm{K}$ I absorption for SN 2014J. When combined with the non-varying $\mathrm{Na}$ ID, these findings point to CS dust at radii of $r_{\text {dust }} \gtrsim 10^{19} \mathrm{~cm}$ due to the difference in ionization energies. The detection of light echoes (Crotts 2015) also points to the existence of dust at a comparable distance from the site of the explosion, 11 pc, i.e. $r_{\text {dust }}=3 \times 10^{19} \mathrm{~cm}$.

Further, we have studied the PSF shape of SN 2014J in the HST A14 photometry to search for signs of dust at smaller radii but none could be detected, which is consistent with the lack of timeevolution of the SN 2014J colours with respect to SN 2011fe. For CS dust at $r_{\text {dust }} \gtrsim 10^{19} \mathrm{~cm}$ the photon time-delay exceeds the typical time-scale of the SN Ia light curve and no colour time-evolution is expected as discussed in Amanullah \& Goobar (2011).

Other observations suggesting that the nature of the extinction of SN 2014J is primarily interstellar, is that the polarization angle of the SN light is well aligned with the spiral structure of the host galaxy (Patat et al. 2015), the lack of thermal emission from the SN environment in near and mid-IR (Johansson et al. 2014), and that the velocities of the observed multiple $\mathrm{Na}$ ID lines are consistent with measured $\mathrm{H}$ I velocities along the line of sight in the host galaxy (Ritchey et al. 2015). The latter is also the case for SNe 2012cu (Haynes et al. 2000). Even if dust does exist at $r_{\text {dust }} \sim 10^{19} \mathrm{~cm}$ this is not expected to have any major impact on the measured $R_{V}$ for such large distances (Wang 2005).

If the reddening of both SNe 2012cu and 2014J are dominated by interstellar extinction, the dust properties along the two lines of sight must be significantly different, and the dust in M82 must be of a nature that has not been observed in the Milky Way (although see Brown et al. 2015, for a possible explanation). M82 is a starburst galaxy, and the dust properties have been studied by Hutton et al. (2014). They find that a steep Milky Way-like law is preferred over the Calzetti (2001) law, used for starburst galaxies and they derive a wavelength dependence of the scattering within a central projected radius of $<3 \mathrm{kpc}$ that suggests that 'only small grains are entrained in the inner SNe-driven wind', which is consistent with both the location (see Table 1) and the characteristic of the observed reddening of SN 2014J. 
Table 12. Surface brightness (mag/arcsec) measured using a $2 \mathrm{kpc}$ radius centred on the $\mathrm{SN}$ position in the GALEX FUV and NUV filters.

\begin{tabular}{lcc}
\hline SN & FUV & NUV \\
\hline $2014 \mathrm{~J}$ & $26.5(0.2)$ & $24.9(0.1)$ \\
$2012 \mathrm{bm}$ & $26.7(6.1)$ & $25.9(2.4)$ \\
$2012 \mathrm{cg}$ & $25.1(0.4)$ & $24.4(0.2)$ \\
$2012 \mathrm{cp}$ & $24.7(1.3)$ & $24.6(0.7)$ \\
$2012 \mathrm{cu}$ & $26.6(2.3)$ & $26.0(1.0)$ \\
$2012 \mathrm{et}$ & $24.9(2.6)$ & $24.4(1.2)$ \\
$2012 \mathrm{bl}$ & $26.1(3.3)$ & $26.2(2.1)$
\end{tabular}

For SNe Ia, Rigault et al. (2013) found that when using $\mathrm{H} \alpha$ emission as a tracer for ongoing star formation, SNe with local $\mathrm{H} \alpha$ emission is on average redder and more homogeneous, resulting in a lower brightness dispersion. These results have been confirmed by Kelly et al. (2015) and Rigault et al. (2015) using UV data from the Galaxy Evolution Explorer (GALEX). If the low $R_{V}$ originates from small dust grains in regions undergoing intense star formation, we can expect to observe different local properties between $\mathrm{SNe} 2012 \mathrm{cu}$ and 2014J. In Table 12, we present the surface brightness of at the position of the $\mathrm{SNe}$ using an aperture with a $2 \mathrm{kpc}$ radius. From this we cannot draw any firm conclusion based on the local UV surface brightness of the host galaxies. Even after we attempt to correct for host galaxy dust, following the recipe used in Rigault et al. (2015), we see no correlation with UV surface brightness and measured $R_{V}$.

In Fig. 18, we show the fitted F99 parameters from Table 5 for all $\mathrm{SNe}$ together with similar measurements from the literature. Low values of $R_{V}$ are not unique for $\mathrm{SNe} \mathrm{Ia}$, and has also been observed in the line of sight of other light sources such as gammaray burst afterglows (Fynbo et al. 2014) and quasi-stellar objects

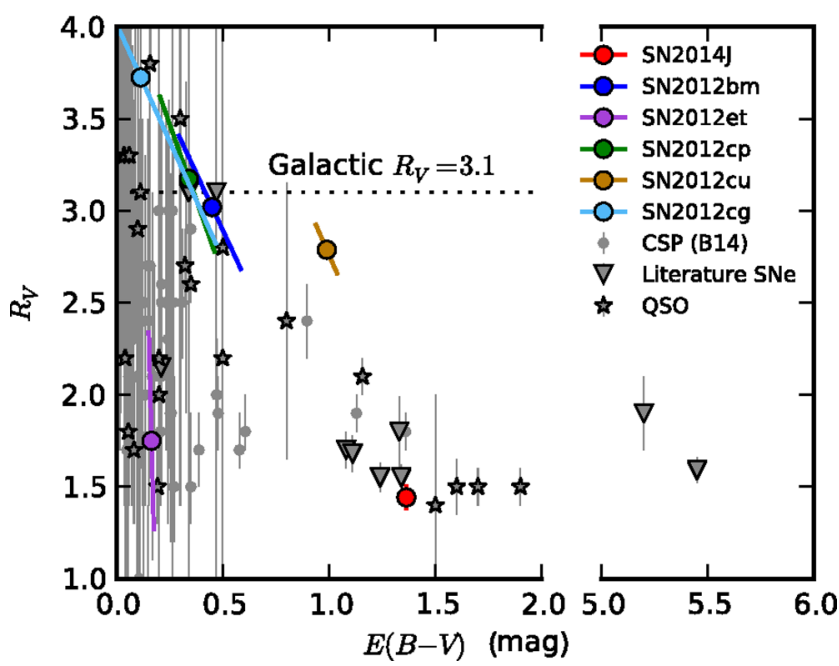

Figure 18. Fitted $R_{V}$ and $E(B-V)$ for the full phase range of -10 to $+35 \mathrm{~d}$, where SN 2011 by has been used as reference for SNe 2012bm, 2012cg, and 2012cp and SN 2011fe was used for the remaining SNe. Also plotted are measurements from the literature, where nine SNe Ia from Hernandez et al. (2000), Krisciunas et al. (2006), Krisciunas et al. (2007), Benetti et al. (2004), Elias-Rosa et al. (2006), Elias-Rosa et al. (2008), Hicken et al. (2009) and Folatelli et al. (2010) together with three Type II SNe from Pozzo et al. (2006) and Poznanski et al. (2009) are included. The parameters measured by studying reddening of QSO by foreground galaxies are from Elíasdóttir et al. (2006) and Östman, Goobar \& Mörtsell (2006).
(QSO; e.g. Fynbo et al. 2013; Leighly et al. 2014). From the figure, it is apparent that there are several observations with low- $R_{V}$ values for high-extinction SNe Ia, while SN 2012cu is both showing high extinction and a relatively high $R_{V}$. For low-extinction $\mathrm{SNe}$ it is by nature difficult to measure $R_{V}$ for individual objects, but there are several statistical studies that obtain higher $\left(R_{V} \lesssim 3\right)$ average values of $R_{V}$ compared to what is observed for high-extinction $\mathrm{SNe}$ Ia (e.g. Chotard et al. 2011; Mandel, Narayan \& Kirshner 2011). The UV observations allow us to improve the sensitivity on $R_{V}$ for individual low-reddened $\mathrm{SNe}$, and it is interesting to note that we measure $R_{V}$ discrepancies similar to what we saw for high-extinction objects. The colours of SNe 2012cg and 2012et are however a mixture of intrinsic variation as was shown by the comparison of SN 2012cg with SNe 2011by and 2011fe in particular. For these objects, we therefore cannot draw any firm conclusions to what extent the measured $R_{V}$ is solely a dust property. Note though that there are strong indications of dust along the line of sight for these SNe from Na ID absorption (as we have seen for SN 2012cg and for SN 2012et in Maguire et al. 2013). Independent of the origin of the reddening we conclude that our sample of reddened $\mathrm{SNe}$ Ia with well observed colours, although limited, suggests that a range of $R_{V}$ values can be expected for both low and high-reddened $\mathrm{SNe}$ Ia.

Given the findings of M13 that all 'NUV-blue' SNe belong to the photospheric NV class while the 'NUV-red' consists of both NV and $\mathrm{HV} \mathrm{SNe}$ and the colour-velocity relation for $B-V$ (Wang et al. 2009), we can also compare the derived extinction parameters with the measured Si II $\lambda 6355 \AA$ velocities. From Figs 12 and 18, we conclude that both $\mathrm{SNe}$ for which we measure a low $R_{V}$ (2014J and 2012et) are classified as HV SNe while the remaining belong to the NV class. This is consistent with the statistical study of samples of SNe Ia by Wang et al. (2009), although our sample size does not allow us to draw firm conclusions. However, the fact that we present $\mathrm{SNe}$ with both high and low values of $R_{V}$ in both high- and low-extinction environments argues against that the origin of the discrepancy in observed reddening laws is solely due to intrinsic $\mathrm{SN}$ properties.

One possible explanation to both of these findings could be that HV SNe primarily explode in environments with low $R_{V}$ dust. Wang et al. (2013) and Pan et al. (2015) have shown that HV SNe are, on average, both located in more luminous hosts, and in brighter regions, closer to their host nuclei, compared the corresponding NV samples. Further, the results from Hutton et al. (2014) showed that a steep Milky Way law is preferred for the central region of M82, and similarly the extinction towards the Galactic bulge also follows a steeper extinction curve $\left(R_{V} \approx 2.5\right.$ Nataf et al. 2013) compared to the Milky Way average. Both the dust and SN Ia properties are likely to be affected by the environment where they are produced and if HV SNe Ia originate from younger and more metal-rich progenitors than NV SNe Ia (Wang et al. 2013), it is possible that the average dust properties in the two environments are different as well.

Studies of high-redshift SNe for cosmological applications are unlikely to include highly reddened SNe like 2012cu and 2014J, but objects with similar reddening to SNe 2012cg and 2012et are common. In Fig. 19, we show the normalized colour excesses, $E(X$ $-V) / E(B-V)$, for the three least reddened SNe, where we have used the approach from Fig. 7 to obtain the plotted wavelengths. Fig. 19 also shows the average reddening F99 law and the SALT2 colour law (Guy et al. 2007b) which has been empirically derived without attempting to discriminate between the different sources of $\mathrm{SN}$ reddening. Plotted here is the version used in the cosmology analysis from Betoule et al. (2014). Although the reddening laws are 


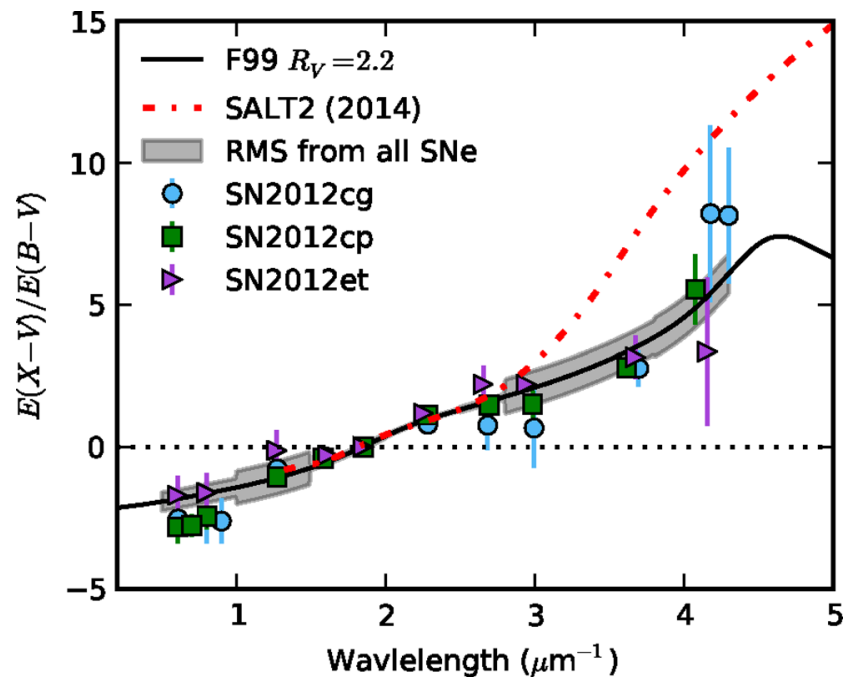

Figure 19. The normalized colour excess $E(X-V) / E(B-V)$ is shown using the same approach as in Fig. 7 for the measurements of the three least reddened $\mathrm{SNe}$. The error bars are dominated by the adopted intrinsic colour uncertainties. Overplotted is also the average F99 law (solid black) for the three SNe (fitted by comparing the colours to SN 2011fe for SN 2012et and to SN 2011by for the others) and the version of the SALT2 colour law (Guy et al. 2007b) used in Betoule et al. (2014). The SALT2 law is only defined over the wavelength range 2000-8000 $\AA$. The grey regions show the RMS of the full sample with respect to the average reddening law for different wavelength bins.

consistent at wavelengths covered by the $B-R$ bands, the SALT2 law starts to diverge from the data in the UV, wavelengths that becomes increasingly important when SNe Ia at high redshifts are studied. The lack of understanding SN reddening is one of the dominating astrophysical systematic uncertainties when SNe Ia are used for cosmology (see e.g. Amanullah et al. 2010; Sullivan et al. 2011; Suzuki et al. 2012; Betoule et al. 2014) and further UV studies are needed in order to discriminate between different sources of reddening.

\section{SUMMARY AND CONCLUSIONS}

In this work, we have presented and analysed broad-band photometry of seven SNe Ia covering the wavelength range 0.2$2 \mu \mathrm{m}$, obtained mainly from HST, Swift and the NOT.

All SNe are found to be normal with respect to their light curves and optical spectroscopic properties. The $\mathrm{SNe}$ have colour excesses in the range $E(B-V)=0.0-1.4$ mag and we have studied the reddening properties by comparing them to the normal pristine SNe Ia, 2011 fe and 2011by. In particular, we have explored three different extinction laws: O’Donnell (1994), F99, and Goobar (2008).

We have further studied the time-evolution of the fitted reddening laws and tested the consistency over a broad wavelength range. For the one SN, 2012cg, we do find indications of time-evolution of the bluest colours and we have investigated if this could be explained by CS dust. Further, we have also searched for time-varying absorption features, in particular Na ID, for SN 2012cg in multi-epoch highresolution spectroscopy. The high-resolution spectra were also used to estimate the extinction along the line of sight using the absorption of $\mathrm{Na}$ ID and $\mathrm{Mg}$ II as dust proxies. The validity of using SN $2011 \mathrm{fe}$ as a colour template was discussed and compared to the resulting reddening parameters when using SN 2011by.

We summarize the main findings as follows. (i) We observe a diversity in extinction properties characterized by a total-to-selective extinction, $R_{V}$, ranging from $R_{V} \approx 1.4$ to $R_{V} \approx 3$

(ii) The diversity between the fitted reddening laws for different $\mathrm{SNe}$ is significantly greater than the discrepancy between the laws for a single SN. Although, we do find that the empirical F99 law provides a better fit for $\mathrm{SNe}$ with $R_{V} \sim 3$ while a power law is preferred for the low- $R_{V}$ SN 2014J.

(iii) For the two reddest $\mathrm{SNe}$, both with $E(B-V)>1$ mag, we observe significantly different values of $R_{V}$ with $R_{V}=2.8 \pm 0.1$ and $R_{V}=1.4 \pm 0.1$, for SN 2012cu and 2014J, respectively. Based on their $\mathrm{Na}$ ID profiles being consistent with the observed $\mathrm{H}$ I velocity fields in their host galaxies, the lack of thermal emission and the observed polarization being aligned with the host galaxy plane for SN 2014J, we conclude that the reddening is likely dominated by extinction in the ISM of the host galaxy. Further, pre-existing CS dust at radii $r_{\text {dust }}<10^{19} \mathrm{~cm}$ is expected to give rise to evolution of the colour excesses, $E(X-V)$, but this is not observed for either SN 2012cu nor SN 2014J.

(iv) We compare nebular spectra of SNe 2011fe, 2012cg and $2014 \mathrm{~J}$ and conclude that all three are very similar at epochs $+280 \mathrm{~d}$ from maximum, with SNe $2011 \mathrm{fe}$ and $2012 \mathrm{cg}$ being remarkably similar.

(v) We compare the STIS spectra at maximum of SNe 2011by, $2011 \mathrm{fe}$ and $2012 \mathrm{cg}$ and conclude that while SN 2012cg is well matched to both SNe at $\lambda>3800 \AA$ it is better matched to SN 2011 by at shorter wavelengths.

(vi) For SN 2012cg we observe time-evolution in E(uvm $2-V)$ with respect to SN 2011fe, but when the object is compared to SN 2011 by the evolution is less significant. CS dust at $r_{\text {dust }} \sim 10^{17}$ $\mathrm{cm}$ is consistent with $E(u v m 2-V)$ but disfavoured by the lack of evolution of the redder colours. However, this example illustrates that observations over a wide wavelength range provides a powerful tool for separating the origin of colour excess evolution of SNe Ia.

(vii) For low-reddening SNe such as SN 2012cg, adding UV data to optical and near-IR can decrease the statistical uncertainty of $R_{V}$ by $>50$ per cent.

(viii) The two $\mathrm{SNe}$ with low $R_{V}$ are found to have the highest $\mathrm{Si}$ II velocities at maximum which is consistent with previous studies (Wang et al. 2009).

(ix) By measuring the equivalent width from absorption of $\mathrm{Na}$ I, we confirm the results of Phillips et al. (2013), and find that the extinction derived for SNe 2012cg, 2012cu and 2012et using the Poznanski et al. (2012) relation is higher than what we observe directly for these objects.

Future studies, including a larger sample of low-reddening objects covering a wide range in host galaxy morphologies, will be necessary to robustly estimate the impact of the diversity in the wavelength dependence of reddening on the estimates of cosmological parameters from SNe Ia. The current work, clearly showing that there is significant range in the extinction parameters, and possibly also extinction laws, should motivate further multiwavelength studies of SNe Ia, also including the UV.

\section{ACKNOWLEDGEMENTS}

We would like to thank Denise Taylor at Space Telescope Science Institute for advising and assisting us in carrying out this programme. We would also like to thank Livia Vallini for carrying out observations of SN 2012cg during the Nordic Millimetre and Optical/NIR Astronomy Summer School 2012. We are grateful 
to the anonymous referee for thoroughly going through the manuscript and providing us with many useful comments. RA and AG acknowledge support from the Swedish Research Council and the Swedish Space Board. PJB and the Swift Optical/Ultraviolet Supernova Archive (SOUSA) are supported by NASA's Astrophysics Data Analysis Program through grant NNX13AF35G. The work of PS is sponsored by FCT - Fundação para a Ciência e Tecnologia, under the grant SFRH/BD/62075/2009. VS acknowledges support from Fundação para a Ciência e a Tecnologia (Ciência 2008) and grant PTDC/CTE-AST/112582/2009. NER acknowledges the support from the European Union Seventh Framework Programme (FP7/2007-2013) under grant agreement no. 267251 'Astronomy Fellowships in Italy' (AstroFIt). The Oskar Klein Centre is funded by the Swedish Research Council. EYH acknowledge the generous support provided by the Danish Agency for Science and Technology and Innovation through a Sapere Aude Level 2 grant. The Dark Cosmology Centre is funded by the DNRF. The research leading to these results has received funding from the European Research Council under the European Union's Seventh Framework Programme (FP7/2007-2013)/ERC Grant agreement no. EGGS-278202. Observations were made with the Hubble Space Telescope; the NOT, operated by the Nordic Optical Telescope Scientific Association at the Observatorio del Roque de los Muchachos, La Palma, Spain. The data presented here were obtained in part with ALFOSC, which is provided by the Instituto de Astrofisica de Andalucia (IAA) under a joint agreement with the University of Copenhagen and NOTSA. STSDAS and PyRAF is a product of the Space Telescope Science Institute, which is operated by AURA for NASA. This research has made use of the NASA/IPAC Extragalactic Database (NED) which is operated by the Jet Propulsion Laboratory, California Institute of Technology, under contract with the National Aeronautics and Space Administration.

\section{REFERENCES}

Amanullah R., Goobar A., 2011, ApJ, 735, 20

Amanullah R. et al., 2010, ApJ, 716, 712

Amanullah R. et al., 2014, ApJ, 788, L21 (A14)

Astier P. et al., 2006, A\&A, 447, 31

Benetti S. et al., 2004, MNRAS, 348, 261

Benetti S. et al., 2005, ApJ, 623, 1011

Betoule M. et al., 2014, A\&A, 568, A22

Blondin S., Tonry J. L., 2007, ApJ, 666, 1024

Blondin S., Prieto J. L., Patat F., Challis P., Hicken M., Kirshner R. P., Matheson T., Modjaz M., 2009, ApJ, 693, 207

Blondin S. et al., 2012, AJ, 143, 126

Borkowski K. J., Blondin J. M., Reynolds S. P., 2009, ApJ, 699, L64

Bourque M., Anderson J., 2014, STScI Tech. Rep. CTE Correction for UVIS Subarrays Without Pre-Scan Bias Pixels. STScI, Baltimore, MD

Branch D. et al., 2003, AJ, 126, 1489

Branch D. et al., 2006, PASP, 118, 560

Breeveld A. A., Landsman W., Holland S. T., Roming P., Kuin N. P. M., Page M. J., 2011, in McEnery J. E., Racusin J. L., Gehrels N., eds, AIP Conf. Proc. Vol. 1358, Gamma Ray Bursts 2010. Am. Inst. Phys., New York, p. 373

Brown T., 2011, Instrument Science Report WFC3,2008-49

Brown P. J., 2014, ApJ, 796, L18

Brown P. J. et al., 2010, ApJ, 721, 1608

Brown P. J. et al., 2012, ApJ, 753, 22

Brown P. J., Breeveld A. A., Holland S., Kuin P., Pritchard T., 2014, Ap\&SS, 354,89

Brown P. J. et al., 2015, ApJ, 805, 74

Burns C. R. et al., 2011, AJ, 141, 19

Burns C. R. et al., 2014, ApJ, 789, 32 (B14)
Calzetti D., 2001, New Astron. Rev., 45, 601

Cao Y., Kasliwal M. M., McKay A., 2014, Astron. Telegram, 5786, 1

Cappellaro E., Pastorello A., Tomasella L., Benetti S., Fiaschi M., Ochner P., Turatto M., Valenti S., 2012, Cent. Bur. Electron. Telegrams, 3077, 2 Cardelli J. A., Clayton G. C., Mathis J. S., 1989, ApJ, 345, 245 (CCM)

Cenko S. B., Filippenko A. V., Silverman J. M., Gal-Yam A., Pei L., Nguyen M., Carson D., Barth A. J., 2012, Cent. Bur. Electron. Telegrams, 3111, 2

Chomiuk L. et al., 2012a, ApJ, 750, 164

Chomiuk L., Soderberg A., Simon J., Foley R., 2012b, Astron. Telegram, 4453,1

Chotard N. et al., 2011, A\&A, 529, L4

Conley A. et al., 2008, ApJ, 681, 482

Conley A. et al., 2011, ApJS, 192, 1

Contreras C. et al., 2010, AJ, 139, 519

Cortés J. R., Kenney J. D. P., Hardy E., 2006, AJ, 131, 747

Cortés J. R., Kenney J. D. P., Hardy E., 2008, ApJ, 683, 78

Cox L. et al., 2012, Cent. Bur. Electron. Telegrams, 3130, 1

Crotts A. P. S., 2015, ApJ, 804, L37

Dalcanton J. J. et al., 2009, ApJS, 183, 67

Dennefeld M., Pizzella A., Valentini M., Tomasella L., Benetti S., Cortes M., Ozel N., Rajpurohit A., 2012, Cent. Bur. Electron. Telegrams, 3226, 2

Dilday B. et al., 2012, Science, 337, 942

Draine B. T., 2003, ApJ, 598, 1017

Elias-Rosa N. et al., 2006, MNRAS, 369, 1880

Elias-Rosa N. et al., 2008, MNRAS, 384, 107

Elíasdóttir Á., Hjorth J., Toft S., Burud I., Paraficz D., 2006, ApJS, 166, 443

Filippenko A. V., Li W. D., Treffers R. R., Modjaz M., 2001, in Paczynski

B., Chen W.-P., Lemme C., eds, ASP Conf. Ser. Vol. 246, IAU Colloq.

183: Small Telescope Astronomy on Global Scales. Astron. Soc. Pac., San Francisco, p. 121

Firth R. E. et al., 2015, MNRAS, 446, 3895

Fitzpatrick E. L., 1999, PASP, 111, 63 (F99)

Folatelli G. et al., 2010, AJ, 139, 120

Folatelli G. et al., 2012, ApJ, 745, 74

Folatelli G. et al., 2013, ApJ, 773, 53

Foley R. J., Kasen D., 2011, ApJ, 729, 55

Foley R. J., Kirshner R. P., 2013, ApJ, 769, L1

Foley R. J. et al., 2014, MNRAS, 443, 2887

Förster F., González-Gaitán S., Folatelli G., Morrell N., 2013, ApJ, 772, 19

Fossey J., Cooke B., Pollack G., Wilde M., Wright T., 2014, Cent. Bur. Electron. Telegrams, 3792, 1

Fox O. D., Filippenko A. V., 2013, ApJ, 772, L6

Fox O. D. et al., 2011, ApJ, 741, 7

Friedman A. S., 2012, PhD thesis, Harvard University

Friedman A. S. et al., 2014, ApJS, preprint (arXiv:1408.0465)

Fruchter A. S. et al., 2010, Space Telescope Science Institute Calibration Workshop, p. 382

Fynbo J. P. U., Krogager J.-K., Venemans B., Noterdaeme P., Vestergaard M., Møller P., Ledoux C., Geier S., 2013, ApJS, 204, 6

Fynbo J. P. U. et al., 2014, A\&A, 572, A12

Garavini G. et al., 2007, A\&A, 470, 411

Gehrels N. et al., 2004, ApJ, 611, 1005

Goobar A., 2008, ApJ, 686, L103

Goobar A., Leibundgut B., 2011, Annu. Rev. Nucl. Part. Sci., 61, 251

Goobar A. et al., 2014, ApJ, 784, L12

Goobar A. et al., 2015, ApJ, 799, 106

Graham M. L. et al., 2015a, MNRAS, 446, 2073

Graham M. L. et al., 2015b, ApJ, 801, 136

Graur O., Maoz D., 2012, Astron. Telegram, 4226, 1

Guy J. et al., 2007a, A\&A, 466, 11

Guy J. et al., 2007b, A\&A, 470, 411

Hamuy M. et al., 2006, PASP, 118, 2

Harutyunyan A. H. et al., 2008, A\&A, 488, 383

Haynes M. P., Jore K. P., Barrett E. A., Broeils A. H., Murray B. M., 2000, AJ, 120, 703

Hernandez M. et al., 2000, MNRAS, 319, 223 
Hicken M. et al., 2009, ApJ, 700, 331

Hsiao E. Y., Conley A., Howell D. A., Sullivan M., Pritchet C. J., Carlberg R. G., Nugent P. E., Phillips M. M., 2007, ApJ, 663, 1187 (H07)

Hsiao E. Y. et al., 2013, ApJ, 766, 72

Hutton S., Ferreras I., Wu K., Kuin P., Breeveld A., Yershov V., Cropper M., Page M., 2014, MNRAS, 440, 150

Itagaki K. et al., 2012, Cent. Bur. Electron. Telegrams, 3146, 1

Jha S., Riess A. G., Kirshner R. P., 2007, ApJ, 659, 122

Jin Z., Gao X., 2011, Cent. Bur. Electron. Telegrams, 2708, 1

Johansson J., Amanullah R., Goobar A., 2013, MNRAS, 431, L43

Johansson J. et al., 2014, MNRAS, preprint (arXiv:1411.3332)

Kandrashoff M. et al., 2012, Cent. Bur. Electron. Telegrams, 3111, 1

Kelly P. L., Filippenko A. V., Burke D. L., Hicken M., Ganeshalingam M., Zheng W., 2015, Science, 347, 1459

Kirshner R. P. et al., 1993, ApJ, 415, 589

Krisciunas K. et al., 2003, AJ, 125, 166

Krisciunas K., Prieto J. L., Garnavich P. M., Riley J.-L. G., Rest A., Stubbs C., McMillan R., 2006, AJ, 131, 1639

Krisciunas K. et al., 2007, AJ, 133, 58

Landolt A. U., 1992, AJ, 104, 340

Leibundgut B., 1988, PhD thesis, Univ. Basel

Leighly K. M., Terndrup D. M., Baron E., Lucy A. B., Dietrich M., Gallagher S. C., 2014, ApJ, 788, 123

McClelland C. M., Garnavich P. M., Milne P. A., Shappee B. J., Pogge R. W., 2013, ApJ, 767, 119

Maeda K. et al., 2010a, Nature, 466, 82

Maeda K., Taubenberger S., Sollerman J., Mazzali P. A., Leloudas G., Nomoto K., Motohara K., 2010b, ApJ, 708, 1703

Maeda K., Nozawa T., Motohara K., 2014, MNRAS, preprint (arXiv:1411.3778)

Maguire K. et al., 2012, MNRAS, 426, 2359

Maguire K. et al., 2013, MNRAS, 436, 222

Mandel K. S., Narayan G., Kirshner R. P., 2011, ApJ, 731, 120

Mandel K. S., Foley R. J., Kirshner R. P., 2014, ApJ, 797, 75

Marion G. H., Milisavljevic D., Rines K., Wilhelmy S., 2012, Cent. Bur. Electron. Telegrams, 3146, 2

Marion G. H. et al., 2015, ApJ, 798, 39

Matheson T. et al., 2012, ApJ, 754, 19

Mazzali P. A. et al., 2014, MNRAS, 439, 1959

Meikle W. P. S., 2000, MNRAS, 314, 782

Ménard B., Nestor D., Turnshek D., Quider A., Richards G., Chelouche D., Rao S., 2008, MNRAS, 385, 1053

Milisavljevic D., 2012, Cent. Bur. Electron. Telegrams, 3130, 3

Milne P. A. et al., 2010, ApJ, 721, 1627

Milne P. A., Brown P. J., Roming P. W. A., Bufano F., Gehrels N., 2013, ApJ, 779, 23 (M13)

Munari U., Zwitter T., 1997, A\&A, 318, 269

Munari U., Henden A., Belligoli R., Castellani F., Cherini G., Righetti G. L., Vagnozzi A., 2013, New Astron., 20, 30

Murga M., Zhu G., Ménard B., Lan T.-W., 2015, MNRAS, 452, 511

Nataf D. M. et al., 2013, ApJ, 769, 88

Nielsen M. T. B., Voss R., Nelemans G., 2013, MNRAS, 435, 187

Nobili S., Goobar A., 2008, A\&A, 487, 19 (N08)

Nordin J. et al., 2011, A\&A, 526, A119

Nugent P., Kim A., Perlmutter S., 2002, PASP, 114, 803

Nugent P. E. et al., 2011, Nature, 480, 344

O’Donnell J. E., 1994, ApJ, 422, 158

Ofek E. O. et al., 2012, PASP, 124, 62

Östman L., Goobar A., Mörtsell E., 2006, A\&A, 450, 971

Pan Y.-C., Sullivan M., Maguire K., Gal-Yam A., Hook I. M., Howell D. A., Nugent P. E., Mazzali P. A., 2015, MNRAS, 446, 354

Parnovsky S. L., Parnowski A. S., 2010, Ap\&SS, 325, 163

Patat F. et al., 2007, Science, 317, 924

Patat F., Cox N. L. J., Parrent J., Branch D., 2010, A\&A, 514, A78

Patat F. et al., 2013, A\&A, 549, A62

Patat F. et al., 2015, A\&A, 577, A53
Pereira R. et al., 2013, A\&A, 554, A27

Perlmutter S. et al., 1997, ApJ, 483, 565

Perlmutter S. et al., 1999, ApJ, 517, 565

Persson S. E., Murphy D. C., Krzeminski W., Roth M., Rieke M. J., 1998, AJ, 116, 2475

Phillips M. M., 1993, ApJ, 413, L105

Phillips M. M. et al., 2013, ApJ, 779, 38

Pignata G. et al., 2012, Cent. Bur. Electron. Telegrams, 3076, 1

Poznanski D. et al., 2009, ApJ, 694, 1067

Poznanski D., Ganeshalingam M., Silverman J. M., Filippenko A. V., 2011, MNRAS, 415, L81

Poznanski D., Prochaska J. X., Bloom J. S., 2012, MNRAS, 426, 1465

Pozzo M. et al., 2006, MNRAS, 368, 1169

Prieto J. L., 2012, Cent. Bur. Electron. Telegrams, 3076, 2

Puckett T. et al., 2012, Cent. Bur. Electron. Telegrams, 3077, 1

Rau A. et al., 2009, PASP, 121, 1334

Rich D. et al., 2012, Cent. Bur. Electron. Telegrams, 3226, 1

Riess A. G., Press W. H., Kirshner R. P., 1996, ApJ, 473, 88

Riess A. G. et al., 1998, AJ, 116, 1009

Rigault M. et al., 2013, A\&A, 560, A66

Rigault M. et al., 2015, ApJ, 802, 20

Ritchey A. M., Welty D. E., Dahlstrom J. A., York D. G., 2015, ApJ, 799, 197

Roming P. W. A. et al., 2005, Space Sci. Rev., 120, 95

Schlafly E. F., Finkbeiner D. P., 2011, ApJ, 737, 103

Schlegel D. J., Finkbeiner D. P., Davis M., 1998, ApJ, 500, 525

Scolnic D. M., Riess A. G., Foley R. J., Rest A., Rodney S. A., Brout D. J., Jones D. O., 2014, ApJ, 780, 37

Silverman J. M., Filippenko A. V., 2012, MNRAS, 425, 1917

Silverman J. M., Kong J. J., Filippenko A. V., 2012a, MNRAS, 425, 1819

Silverman J. M. et al., 2012b, ApJ, 756, L7

Silverman J. M. et al., 2013a, ApJS, 207, 3

Silverman J. M., Ganeshalingam M., Filippenko A. V., 2013b, MNRAS, 430, 1030

Simon J. D. et al., 2009, ApJ, 702, 1157

Smith J. A. et al., 2002, AJ, 123, 2121

Soker N., 2014, MNRAS, 444, L73

Sollerman J., Cox N., Mattila S., Ehrenfreund P., Kaper L., Leibundgut B., Lundqvist P., 2005, A\&A, 429, 559

Springob C. M., Masters K. L., Haynes M. P., Giovanelli R., Marinoni C., 2009, ApJS, 182, 474

Stanishev V. et al., 2015, A\&A, preprint (arXiv:1505.07707)

Sternberg A. et al., 2011, Science, 333, 856

Sternberg A. et al., 2014, MNRAS, 443, 1849

Stetson P. B., 1987, PASP, 99, 191

Stritzinger M. et al., 2002, AJ, 124, 2100

Sullivan M. et al., 2011, ApJ, 737, 102

Suntzeff N. B., 2000, in Holt S. S., Zhang W. W., eds, AIP Conf. Proc. Vol. 522, Cosmic Explosions: Tenth Astrophysics Conference. Am. Inst. Phys., New York, p. 65

Suzuki N. et al., 2012, ApJ, 746, 85

Taddia F. et al., 2012, A\&A, 545, L7

Telting J. H. et al., 2014, Astron. Nachr., 335, 41

Thomas R. C. et al., 2011, ApJ, 743, 27

Tomasella L. et al., 2014, Astron. Nachr., 335, 841

Tripp R., 1998, A\&A, 331, 815

Tully R. B., Rizzi L., Shaya E. J., Courtois H. M., Makarov D. I., Jacobs B. A., 2009, AJ, 138, 323

Valenti S. et al., 2011, MNRAS, 416, 3138

Wang L., 2005, ApJ, 635, L33

Wang X. et al., 2009, ApJ, 699, L139

Wang X. et al., 2012, ApJ, 749, 126

Wang X., Wang L., Filippenko A. V., Zhang T., Zhao X., 2013, Science, 340,170

Zhang T.-M., Wu C.-J., Wang X.-F., 2012, Cent. Bur. Electron. Telegrams, 3130,2 


\section{SUPPORTING INFORMATION}

Additional Supporting Information may be found in the online version of this article:

Table 3. The photometry of all SNe (http://mnras.oxfordjournals. org/lookup/suppl/doi:10.1093/mnras/stv1505/-/DC1).

Please note: Oxford University Press is not responsible for the content or functionality of any supporting materials supplied by the authors. Any queries (other than missing material) should be directed to the corresponding author for the paper.

\section{APPENDIX A: SUMMARY OF SNE STUDIED IN THIS WORK}

The seven SNe Ia that we study in this work are briefly summarized below.

(i) SN 2012bl was discovered by the Chilean Automatic Supernova Search 42.9 arcsec east and 14.6 arcsec north of the centre of the galaxy ESO 234-19 on March 26.38 uT from an unfiltered image (Pignata et al. 2012). A spectrum (Prieto 2012) was obtained with the $2.5 \mathrm{~m} \mathrm{du}$ Pont telescope at the Las Campanas Observatory on March 27.28 uTand object was classified as an SN Ia using SNID (Blondin \& Tonry 2007).

(ii) SN 2012bm, located 10.95 arcsec west and 10.4 arcsec north of the centre of the galaxy UGC 8189, was discovered on March 27 by Puckett et al. (2012). A spectrum was obtained on Mar. 28.05 UT and it was classified (Cappellaro et al. 2012) as an SN Ia using GELATO (Harutyunyan et al. 2008).

(iii) SN 2012cg was discovered (Kandrashoff et al. 2012) by the Lick Observatory Supernova Search (Filippenko et al. 2001) on May 17.220 UT. The redder than normal SN was located 17.3 arcsec east and 1.5 arcsec south (Cenko et al. 2012) of the centre of the Virgo Cluster member NGC 4424 in a region with many blue stars and disturbed dust lanes. Graur \& Maoz (2012) used pre-explosion HST images of this well-studied galaxy to rule-out most supergiants as possible binary companions for the progenitor scenario.

(iv) $\mathrm{SN} 2012 \mathrm{cp}$ was discovered 6.7 arcsec east and 1.2 arcsec south of the centre of UGC 8713 on May 23.2 uT by the Puckett Observatory Supernova Search (Cox et al. 2012). Milisavljevic (2012) and Zhang, Wu \& Wang (2012) obtained spectroscopy of the object on May 25.3 and 25.7 uT, respectively, and reported that it was consistent with an $\mathrm{SN}$ Ia before maximum.

(v) SN 2012cu was discovered on June 14.6 ut by Itagaki et al. (2012) 3.1 arcsec east and 27.1 arcsec south of the nucleus of the galaxy NGC 4772 and later classified as an SN Ia by Marion et al. (2012). Pre-explosion Chandra observations are available of the host galaxy and were used by Nielsen, Voss \& Nelemans (2013) to constrain the $\mathrm{X}$-ray emission from the progenitor scenario.

(vi) SN 2012et, located 5.3 arcsec east and 0.8 arcsec north of the centre of MCG +04-55-47, was discovered by Rich et al. (2012) on September 12.057 UT, and later classified on September 13.80 UT as a high-velocity SNe Ia (Dennefeld et al. 2012).

(vii) SN 2014J was discovered by Fossey et al. (2014) in the nearby galaxy M82 on January 21.805 uT, and later classified by Cao, Kasliwal \& McKay (2014). The SN was located 54 arcsec west and 21 arcsec south of the ill-defined nucleus of the host galaxy.

\section{APPENDIX B: A SPECTRAL AND COLOUR MODEL FROM SN 2011fe}

SN 2011fe is a normal SN Ia with excellent temporal and wavelength coverage with negligible extinction along the line of sight and is therefore a suitable object for reddening studies. In A14, we used the available UV-NIR spectroscopic time series (Pereira et al. 2013; Mazzali et al. 2014) and the NIR light curves (Matheson et al. 2012) to derive the extinction law of SN 2014J. In this work, we extend this template with UV photometry (Brown et al. 2012) and optical photometry at 25 to $40 \mathrm{~d}$ past $B$-band maximum.

The light curves in the UV F225W, F275W, F336W and the optical $B V R i$ bands are first constructed by synthesizing the spectra from Mazzali et al. (2014) and Pereira et al. (2013). A light curve for each filter is then obtained by fitting smoothed splines using SNOOPY (Burns et al. 2011). For the redder optical $R$ and $i$ bands, the sparse temporal coverage after $20 \mathrm{~d}$ past $B$-maximum does not allow an accurate spline fit of the second bump. We therefore also use the data from Munari et al. (2013) to constrain the spline fit at $>25 \mathrm{~d}$ past $B$-max. For the Swift/UVOT uvm 2 filter and the NIR JHKs we fit spline models to the measured light curves from Brown et al. (2012) and Matheson et al. (2012), respectively. The light curves are then used to calculate the $(X-V)_{0}$ colours shown in Fig. 5 .

As explained in Section 4, we also need a template of the spectral energy density of the unreddened object in order to be able to calculate the expected extinction for each passband for a given extinction model. In A14 the extinction for a given set of reddening law parameters was first calculated for all available spectra of SN 2011fe and the extinction for any given epoch was then obtained using spline interpolations. Similar to the light-curve spline model, this approach is adequate for the UV, but due to the sparse spectral coverage, it will cause the model to deviate from the light curve for the redder optical bands and NIR at $>25 \mathrm{~d}$ past maximum. In A14 this did not affect the final results due to the high extinction of SN 2014J and the conservative intrinsic colour dispersions adopted in the analysis.

In this work, we have developed a more accurate SED template by first resampling the spectral time series used in A14 to a resolution of $10 \AA$, which are shown as the red spectra in Fig. B1. These were then used to calculate the missing wavelength and temporal elements through linear interpolation (shown in grey in Fig. B1), and finally the full matrix is mangled to match the light curves described above. The mangling was carried out by first calculating the ratio between the light-curve fluxes and the corresponding synthetic SED fluxes. A function was then setup for each phase where the calculated ratios were adopted for the effective wavelengths of the filters. For all wavelengths shorter (longer) than the bluest (reddest) point, the function was assumed to take the same value as in the extreme points, while we interpolate the function value for all intermediate wavelengths, using the calculated ratios. The SED for the given phase was then multiplied by the function. The difference between the synthetic colours calculated from the mangled SED and the spline colour model was always found to be within $<0.05$ mag for all epochs, and often even smaller. In Fig. B2 these differences are shown for six different colours. Since these differences are within the adopted intrinsic colour uncertainties they will not have any significant impact on the fitted reddening laws. 


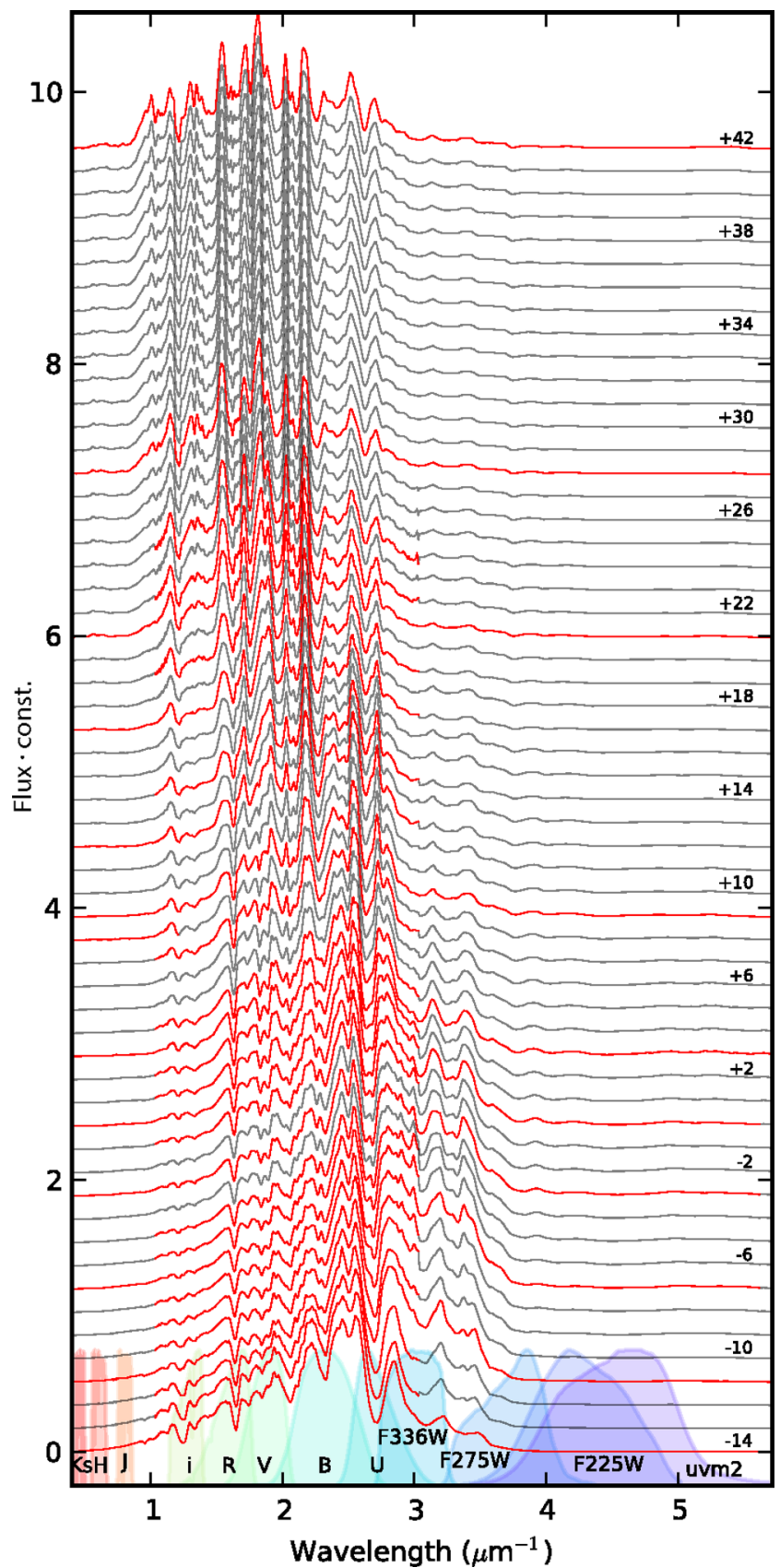

Figure B1. The spectral template constructed from measured spectra of SN 2011fe. Sections based on measured data are shown in red, while the interpolated sections, warped to the light curves are shown in grey. The filters used for the warping are shown at the bottom of the figure.

\section{APPENDIX C: A MODEL FOR THE UV OF SN 2011 by}

The SNe 2011by and 2011fe have been found to be remarkably similar in the optical, both around maximum and at nebular phases, while showing significant discrepancies in the UV (Foley \& Kirshner 2013; Graham et al. 2015a). In Fig. 14, the ratio between the spectra are shown at maximum to illustrate this. Unfortunately, SN 2011 by does not have the wavelength and temporal coverage that SN $2011 \mathrm{fe}$ has, but we can create a crude template for SN 2011by based on the assumption that it is indeed identical to SN $2011 \mathrm{fe}$ with the exception of the UV wavelengths.

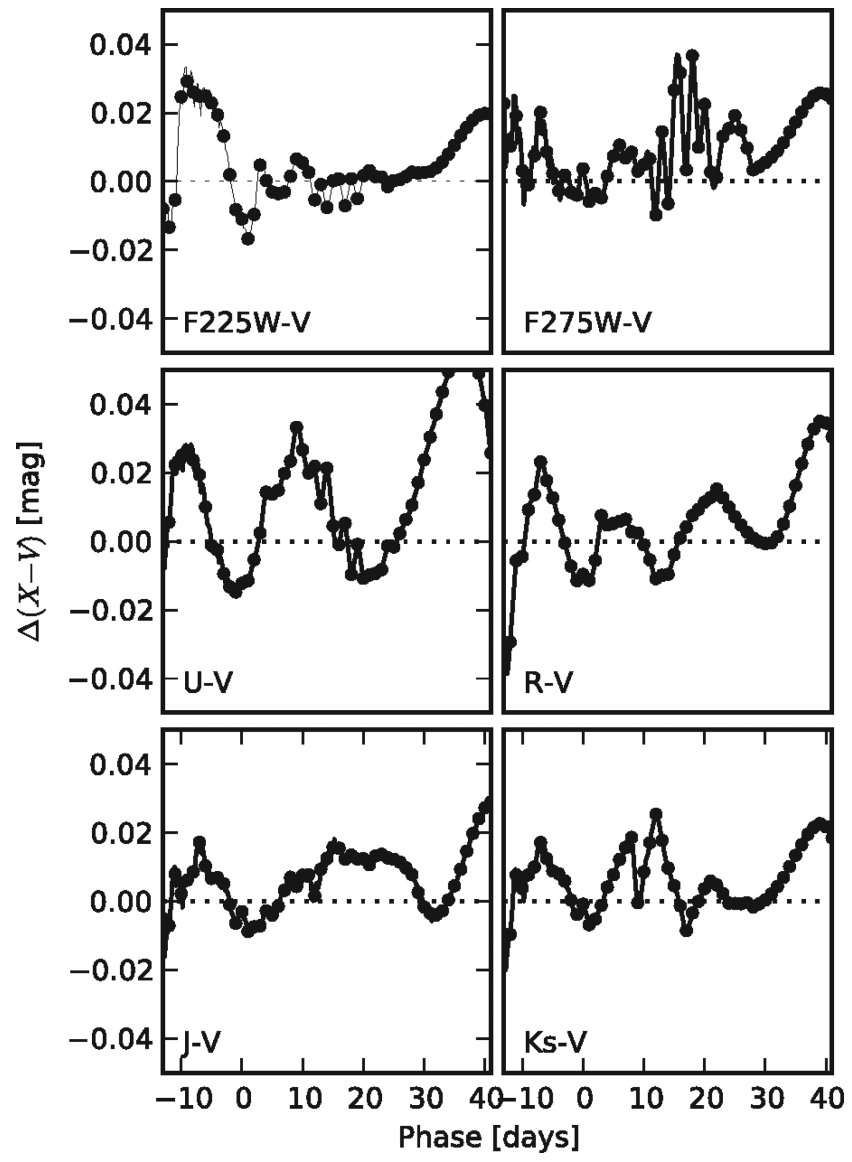

Figure B2. Examples of the difference between synthetic colours calculated from the SED template, and the corresponding colours from the light-curve spline templates.

In Fig. 14 the solid green line is showing the function

$f\left(x^{\prime}\right)=c\left[\frac{x^{\prime}}{\left(x^{\prime 4}+1\right)^{1 / 4}}-1\right]+1 \quad$ with $\quad x^{\prime}=\frac{x-x_{0}}{\alpha}$,

where $x$ is the wavelength given in microns. In the figure, the function is plotted for the parameters $x_{0}=0.2495, \alpha=0.0360$ and $c=0.3359$.

SN 2011by was observed by Swift in the UV (M13) and in the optical (Silverman et al. 2013b), and the colour excesses of these measurements with respect to SN 2011fe are shown in Fig. C1. Here, we note that $E(u v m 2-V)$ is showing a similar time-evolution as was seen for SN 2012cg in the upper-left panel of Fig. 6.

Under the assumption that SNe 2011by and $2011 \mathrm{fe}$ are twins at wavelengths $\lambda>3000 \AA$ we can create an approximate template for SN 2011 by by multiplying the SED of SN 2011fe, presented in Appendix B and shown in Fig. B1, by equation (C1). Further, if we fix the parameters $x_{0}$ and $\alpha$ to the values given above, we can fit $c$ for each epoch with $u v m 2$ observations by forcing the synthetically calculated $A_{u v m 2}$, based on the SED to match the observed $E($ uvm $2-V)$ in Fig. C1 (again, assuming that $\left.V^{2011 \mathrm{fe}}=V^{2011 \mathrm{by}}\right)$. The fitted values of $c$ are shown in Fig. $\mathrm{C} 2$ and the synthetic values of $A_{\text {uvm } 2}$ that these values correspond to are been plotted as black dots in Fig. C1.

Further, we parametrize $c(p)$ with a Gaussian,

$c(p)=\frac{a}{\sqrt{2 \pi} \sigma} \exp \left[-\frac{1}{2}\left(\frac{p-p_{0}}{\sigma}\right)^{2}\right]+b$, 


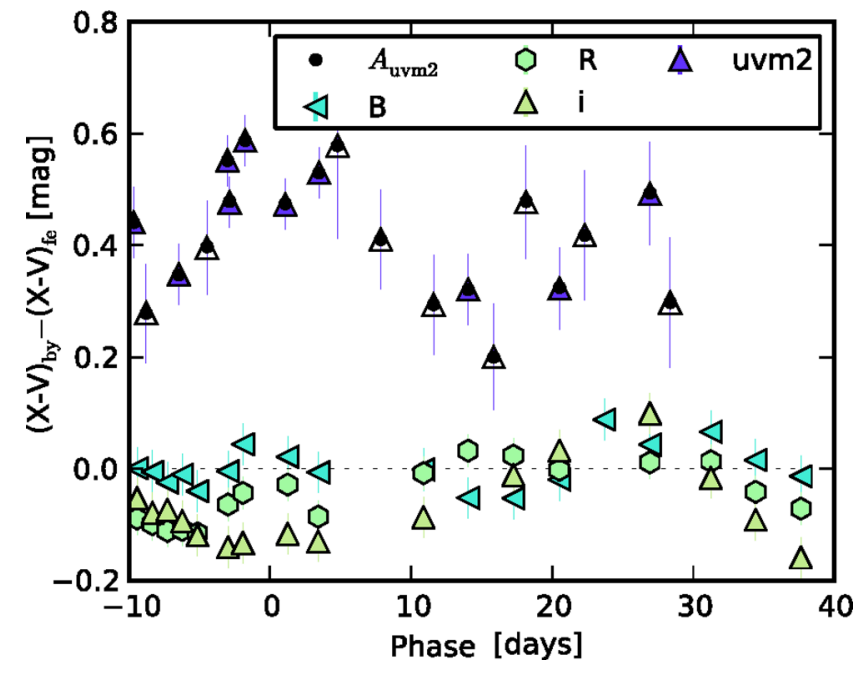

Figure C1. The difference in colour between SNe 2011by and 2011fe, where the ground-based photometry has been adopted from Silverman et al. (2013b). Observations for which the colour was obtained from measured $V$-band data are shown with filled symbols, while values obtained from a $V$-band template are shown with open symbols. The colour difference between the $\mathrm{SNe}$ can also be calculated synthetically based on equation (C1) under the assumption that this function describes the flux ratio of the two objects, for all epochs, after adjusting for the difference in distance. This is motivated by the observation that these $\mathrm{SNe}$ appear to be twins at all wavelengths except for the UV. The parameter $c$ in the equation was fitted by matching the synthetic and measured $u v m 2-V$ for each epoch, where the former is shown by the black points for the best fitted values.

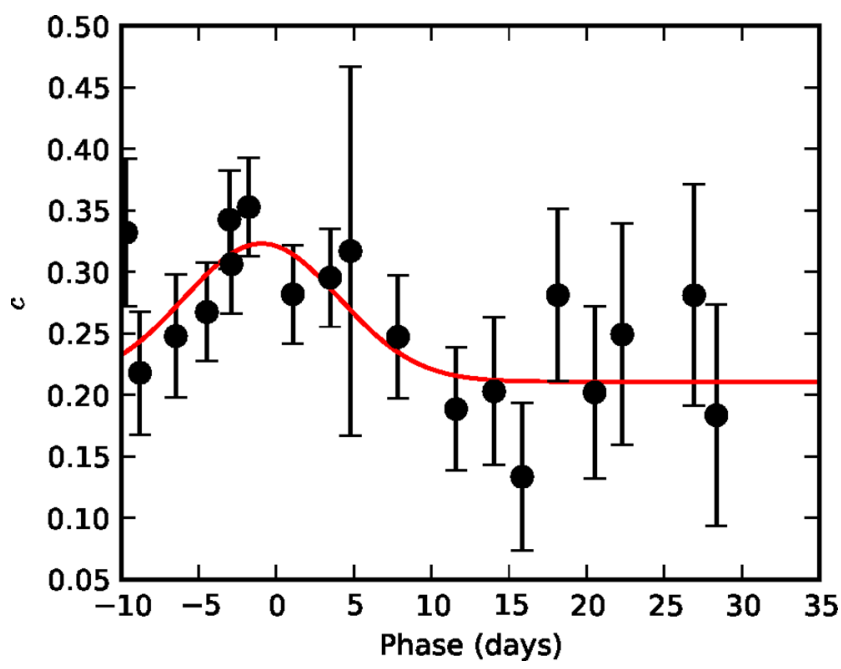

Figure C2. The fitted values of parameter $c$ in after the SED of SN $2011 \mathrm{fe}$ has been fitted together with equation (C1) to match SN 2011by uvm2 photometry. The solid line is showing a Gaussian fit.

as shown by the solid line in Fig. C2, with $p_{0}=-0.939, a=1.406$, $b=0.211$ and $\sigma=4.992$.

To summarize, we create a crude light curve and SED template of SN 2011 by by using the template of SN 2011 fe from Section B and multiply it with equation $(\mathrm{C} 1)$, where the parameters, including the time-dependent $c$, are given above.

\section{APPENDIX D: RED TAILS OF THE HST FILTERS}

The HST filter transmission used in this work, shown in the upper panel of Fig. D1, suffer from red tails. That is, part of the flux observed for an object in these filters originates from wavelengths that are redder than the range where most of the transmission lies. As mentioned in A14, the red tails are relatively small for the WFC3/UVIS filters, e.g. the transmission of $F 225 \mathrm{~W}$ is roughly one part in 100000 at $5000 \AA$ compared to the peak transmission at $\sim 2250 \AA$. Despite this, the red tails will have implications when reddened SNe Ia are studied. For example, from spectrophotometry of SN 2011fe at maximum (thin blue line in the middle panel of Fig. D1), we conclude that $\sim 0.5$ per cent of the flux (dashed blue line) in the $F 225 W$ filter will originate from wavelengths $>4000 \AA$. However, extinction will suppress the UV-flux significantly and after applying an F99 reddening law with $E(B-V)=1.4, R_{V}=1.4$, $\sim 60$ per cent of the $F 225 \mathrm{~W}$ flux (thick blue line) comes from photons with $\lambda>4000 \AA$.

Another way to illustrate the effect of the red tails is shown in the lower panel of Fig. D1, where the solid lines show the effective median wavelengths for an observation of SN $2011 \mathrm{fe}$ at maximum through the WFC filters with various amounts of reddening applied. The effective median wavelength is defined such that 50 percent of the light originates from redder and bluer wavelengths, respectively. Further, the wavelength range defined by the points where 10 percent and 90 percent or the light comes from bluer wavelengths (i.e. the region that includes 80 percent of the light) is shown for increasing reddening. From the figure we conclude that, $F 275 W$ and $F 336 W$ are less plagued by red tails than $F 225 W$, where the latter, in fact, will be probing redder effective wavelengths of the SN spectrum than the two former filters for $E(B-V) \gtrsim 1 \mathrm{mag}$.

The fact that we are comparing different effective wavelengths when calculating the colour excess between a reddened and unreddened source is true for all broad-band observations. However, fitting extinction properties based on filters with red tails that span a wavelength range where the SED drops rapidly, also puts demands on knowing the passband throughput accurately even when the transmission drops several orders of magnitude as for the filters in Fig. D1.

The expected WFC3/UVIS passband red tails were tested (Brown 2011) in the final round of thermal vacuum tests of WFC3 prior to launch. They studied the filter throughputs in four wavelength bins centred on 450,600, 750 and $900 \mathrm{~nm}$ and found deviations of up to $\sim 40$ per cent between the measured and expected transmission. If the deviations are taken as an estimate of the throughput uncertainty at these wavelengths, we can evaluate its impact on the extinction analysis.

We carried out this test by first applying an F99 reddening law with $E(B-V)=1.4, R_{V}=1.4$ to the SED of SN $2011 \mathrm{fe}$ described in Section 4.1. The impact on the calculated extinction for the two bands $F 225 W$ and $F 275 W$ (the test of $F 336 W$ was not carried out due to limitations in the setup as described in Brown 2011) can then be calculated. We do this by comparing synthetic magnitudes from the original SYNPHOT throughputs, to magnitudes obtained after the filters have been multiplied by a spline function derived from the deviations for the four wavelength bins mentioned above. The impact on the calculated extinction was found to always be $<0.05$ mag. Further, the worst case scenario can be estimated by letting all the measured deviations from Brown (2011) maximize the amount of red light entering the filter. The result on the extinction from this was $<0.10$ mag for $t<20 \mathrm{~d}$ with respect to $B$ max and $0.15-0.20$ mag 


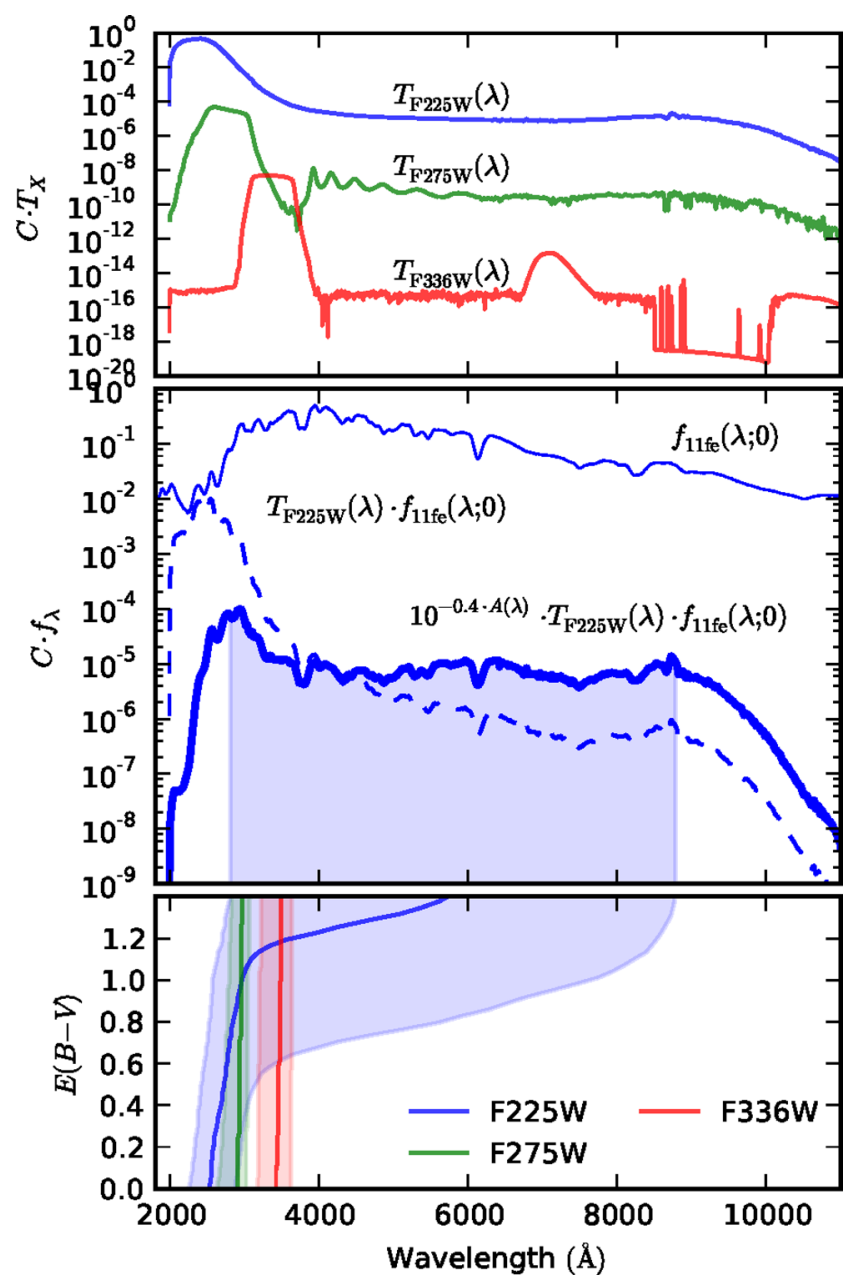

Figure D1. The upper panel shows the filter transmissions, $T_{X}(\lambda)$, of the WFC3/UVIS passbands, multiplied with an arbitrary normalization, $C$. The thin blue line in the middle panel shows the spectrum of SN 2011fe at $B$ band maximum, while the dashed blue line shows the same spectrum after it has been multiplied by the $F 225 \mathrm{~W}$ throughput. The thick blue line shows the same spectrum after it has been reddened with an F99 extinction law with $E(B-V)=1.4 \mathrm{mag}$ and $R_{V}=1.4$, similar to what we observe for SN 2014J. All three spectra have arbitrary normalization, $C$. In the plot, we have also marked the wavelength region from which 80 per cent of the light originates from, defined such that 10 percent of light comes from bluer wavelengths, and 10 per cent comes from redder wavelengths. The lower panel shows the median wavelength, i.e. where 50 per cent of the light originates from redder and bluer wavelengths, for three passbands for SN 2011fe at $B$ maximum, reddened with an F99 extinction law $\left(R_{V}=1.4\right)$ with increasing extinction. The 80 per cent region is shown in the same manner as for the middle panel.

for $t=20-40 \mathrm{~d}$, which is still within the dispersion that we have adopted for the intrinsic UV colours.

It is also possible to investigate the accuracy of the full HST/WFC3/UVIS SYNPHOT throughput by comparing spectrophotometry of objects, with known spectra, to WFC3/UVIS photometry. This is in fact the most direct test we can carry out of our method for fitting extinction laws since this is using the very same procedure. In Fig. D2, we have plotted stars from CALSPEC ${ }^{8}$ with the corresponding UVIS-photometry using the same analysis path as in Section 2.1 with a 0.4 arcsec radius. The agreement is in gen-

${ }^{8}$ http://www.stsci.edu/hst/observatory/crds/calspec.html

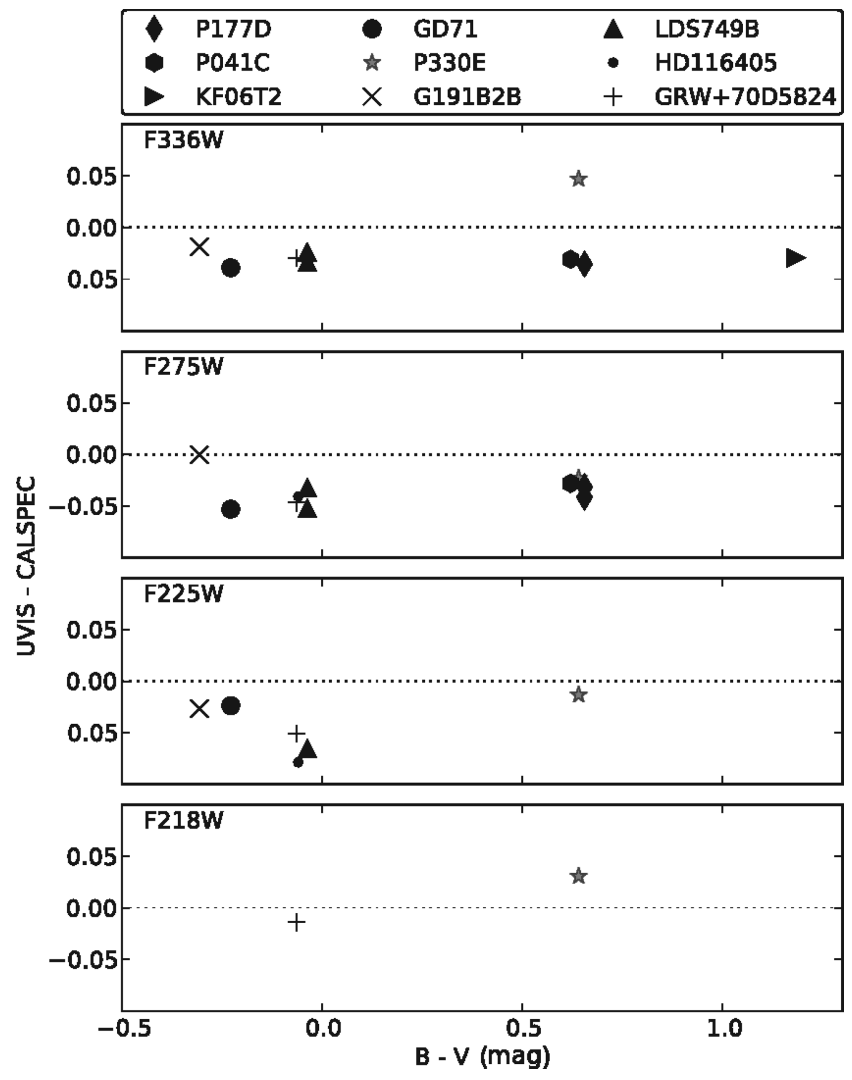

Figure D2. Comparison between spectrophotometry of CALSPEC stars using the WFC3/UVIS filter throughputs, with the corresponding WFC3/UVIS photometry against the synthetically calculated $B-V$ colour. The star P330E was used for calibrating WFC3/UVIS.

eral more than sufficient for the purpose of this analysis, but it is unfortunate that data are not available for redder stars for which the impact of the red tail can be expected to be more significant. The star P330E should be discarded from the comparison since this star has been used for calibrating the WFC3/UVIS system.

However, we can carry out the same test for the reddened $\mathrm{SNe}$ Ia that have been observed with both STIS and WFC3/UVIS. In Table D1, we present spectrophotometry at maximum for SNe $2012 \mathrm{cg}$ and 2014J, using the transmissions of the WFC3/UVIS filters, compared to the corresponding WFC3/UVIS photometry. For

Table D1. Spectrophotometry of STIS spectra at maximum of SNe 2012cg and 2014J at maximum using the filter transmissions shown in Fig. D1 compared to WFC3/UVIS photometry of the same objects. For SN 2012cg the observations were obtained in consecutive HST orbits, while the data for SN 2014J was taken $1 \mathrm{~d}$ apart. For this SN, we have calculated the magnitude difference synthetically from the SN 2011 fe spectra described in Appendix B. Since the colour evolution is slow between the two observations, any discrepancy in the filter transmissions will be of second order for this correction.

\begin{tabular}{lllc}
\hline SN & Filter & STIS & WFC \\
\hline $2012 \mathrm{cg}$ & $F 225 W$ & 15.89 & $15.98(0.05)$ \\
& $F 275 W$ & 13.67 & $13.61(0.05)$ \\
$2014 \mathrm{~J}$ & $F 336 W$ & 11.85 & $11.74(0.06)$ \\
& $F 225 W$ & 18.43 & $18.50(0.04)$ \\
& $F 275 W$ & 16.55 & $16.55(0.04)$ \\
& $F 336 W$ & 13.15 & $13.02(0.04)$ \\
\hline
\end{tabular}


$2014 \mathrm{~J}$, the spectrum and photometry were obtained on consecutive days, and the magnitudes based on the former were correct to correspond to the WFC3/UVIS epoch using the SN 2011fe template. These corrections are $<0.1 \mathrm{mag}$.

For these two $\mathrm{SNe}$, the discrepancies between two instruments are within $0.1 \mathrm{mag}$, including $F 225 \mathrm{~W}$. This is the filter with the most significant red tails, and the photon wavelength distribution for $F 225 \mathrm{~W}$ is significantly different for two SNe. The consistency between spectrophotometry and WFC3/UVIS photometry for two SNe that define the end points of the reddening range in our study is showing that any errors of the filter transmissions will not affect the conclusions drawn in the paper.

Further, the fact that we also observe a discrepancy of $\sim 0.1$ mag for the $F 336 W$ filter, which has minor red tails, suggests that the discrepancies we observe for $F 225 \mathrm{~W}$ may not necessarily originate from uncertainties of these.

${ }^{1}$ Oskar Klein Centre, Physics Department, Stockholm University, SE-106 91 Stockholm, Sweden

${ }^{2}$ George P. and Cynthia Woods Mitchell Institute for Fundamental Physics \& Astronomy, Texas A. \& M. University, Department of Physics and Astronomy, 4242 TAMU, College Station, TX 77843, USA

${ }^{3}$ Cahill Center for Astrophysics, California Institute of Technology, Pasadena, CA 91125, USA

${ }^{4}$ Carnegie Observatories, Las Campanas Observatory, Colina El Pino, Casilla 601, La Serena, Chile

${ }^{5}$ Institute of Theoretical Astrophysics, University of Oslo, PO Box 1029, Blindern, N-0315 Oslo, Norway

${ }^{6}$ INAF - Osservatorio Astronomico di Padova, vicolo dell'Osservatorio 5, I-35122 Padova, Italy

${ }^{7}$ Dark Cosmology Centre, Niels Bohr Institute, University of Copenhagen, Juliane Maries Vej 30, DK-2100 Copenhagen O, Denmark

${ }^{8}$ Instituto de Astrofísica de Andalucía (IAA-CSIC), Glorieta de la Astronomía s/n, E-18008 Granada, Spain

${ }^{9}$ Unidad Asociada Grupo Ciencias Planetarias UPV/EHU-IAA/CSIC, Departamento de Física Aplicada I, E.T.S., Ingeniería, Universidad del País Vasco UPV/EHU, Alameda de Urquijo s/n, E-48013 Bilbao, Spain
${ }^{10}$ INAF- Osservatorio Astronomico di Roma via Frascati 33, I-00040 Monte Porzio Catone, Roma, Italy

${ }^{11}$ Las Cumbres Observatory Global Telescope Network, 6740 Cortona Dr, Suite 102, Goleta, CA 93117, USA

${ }^{12}$ Department of Physics, University of California, Santa Barbara, Broida Hall, Mail Code 9530, Santa Barbara, CA 93106-9530, USA

${ }^{13}$ Department of Physics and Astronomy, Aarhus University, Ny Munkegade 120, DK-8000 Aarhus C, Denmark

${ }^{14}$ Astrophysics Research Centre, School of Mathematics and Physics, Queen's University Belfast, Belfast BT7 1NN, UK

${ }^{15}$ Observatories of the Carnegie Institution for Science, 813 Santa Barbara Street, Pasadena, CA 91101, USA

${ }^{16}$ Benoziyo Center for Astrophysics, Faculty of Physics, Weizmann Institute of Science, 76100 Rehovot, Israel

${ }^{17}$ Oskar Klein Centre, Astronomy Department, Stockholm University, SE-106 91 Stockholm, Sweden

${ }^{18}$ Finnish Centre for Astronomy with ESO (FINCA), University of Turku, Väisäläntie 20, FI-21500 Piikkiö, Finland

${ }^{19}$ Department of Astronomy, University of California, Berkeley, CA 94720 3411, USA

${ }^{20}$ Computational Cosmology Center, Lawrence Berkeley National Laboratory, 1 Cyclotron Road, Berkeley, CA 94720, USA

${ }^{21}$ CENTRA - Centro Multidisciplinar de Astrofísica, Instituto Superior Técnico, Av. Rovisco Pais 1, P-1049-001 Lisbon, Portugal

${ }^{22}$ School of Physics and Astronomy, University of Southampton, Southampton SO17 1BJ, UK

${ }^{23}$ Tuorla Observatory, Department of Physics and Astronomy, University of Turku, Väisäläntie 20, FI-21500 Piikkiö, Finland

${ }^{24}$ Department of Astronomy, Faculty of Mathematics, University of Belgrade, Studentski trg 16, 11000 Belgrade, Serbia

${ }^{25}$ Department of Earth and Space Sciences, Chalmers University of Technology, Onsala Space Observatory, SE-439-92 Onsala, Sweden

${ }^{26}$ ASTRON, Postbus 2, NL-7990 AA Dwingeloo, the Netherlands

${ }^{27}$ Kapteyn Astronomical Institute, Postbus 800, NL-9700 AV Groningen, the Netherlands

This paper has been typeset from a $\mathrm{T}_{\mathrm{E}} \mathrm{X} / \mathrm{L} \mathrm{T} \mathrm{E} \mathrm{X}$ file prepared by the author. 